The Properties of Hydrogen and Helium Under Extreme Conditions

J. M. McMahon, M. A. Morales, C. Pierleoni, D. M. Ceperley

December 5, 2011

Reviews of Modern Physics 
This document was prepared as an account of work sponsored by an agency of the United States government. Neither the United States government nor Lawrence Livermore National Security, LLC, nor any of their employees makes any warranty, expressed or implied, or assumes any legal liability or responsibility for the accuracy, completeness, or usefulness of any information, apparatus, product, or process disclosed, or represents that its use would not infringe privately owned rights. Reference herein to any specific commercial product, process, or service by trade name, trademark, manufacturer, or otherwise does not necessarily constitute or imply its endorsement, recommendation, or favoring by the United States government or Lawrence Livermore National Security, LLC. The views and opinions of authors expressed herein do not necessarily state or reflect those of the United States government or Lawrence Livermore National Security, LLC, and shall not be used for advertising or product endorsement purposes. 


\title{
The Properties of Hydrogen and Helium Under Extreme Conditions
}

\author{
Jeffrey M. McMahon, ${ }^{1}$ Miguel A. Morales, ${ }^{2}$ Carlo Pierleoni, ${ }^{3}$ and David M. Ceperley ${ }^{1,4, *}$ \\ ${ }^{1}$ Department of Physics, University of Illinois at Urbana-Champaign, Urbana, Illinois 61801, USA \\ ${ }^{2}$ Lawrence Livermore National Laboratory, Livermore, California 94550, USA \\ ${ }^{3}$ Department of Physics, Università of L'Aquila and CNISM UdR L'Aquila, Via Vetoio 10, I-67010 L'Aquila, \\ Italy \\ ${ }^{4}$ NCSA, University of Illinois at Urbana-Champaign, Urbana, Illinois 61801, USA
}

(Dated: May 17, 2012)

\begin{abstract}
Hydrogen and helium are the most abundant elements in the universe and, in principle, are the simplest elements. Nonetheless, they display remarkable properties under pressure that have fascinated theoreticians and experimentalists for over a century. Recent advances in computational methods have made it possible to elucidate many of these properties. We review some of the computational methods that have been applied to dense hydrogen and helium in recent years, mainly those that perform a simulation directly from the physical picture of electrons and ions; primarily, those based on density functional theory and quantum Monte Carlo methods. We then discuss the predictions from such methods as applied to the phase diagram of hydrogen, including the solid and fluid phases, with particular focus on the crystal structures, the liquidliquid transition and comparison of the results with experimental shock-wave data. We then discuss predictions of ordered quantum states, including a possible low-temperature fluid and high-temperature superconductivity in the atomic state. We also briefly discuss pure helium, and then focus on hydrogen-helium mixtures, with particular focus on properties of relevance to planetary science.
\end{abstract}

PACS numbers: 62.50.-p, 67.80.F-, 81.30.-t, 67.80.-s, 61.20.Ja

\section{Contents}

Glossary

I. Introduction

II. Predicting Properties of Matter under Extreme Conditions

A. The Formalism of Imaginary-time Path Integrals

B. The Born-Oppenheimer Approximation

C. Path Integral Monte Carlo

D. QMC-based First-Principles simulations

1. Ground-state Quantum Monte Carlo methods

2. Coupled Electron-Ion Monte Carlo (CEIMC)

E. DFT-based First Principles simulations

1. Density Functional Theory

2. Treatment of proton zero point motion

3. Born-Oppenheimer Molecular Dynamics

4. Car-Parrinello Molecular Dynamics

5. Path Integral Molecular Dynamics

F. Size Effects

G. Other Theoretical Methods

1. One Component and Screened Coulomb Plasma Models

2. Semi-empirical Methods and Chemical Models

H. Comparison of Simulation Methods

III. Experimental Methods

A. Dynamical Compression

B. Static Compression

C. Coupling Static and Dynamic Compressions

IV. Hydrogen under Extreme Conditions

A. Solid Phases
1. Solid Molecular Hydrogen at Low-temperature

2. Solid Molecular Hydrogen at High-temperature

3. Additional Solid Molecular Phases

4. Melting curve of the molecular crystal

5. The Metallization of solid Molecular Hydrogen

6. Atomic crystal phases

B. The Normal Fluid Phases

1. The Equation of State and Principal Hugoniot of Hydrogen

2. Liquid-Liquid Phase Transition

3. Optical and Transport Properties

C. Quantum Phases of High Pressure Hydrogen

1. Superconductivity in Solid Hydrogen

2. A Quantum Fluid?

V. Helium and Hydrogen-Helium Mixtures

A. Helium

1. Equilibrium Properties: Equation of State and Structure

2. Dynamic Properties: Optical and Transport Properties

B. Phase separation of $\mathrm{H}$ and $\mathrm{He}$

21

24

25

26

27

29

30

30

34

36

37

37

38

39

40

40

41

42

VI. Conclusions and Outlook

45

Acknowledgments

46

References

47

\section{Glossary}

$B$ Rotational constant of a molecule. 22

$T_{c}$ Superconducting critical temperature. 37, 38

$T_{m}$ Melting temperature. 38 
$\lambda$ Attractive electron-phonon-induced interaction. 37

$\langle\omega\rangle$ Average phonon frequency. 37

$\mu^{*}$ Renormalized Coulomb repulsion. 37

$\mathbf{D}_{2}$ Molecular deuterium. 20, 22-24

$\mathbf{H}_{2}$ Molecular hydrogen. 20, 22, 23

AIRSS $A b$ initio random structure searching. 23, 25, 26

bcc body-centered cubic. 29

BO Born-Oppenheimer Molecular Dynamics. 16

BO Born-Oppenheimer. 9, 16, 23

BSP Broken symmetry phase of molecular hydrogen, also known as Phase II. 21, 22

CEIMC Coupled Electron-Ion Monte Carlo. 30

CPMD Car-Parrinello Molecular Dynamics. 15, 16, 26, 29

DAC Diamond anvil cell. 3, 20, 21, 25-28, 45

DF Density functional. 14, 18, 24, 26

DFT Density functional theory. 4, 14, 15, 18, 23, 24, 26-28, 37

EOS Equation of state. 2, 3, 20, 30

EQ Electric-quadrupole. 22

fcc Face-centered cubic. 22, 29

FPMD First-Principles Molecular Dynamics. 27

hcp Hexagonal close-packed. 22-27, 29

HK Hohenberg-Kohn. 13

HSE Heyd-Scuseria-Ernzerhof. 14

ICF Inertial confinement fusion. 4

IM Insulator-to-metal (transition). 27, 28

IR Infrared. 20, 24-26

KS Kohn-Sham. 13, 14

LDA Local density approximation. 22

LLT Liquid-liquid (phase) transition. 30, 45

OCP One-component plasma. 38
PIMC Path-integral Monte Carlo. 22, 24

PIMD Path-integral molecular dynamics. 24

PPT Plasma phase transition. 3

PSO Particle-swarm optimization. 26, 29

QMC Quantum Monte Carlo. 4, 15, 18, 19, 23, 24, 28, $29,38,39,46$

SCDFT Density functional theory of superconducting state. 37

SCP Screened Coulomb Plasma. 29, 30

vdW van der Waals. 14, 24

ZPE Zero-point energy. 15, 23-25, 29, 39

ZPM Zero-point motion. 15, 23-27, 29, 39, 46

\section{INTRODUCTION}

Hydrogen, being the first element, is correspondingly referred to as the most simple element. The equations of quantum mechanics can be solved exactly for a single atom, and thus is the mainstay of elementary Quantum Mechanics textbooks. The Helium atom, with only two electrons, is no longer analytically solvable, but still simple relative to heavier atoms. However, despite their atomic simplicities, bulk hydrogen and helium are surprisingly complex. In this review, we describe what has been learned from experiments, theory, and computation about bulk hydrogen and helium under a variety of thermodynamic conditions of temperature and pressure, in particular, extreme conditions hard to achieve in the laboratory.

The primary motivation to study dense hydrogen and helium under extreme conditions comes from the fact that they are the most abundant elements in the universe. For example, they form from $70 \%-95 \%$ of the mass of Jupiter and Saturn in our own solar system, as well as being the principle components of a large number of recently discovered exo-planets (Baraffe et al., 2010). What is needed to model the planets is the equilibrium equation of state (EOS), the pressure as a function of temperature, density, and composition. Errors in the EOS lead to unreliable estimates of what is inside a planet, whether there are elements other than $\mathrm{H}$ and $\mathrm{He}$, the past history of the planets and the process of planetary formation (Fortney, 2004; Fortney and Nettelmann, 2010). A long-standing puzzle is to understand why Saturn is 50\% more luminous than existing models. Additional energy sources could come from $\mathrm{H}-\mathrm{He}$ demixing (Fortney and Hubbard, 2003) as proposed by Smoluchowski, 1967. In addition, the abundance in Saturn's atmosphere shows depletion of He. Working backward from astronomical observations 


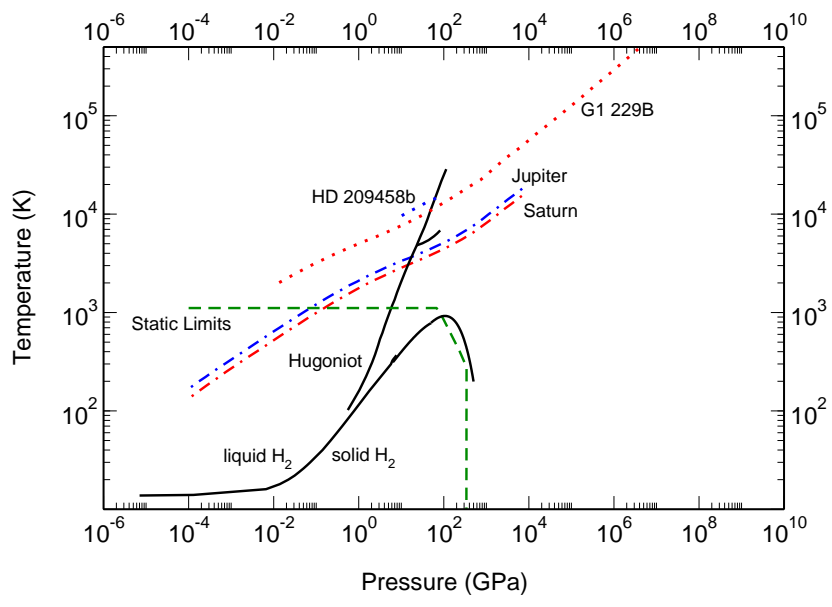

FIG. 1 (Color online) The phase diagram (temperature vs pressure) for hydrogen. The principal Hugoniot of hydrogen (the densities and pressures that can be reached by shocking solid hydrogen initially at 1 Bar) is shown as a solid line, the secondary Hugoniot (i.e. points reached with a double shock) is also shown branching downward from the principal Hugoniot. The isentropes of three giant planets (Jupiter, Saturn, and HD 209458b) and a representative brown dwarf (G1 229B) are shown as dashed lines; the estimated melting temperatures of $\mathrm{H}_{2}$ is shown as a dashed-dot line. Static diamond anvil experiments are able to access temperatures less than roughly $1100 \mathrm{~K}$ and pressures less than $300 \mathrm{GPa}$ as delimited by the green dashed-line.

is a very indirect and uncertain way of learning about the phase diagram of hydrogen-helium mixtures. It is estimated that the EOS needs to be accurate to $~ 1 \%$ to answer fundamental questions about the composition and formation of Jovian planets and to make reliable models of exo-planets (Stevenson, 2010).

Figure 1 shows the part of the phase diagram accessible to experiment and the conditions needed to understand the planets: the isentropes of some of the wellcharacterized giant planets, Jupiter and Saturn (Saumon and Guillot, 2004), one of the most well-characterized exoplanets, HD 209458b (Guillot and Showman, 2002; Nellis, 2006b), and a representative brown dwarf, G1 229B (Burrows et al., 2001), are shown.

The planetary isentropes lie outside the realm of static diamond anvil cell (DAC) experiments, which while they reliably can extend to pressures of $\sim 320 \mathrm{GPa}$ (Goncharov et al., 2001; Loubeyre et al., 2002), can only do so at relatively low temperatures, such as $1115 \mathrm{~K}$ at $73 \mathrm{GPa}$ (Gregoryanz et al., 2003). The experimental range of static measurements is depicted in Fig. 1. Dynamic shock compression experiments (Nellis, 2006b), on the other hand, can access similar temperature and pressure conditions as well as those much more extreme. However, the conditions do not overlap completely and these experiments have great uncertainties, likely far greater than the aforementioned $\sim 1 \%$ accuracy desired (Stevenson, 2010).
Another important motivation for studying hydrogen and helium is to understand and predict the properties of the simplest elements of the periodic table. Much is still unknown about hydrogen. The longest outstanding issue concerns an insulator-to-metal transition: the observation of metallic hydrogen has often been called the "holy grail" of high pressure research. Early predictions suggested that hydrogen would become a simple atomic metal at a pressure of $25 \mathrm{GPa}$ (Wigner and Huntington, 1935). As experimental pressures have steadily increased to beyond $300 \mathrm{GPa}$, this transition has not yet been seen, at least not at room temperature or below. Early predictions were based on the assumption that metallic hydrogen would be a simple metal with nondirectional metallic bonding. However, recent work suggests that this is not the case and hydrogen will go through a sequence of phase transitions, first in the molecular phase and then in the atomic phase. One can either view this transition as a change from molecules to atoms or a change from insulator to metal. Both transitions are plausible and according to Wigner's scaling argument inevitable, but do the transitions happen together or separately?

Figure 2 shows on an expanded scale the estimated physical regimes of hydrogen as it transforms from a molecular crystal at low density to an atomic crystal at high density, and, upon increasing temperature, as it first melts and then transforms into a plasma state.

While metallic hydrogen has not yet been observed in the low temperature solid, metallization has been achieved in the fluid state in the range $100-200 \mathrm{GPa}$ and $2000-3000 \mathrm{~K}$, as discuss later in this review. But important questions in characterizing the transition from insulating molecular hydrogen to metallic atomic hydrogen remain: is it a first-order transition or a simple crossover? Landau and Zeldovich, 1943 speculated that the insulator-metal change at increasing density in liquid mercury would be a genuine phase transition. Since then, there has been recurring controversy concerning whether this transition, the so-called "plasma phase transition" (PPT), a second liquid-liquid transition(LLT) on the phase diagram, exists, and what its relation to the molecular dissociation process is. See (Redmer and Holst, 2010) for a recent overview. If the PPT exists at high enough temperatures, then there could be a surface inside the giant planets separating a conducting core from an insulating mantle. What is clear is that a number of different physical phenomena come together in the middle of the phase diagram: the metal-insulator transition and/or atomic-molecular transitions caused by a combination of temperature and pressure. Even the zero point motion of the protons can play an important role.

As temperature is lowered, the liquid will freeze. It has been suggested (Mon et al., 1980) that because of electronic screening, the effective proton-proton interaction is much reduced so that the liquid could remain stable at low temperatures. In fact, a large depression of the melting point temperature is seen in other alkali metals such as sodium and lithium. But if hydrogen is a liquid at suf- 


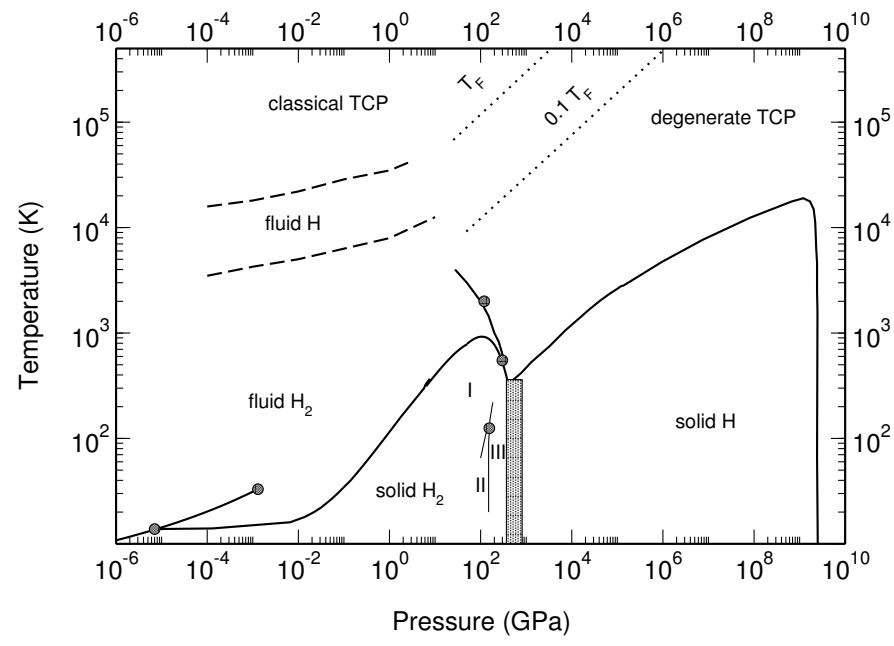

FIG. 2 Hydrogen Phase diagram. Solid lines show the boundaries between the gas, liquid and solid phases. The solid circles show location of critical and triple points. The dashed lines on the left estimate when fluid hydrogen changes from $\mathrm{H}_{2}$ to fluid $\mathrm{H}$ and then to a classical two component plasma (TCP). The dotted lines on the above $10^{6}$ bar estimate the temperature when the electrons become degenerate: the noninteracting fermi energy $E_{F}$ and $0.1 E_{F}$. Also shown are three phases (I,II,III) of solid $\mathrm{H}_{2}$ which occur as the molecules become more oriented. How precisely hydrogens change from solid $\mathrm{H}_{2}$ to solid $\mathrm{H}$ is not established so it is shown as a grey box. The line going vertically away from the grey box shows the separation between the mostly insulating molecular fluid and the mostly conducting atomic fluid; the first order liquidliquid transition ends at a critical point; what is shown at higher temperatures is a crossover. The almost vertical transition line at the extreme right of the diagram indicates the quantum melting of the protons lattice under compression.

ficiently low temperatures, the effects of quantum statistics of the light protons could be important and could lead to very interesting phases such as occur in liquid ${ }^{3} \mathrm{He}$ and ${ }^{4} \mathrm{He}$. In solid hydrogen, since electron-phonon coupling is very large, it has been estimated(Ashcroft, 1968) that atomic hydrogen will be a room temperature superconductor.

Further motivation for studying dense hydrogen comes from technological applications, for example, inertial confinement fusion (ICF), where hydrogen gas is compressed with a laser-driven shock into the region where DT fusion could occur, at physical conditions close to that of HD209458b in Fig.1. Such aspects will not be directly addressed in this review; the reader is instead referred to Lindl et al., 2004, for example. Nonetheless, the focus of our discussion is equally pertinent.

A final theoretical motivation for studying hydrogen is to develop and test computer simulation methods. Hydrogen and helium are somewhat simpler than other elements but pose unique difficulties for simulation. Since they have no core electrons, their atomic structure is simple and the errors from the pseudopotential approxima- tion, often employed to increase computational efficiency, are significantly smaller or absent. Furthermore, relativistic effects are small, hence spin orbit effects can be ignored. However, because the protons, deuterons, and alpha particles that constitute the nuclei are so light, they too behave as quantum mechanical particles. This has a strong influence on even the most basic properties of the system, such as relative stabilities of atomic structures (Natoli et al., 1993). Harmonic corrections to account for nuclear motion do not always work in hydrogen and helium. Thus, both the electrons and the ions must be treated using quantum mechanics in order to make definitive predictions. The availability of experimental data and the intense physical interest has made the study of high pressure hydrogen and helium into a test-bed for theory and simulation. If the modern computational techniques to treat electron correlation, such as those based on quantum Monte Carlo (QMC) methods and density functional theory (DFT) are not accurate for hydrogen and helium, there are serious problems in trusting them for heavier elements.

This article concerns the thermodynamic properties of Hydrogen and Helium at pressures above $10 \mathrm{GPa}$ and for temperatures less than $100,000 \mathrm{~K}$. Our primary focus is on advanced simulation methods used for hydrogen and helium in this region of pressure and temperature and their comparison with experimental results.

We start by describing the theoretical and numerical tools that are used for describing hydrogen (Section II). We then provide a brief discussion of the experimental methods that are in use (Section III), in order to facilitate the understanding of theory vs. experiment comparisons that follow. In Section IV, we describe the current understanding of the phase diagram of hydrogen under extreme conditions and its properties and interesting predictions such as metallization, superconductivity, and the possibility of a quantum fluid ground-state. In Section V we provide a brief discussion of helium, and describe the behavior of the hydrogen-helium mixtures of primary importance to astrophysical applications. Section VI concludes and discusses some of the open questions that remain.

\section{PREDICTING PROPERTIES OF MATTER UNDER EXTREME CONDITIONS}

In this section we review some of the computational methods for hydrogen and helium at high pressures. The properties of hydrogen and helium at conditions of interest are described to high accuracy by the non-relativistic 
Hamiltonian for a collection of electrons and ions:

$$
\begin{aligned}
\hat{H} & =\hat{T}_{n}+\hat{H}_{e l}=\hat{T}+\hat{V} \\
\hat{H}_{e l} & =\hat{T}_{e}+\hat{V}_{n-n}+\hat{V}_{e-e}+\hat{V}_{e-n} \\
\hat{T} & =\hat{T}_{n}+\hat{T}_{e} \\
\hat{T}_{n} & =\sum_{I=1}^{N_{n}}-\lambda_{I} \hat{\nabla}_{I}^{2}, \quad \hat{T}_{e}=-\lambda_{e} \sum_{i=1}^{N_{e}} \hat{\nabla}_{i}^{2}, \\
\hat{V} & =\hat{V}_{n-n}+\hat{V}_{e-e}+\hat{V}_{e-n} \\
\hat{V}_{n-n} & =\sum_{I<J} \frac{z_{I} z_{J}}{\left|\vec{R}_{I}-\vec{R}_{J}\right|}, \quad \hat{V}_{e-e}=\sum_{i<j} \frac{1}{\left|\vec{r}_{i}-\vec{r}_{j}\right|}, \\
\hat{V}_{e-n} & =-\sum_{i, I} \frac{z_{I}}{\left|\vec{r}_{i}-\vec{R}_{I}\right|},
\end{aligned}
$$

where $N_{n}$ and $N_{e}$ are the number of nuclei and electrons respectively, $\lambda_{e}=1 / 2, \lambda_{I}=1 /\left(2 M_{I}\right)$ and $M_{I}$ and $z_{I}$ are the mass (in units of the electron mass $m_{e}$ ) and charge (in units of the electron charge $e$ ) of the nucleus $I^{1}$. We implicitly assume charge neutrality of the system: $\sum_{I} z_{I}=N_{e}$. We use $\vec{r}$ with lower case indexes $(i$, $j, \ldots)$ to denote the position of electrons, and $\vec{R}$ with upper case indexes $(I, J, \ldots)$ for the nuclei. When no indexes are used $\vec{r}$ and $\vec{R}$ represent the full $3 N_{e}\left(3 N_{n}\right)$ dimensional vectors. The electronic Hamiltonian in the clamped nuclei approximation where the ions produce a fixed external potential for the electrons is given by $\hat{H}_{e l}$. We always treat electrically neutral systems; hence for hydrogen the number of electrons is also $N_{n}$, while for helium it is $2 N_{n}$. The electron number density is given by $\rho$ and parameterized with $r_{s}=a / a_{0}$ where $4 \pi a^{3} / 3=\rho^{-1}$. We only need to add the temperature, particle statistics and boundary conditions to completely specify the numerical and physical problem to be solved.

Finding the eigenvalues and eigenfunctions of the Hamiltonian of Eq. (1) is a formidable task, impossible to do analytically except for the single hydrogen atom or the $\mathrm{H}_{2}^{+}$molecular ion; numerical or approximate theoretical methods are used in practice. Two of the most widely applicable methods are based on imaginary-time path integrals and on density functional theory, as discussed in the following subsections. The path integral and Monte Carlo methods for solving the fundamental equations are discussed next, followed by methods based on density functional theory. Then we briefly discuss semi-empirical methods.

\footnotetext{
${ }^{1}$ We use atomic units in the methods section, where $\hbar=m_{e}=$ $k_{B}=e=1 ; m_{e}$ is the mass of the electron, $k_{B}$ is Boltzmann's constant and the energy is measured in $E_{\mathrm{h}}=315,775 \mathrm{~K}=$ $27.2114 \mathrm{eV}$, the energy of a hydrogen atom is $0.5 E_{\mathrm{h}}$; the binding energy of a $\mathrm{H}_{2}$ molecule is $0.17 E_{\mathrm{h}}$; the unit of length is the Bohr Radius $a_{0}=0.0529 \mathrm{~nm}$, the equilibrium hydrogen bond length is $1.4 a_{0}$.
}

\section{A. The Formalism of Imaginary-time Path Integrals}

Path integrals provide a theoretical and computational framework to discuss the many-body problem. The partition function of a quantum system at an inverse temperature $\beta=1 /\left(k_{B} T\right)$ is the trace of the many-body density matrix:

$$
Z=\int d \vec{R} d \vec{r} \rho(\vec{R}, \vec{r} ; \vec{R}, \vec{r} ; \beta),
$$

where $\rho\left(\vec{R}, \vec{r} ; \overrightarrow{R^{\prime}}, \overrightarrow{r^{\prime}} ; \beta\right)$ is the density matrix in the position basis for the appropriate ensemble ${ }^{2}$. In the thermal ensemble it has the form:

$$
\rho\left(\vec{R}, \vec{r} ; \overrightarrow{R^{\prime}}, \overrightarrow{r^{\prime}} ; \beta\right)=\left\langle\vec{R}, \vec{r}\left|e^{-\beta \hat{H}}\right| \overrightarrow{R^{\prime}}, \overrightarrow{r^{\prime}}\right\rangle .
$$

The equilibrium average of an operator $\hat{O}$ is computed as:

$$
\begin{gathered}
\langle\hat{O}\rangle_{\rho}=Z^{-1}\langle\hat{O} \hat{\rho}\rangle= \\
Z^{-1} \int d \vec{R} d \vec{r} d \overrightarrow{R^{\prime}} d \overrightarrow{r^{\prime}} \rho\left(\vec{R}, \vec{r} ; \overrightarrow{R^{\prime}}, \overrightarrow{r^{\prime}} ; \beta\right)\left\langle\overrightarrow{R^{\prime}}, \overrightarrow{r^{\prime}}|\hat{O}| \vec{R}, \vec{r}\right\rangle .
\end{gathered}
$$

The product property of the exponential of commuting operators:

$$
e^{-\left(\beta_{1}+\beta_{2}\right) \hat{H}}=e^{-\beta_{1} \hat{H}} e^{-\beta_{2} \hat{H}},
$$

repeatedly applied, gives the path integral expression for the partition function:

$$
Z=\int \prod_{t=0}^{P-1} d \vec{R}^{t} d \vec{r}^{t}\left\langle\vec{R}^{t}, \vec{r}^{t}\left|e^{-\tau \hat{H}}\right| \vec{R}^{t+1}, \vec{r}^{t+1}\right\rangle
$$

where $\tau=\beta / P$ and periodic boundary conditions in the index $t$ applies: $\vec{R}_{0}=\vec{R}_{P}, \vec{r}_{0}=\vec{r}_{P}$. To account for Bose or Fermi statistics, a permutation of identical particles can also be applied as we note below.

We define the path as $\overrightarrow{\mathcal{R}}=\left\{\vec{R}^{0}, \vec{r}^{0}, \ldots \vec{R}^{P}, \vec{r}^{P}\right\}^{3}$; it consists of $3 P\left(N_{e}+N_{n}\right)$ variables. This expression, exact for any value of $P$ (the number of time slices or beads), allows us to compute properties of a quantum system at inverse temperature $\beta$ using a density matrix evaluated at a smaller inverse temperature, $\tau=\beta / P$. At small enough $\tau$, accurate and computationally simple approximations exist for the thermal density matrix which become exact as $\tau \rightarrow 0$. The best known example is the Trotter formula (Trotter, 1959):

$$
e^{-\beta \hat{H}}=\lim _{P \rightarrow \infty} \prod_{P=0}^{P-1} e^{-\tau \hat{T}} e^{-\tau \hat{V}}
$$

\footnotetext{
2 For simplicity, we limit our discussion to the canonical ensemble. It is straight-forward to extend the discussion to other ensembles.

3 Superscripts will label imaginary time indexes to avoid confusion.
} 
one can ignore the commutator of the kinetic and potential operators in writing an integral expression for the density matrix at large $P$. Using the Trotter formula, the density matrix becomes: (Ceperley, 1992, 1996; Feynman, 1972).

$$
\rho\left(\vec{R}^{0}, \vec{r}^{0} ; \vec{R}^{0}, \vec{r}^{0} ; \beta\right)=\frac{1}{N !} \sum_{\mathcal{P}}( \pm 1)^{\mathcal{P}} \int_{\vec{R}^{0} \rightarrow \mathcal{P} \vec{R}^{0}} d \overrightarrow{\mathcal{R}} e^{-S(\overrightarrow{\mathcal{R}})}
$$

where we have include particle statistics (Bose or Fermi) by summing over permutations when the path is closed. $\mathcal{P}$ labels the permutation of the particles and $( \pm 1)^{\mathcal{P}}$ is its signature for bosons $(+)$ or fermions $(-)$. The "action" (the logarithm of its probability density) of the path $S(\overrightarrow{\mathcal{R}})$ is given (neglecting a constant term) by:

$$
\begin{aligned}
& S(\overrightarrow{\mathcal{R}})= \\
& \int_{0}^{\beta} d t\left[\frac{M_{n}}{2}\left|\frac{d \vec{R}(t)}{d t}\right|^{2}+\frac{1}{2}\left|\frac{d \vec{r}(t)}{d t}\right|^{2}+V(\vec{R}(t), \vec{r}(t))\right](9) \\
\simeq & \sum_{t=0}^{P-1}\left[\frac{M_{n}\left|\vec{R}^{t}-\vec{R}^{t+1}\right|^{2}}{2 \tau}+\frac{\left|\vec{r}^{t}-\vec{r}^{t+1}\right|^{2}}{2 \tau}+\tau V\left(\vec{R}^{t}, \vec{r}^{t}(1)\right],\right.
\end{aligned}
$$

Equation (9) represents the exact expression in the continuous path limit $(P \rightarrow \infty, \tau \rightarrow 0, P \tau=\beta)$. To simplify the notation, we considered a system with a single nuclear component of mass $M_{n}$. Nuclei, being heavier, are represented by paths of much smaller size. For bosons (positive sign), or boltzmannons (no sum over permutations), the integrand in Eq. (8) is positive, and the partition function becomes identical to the Boltzmann distribution of a classical system of $\left(N_{e} \times N_{n}\right) \times P$ particles interacting with an effective classical "potential" $k_{B} T S(\mathcal{R})$.

The electronic integral can be formally performed producing the influence functional, $Z_{e l}\left[\overrightarrow{\mathcal{R}}_{n}\right]$ :

$$
\begin{aligned}
Z & =\oint \mathcal{D} \vec{R} \oint \mathcal{D} \vec{r} \exp \left[-\int_{0}^{\beta} d t\left(T_{n}\left(\vec{R}^{t}\right)+S_{e l}\left(\vec{R}^{t}, \vec{r}^{t}\right)\right)\right] \\
& =\oint \mathcal{D} \vec{R} \exp \left[-\int_{0}^{\beta} d t T_{n}\left(\vec{R}^{t}\right)\right] Z_{e l}\left(\overrightarrow{\mathcal{R}}_{n}(t),\left[\overrightarrow{\mathcal{R}}_{n}\right]\right)
\end{aligned}
$$$$
Z_{e l}\left(\overrightarrow{\mathcal{R}}_{n}(t),\left[\overrightarrow{\mathcal{R}}_{n}\right]\right)=\oint \mathcal{D} \vec{r} \exp \left[-\int_{0}^{\beta} d t S_{e l}\left(\vec{R}^{t}, \vec{r}^{t}\right)\right]
$$

where $\oint \mathcal{D} \vec{R}(\oint \mathcal{D} \vec{r})$ is a short hand notation for the functional integral over all paths of length $\beta, \overrightarrow{\mathcal{R}}_{n}$ represents the ionic coordinates of the path $\overrightarrow{\mathcal{R}}$, and the meaning of $T_{n}$ (related to the nuclear kinetic energy operator in the Hamiltonian) and $S_{e l}$ should be clear from Eqs. (1) and (9). Eq. (11) is just a formal manipulation of the partition function but it provides a useful framework to understand the Born-Oppenheimer approximation introduced below. Note that $Z_{e l}\left(\overrightarrow{\mathcal{R}}_{n}(t),\left[\overrightarrow{\mathcal{R}}_{n}\right]\right)$ is a functional of the nuclear path, $\overrightarrow{\mathcal{R}}_{n}$, a fact which becomes important below, and also a function of imaginary time through its explicit dependence on the nuclear path coordinates.

Eq. (10) is the simplest approximation to the high temperature density matrix commonly employed in numerical calculations. In principle, any approximation correct to first order in imaginary time will give the same result as $\tau \rightarrow 0$. But for computational efficiency and stability for path integral simulations, one can use improved actions some of which include semiclassical expressions, higher order expansions (De Raedt and De Raedt, 1983; Takahashi and Imada, 1984), cumulant expansions (Ceperley, 1995), and pair product actions (Barker, 1979; Ceperley, 1995). In most cases, improved actions lead to dramatic reductions in the numerical effort required to solve quantum problems. Of particular importance to care of is the divergence in the potential as two charged particles approach each other.

Consider now the effect of incorporating the quantum statistics of the nuclei. The temperature $T_{Q}$, when quantum statistics becomes relevant can be estimated by setting the thermal de Broglie wavelength (proportional to $\left.\left(k_{B} T_{Q} M_{n}\right)^{-1 / 2}\right)$ to the average spacing between the ions given by $r_{s}$. One can show that for ideal fermions this corresponds to the Fermi energy up to a factor of order unity. For $3 \mathrm{D}$ protons we have $T_{Q}=\left(m_{e} / M_{p}\right) r_{s}^{-2} 5.84 R y=504 K / r_{s}^{2}$. At the metalinsulator transition $\left(r_{s} \simeq 1.4\right), T_{Q} \simeq 250 \mathrm{~K}$. In fact, the effects of quantum statistics for the protons are smaller than this estimate because the correlation between protons makes their exchange less probable than for noninteracting protons. Above the temperature, $T_{Q}$ we can safely ignore the quantum statistics of the nuclei (FermiDirac for hydrogen, Bose-Einstein for deuterium) and treat them as distinguishable particles.

To turn the path integral expression into a numerical procedure, generalizations of classical simulation methods developed to perform high dimensional integrals and based on the Monte Carlo method are used. In the following subsections, we will describe the three main Quantum Monte Carlo methods that have been used to treat dense hydrogen and helium: path integral Monte Carlo approaches for simulations at non-zero temperature; zero temperature wavefunction-based approaches including variational, reptation and diffusion Monte Carlo; and a method that explicitly separates electron and ionic degrees of freedom, the Coupled-Electron Ion Monte Carlo. However, before discussing the numerical methods we discuss the Born-Oppenheimer (BO) approximation which is used in one of the Quantum Monte Carlo methods to be discussed next, and in the First Principle Molecular Dynamics methods based on Density Functional Theory which we will review in some detail in section II.E. 


\section{B. The Born-Oppenheimer Approximation}

Electronic motion is much faster than that of the nuclei. The implications of the resulting separation in time scale were recognized in the early years of quantum mechanics, and led to the development of the BornOppenheimer (BO) approximation (Born and Oppenheimer, 1927). It is based on the fact that the proton is considerably heavier than the electron: $M_{p} / m_{e} \approx 1836$; all other nuclei are much heavier. Since the velocity of electrons is much higher than that of ions, we can assume that electrons relax instantaneously and adiabatically to their equilibrium state as the ions move, neglecting any coupling between different electronic states, and also retardation effects in the electron-ion interaction. This leads to a partial decoupling of the electronic and ionic problems: the ions move in an effective potential defined by the solutions of the electronic hamiltonian for fixed ionic positions.

In the $\mathrm{BO}$ approximation, the partition function can be expressed in path integral notation (Feynman and Hibbs, 1965)

$Z_{B O}=\sum_{q} \oint \mathcal{D} \vec{R} \exp \left[-\int_{0}^{\beta} d t\left(T_{n}(\vec{R}(t))+E_{q}(\vec{R}(t))\right)\right]$

where the sum is over a complete set of electronic states of $\hat{H}_{e l}$ for a fixed set of ionic positions with eigenvalues $E_{q}(\vec{R})$ :

$$
\hat{H}_{e l}(\vec{r}, \vec{R}) \Phi_{q}(\vec{r}, \vec{R})=E_{q}(\vec{R}) \Phi_{q}(\vec{r}, \vec{R}) .
$$

If the temperature is low enough to neglect excited electronic states in Eq. (12), the standard form of the BO approximation is obtained, where the ions move in the potential energy surface defined by the electronic ground state energy $E_{0}(\vec{R})$. For metals and systems at high temperatures, we must take into account the sum over electronic states. In that case, Eq. (12) would require a diagonalization of $\hat{H}_{e l}$ for every position of the ions, including all relevant electronic eigenstates. Following the work of Cao et al.(Cao and Berne, 1993), a simpler expression can be obtained by factorizing the exponential terms and performing the sum over electronic eigenstates, with the added complication that, in general, $T_{n}$ and $E_{q}$ do not commute. This leads to the following approximation to the partition function:

$$
Z_{F E B O}=\oint \mathcal{D} \vec{R} \exp \left[-\int_{0}^{\beta} d t\left(T(\vec{R})+F_{e l}(\vec{R}(t))\right)\right]
$$

where we neglected terms of order $\mathrm{O}\left[m_{e} / M_{I}\right]$, see Cao and Berne, 1993 for additional details. Equation (14) is known as the Free-Energy Born-Oppenheimer (FEBO) approximation since the electronic Free Energy along the nuclear path, $F_{e l}(\vec{R})$, at temperature $\beta$ appears. Note that in this approximation, the influence functional has become a simple function of the nuclear path, loosing the non-local (in imaginary time) dependence

Eq. (14) is the main result on this section; we managed to replace the potential energy operator with a local function of the ionic coordinates: the BO free energy surface. By employing the BO approximation, we have decoupled the problem into two parts, making a great simplification at the expense of a good approximation. The electronic problem has been completely encapsulated in the potential energy surface (PES), and can be solved with any suitable approximate method such as Density Functional Theory (DFT) or Quantum Monte Carlo (QMC). Once the PES has been defined, the ionic problem can be solved using path integrals for quantum ions or classical Monte Carlo or Molecular Dynamics for classical ions.

Note that FEBO formulation provides a unifying framework to interpret several apparently different approximations routinely used in first-principle simulation methods. Consider thermodynamic conditions at which protons can be treated as classical particles. In the atomic phase, these conditions are realized for $T$ larger than $T_{Q}$ defined above For example, at $r_{s} \simeq 1.4$, nuclear quantum effects are negligible for $T \gtrsim 1000-2000 K$. However, in the molecular phase, the bonding potential is much stronger than the intermolecular interaction and produces much larger quantum effects which results in a vibrational temperature of $\sim 6000 K$ in the isolated hydrogen molecule, and even higher values at finite density . However, the rotational temperature of a molecule is only $\sim 100 K$. Therefore, in the molecular phase, quantum effects are important even at relatively high temperature, a fact often neglected in the applications. When nuclear quantum effects can be neglected, nuclear "imaginarytime paths" in Eq. (12) shrink to a single point and the electronic term appearing in the partition function is simply the exponential of the electronic free energy function of the nuclear configuration. If, furthermore, thermal occupation of the electronic states can be neglected, the electronic free energy reduces to the ground state electronic energy, and we recover the standard BO approximation. Conversely, if the temperature is low enough that neglecting thermal occupation of the electronic states is an accurate approximation, and nuclear quantum effects need to be taken into account, the sum over electronic states in Eq. (12) drops out and the action for the ionic paths depends only on the electronic ground state PES. This is the BO approximation for quantum ions. Note that in the implementation of the numerical methods discussed below, in particular, in the DFTbased methods applied to metals, thermal occupation of the electronic states is necessary to ensure the stability of the self-consistent procedure. In view of our discussion, this fact seems to be more fundamental than simply a technical tool. 


\section{Path Integral Monte Carlo}

Path integral methods for bosons and distinguishable particles are quite well developed(Ceperley, 1995) since there is no fermion minus sign. There are two main computational problems: choice of the action, and sampling of the paths. As mentioned in section II.A, there are several options for the path integral action with varying degrees of efficiency and convergence properties. For hydrogen and helium, the optimal strategy has been the use of pair density matrices, where the two body problem is solved exactly at very high temperature and the matrix squaring method is used to produce a density matrix at the desired temperature. Using the "pair action" instead of the bare potential reduces the number of time slices, and eliminates the instability that would otherwise occur in the primitive approximation to the path integral for the hydrogen atom caused by the potential going to $-\infty$ as an electron approaches a proton. The sampling of the paths is performed using a generalized Metropolis (Markov chain Monte Carlo) procedure. This is the only effective algorithm when the paths become very long, e.g. for electrons or at low temperatures. Special methods are used to change the permutation variables. See ref. (Ceperley, 1995). As mentioned in the last subsection, as long as the temperature is high enough $(T>100 K$ for hydrogen in the metallization region, $P \simeq 100 G P a$ ) nuclear exchange is irrelevant and only the identity permutation has a significant contribution to the partition function. In this case, ionic paths are isomorphic to ring polymers. When the path integral representation is used for the ions and the electrons are treated with other methods (ground state QMC or DFT), nuclear paths can also be sampled by molecular dynamics; see section II.E.5 for more details.

For electronic systems, it is possible to perform PIMC simulations without invoking to the BO approximation. Both electrons and ions are represented in the path integral expression, Eq. (8). Notice that this is probably the only way to simulate directly electron-ion systems at finite temperature without approximations. In practice, the integration is complicated due to the cancelation of positive and negative contributions from the negative sign of the permutation, (the fermion sign problem). The efficiency (inverse of computer time needed to reach a given precision) of treating this exactly scales like $\exp \left(-2 \beta\left(F_{F}-F_{B}\right)\right)$ where $\left(F_{F}-F_{B}\right)$ is the free energy difference between the fermi and bose system; the exponent is extensive in the size of the system (Ceperley, 1996). Because of this, in the direct approach, one cannot treat systems where the number of electrons is large or the temperature is comparable with the Fermi energy.

However, it has been shown (Ceperley, 1992, 1996) that one can evaluate the path integral by restricting the path to only positive contributions. One introduces a reference point $\vec{R}^{*}$ on the path that specifies the nodes of the fermion density matrix, $\rho\left(\vec{R}, \vec{R}^{*}, t\right)=0$ with respect to the reference point $\vec{R} *$. A node-avoiding path for $0<t \leq \beta$ never crosses a node: $\rho\left(\vec{R}(t), \vec{R}^{*}, t\right) \neq 0$. By restricting the integral to these paths,

$$
\begin{aligned}
\rho_{F}\left(\vec{R}_{\beta}, \vec{R}^{*} ; \beta\right) & = \\
\int d \vec{R}_{0} & \rho_{F} \quad\left(\vec{R}_{0}, \vec{R}^{*} ; 0\right) \underset{\vec{R}_{0} \rightarrow \vec{R}_{\beta} \in \Upsilon\left(\vec{R}^{*}\right)}{\oint d \vec{R}_{t}} e^{-S\left[\vec{R}_{t}\right]},
\end{aligned}
$$

$\left(\Upsilon\left(\vec{R}^{*}\right)\right.$ denotes the restriction) the contributions to the partition function are strictly positive. This, therefore represents, in principle, a solution to the sign problem, but only if the exact fermionic density matrix is used for the restriction. In practice, one must approximate the density matrix. The simplest approximation is to use a determinant of single particle density matrices:

$$
\rho\left(\vec{R}, \vec{R}^{\prime} ; \beta\right)=\left|\begin{array}{ccc}
\rho_{1}\left(\vec{r}_{1}, \vec{r}_{1}^{\prime} ; \beta\right) & \ldots & \rho_{1}\left(\vec{r}_{N}, \vec{r}_{1}^{\prime} ; \beta\right) \\
\ldots & \ldots & \ldots \\
\rho_{1}\left(\vec{r}_{1}, \vec{r}_{N}^{\prime} ; \beta\right) & \ldots & \rho_{1}\left(\vec{r}_{N}, \vec{r}_{N}^{\prime} ; \beta\right)
\end{array}\right|
$$

It can be shown that for temperatures larger than the Fermi energy, the interacting nodal surface approaches the free particle nodal surface, i.e. the nodes of the determinant in Eq. (16) when free particle (FP) single particle density matrices are used

$$
\rho_{1}\left(\vec{r}, \vec{r}^{\prime}, \beta\right)=(2 \pi \beta)^{-3 / 2} \exp \left\{-\left(\vec{r}-\vec{r}^{\prime}\right)^{2} / 2 \beta\right\}
$$

At low density, exchange effects are unimportant, for example, in the molecular phase, when electrons are localized in a molecule in a nodeless spin singlet state. However, in general, at temperatures much less than the Fermi energy, interactions could have a significant effect on the nodal surfaces and hence on which paths are allowed. Several methods have been developed to go beyond the FP restriction. In Militzer and Ceperley, 2000, the nodal surface of a density matrix derived from a variational principle (VDM) that includes interparticle interactions and electronic bound states was used. Also recently Khairallah et al., 2011 implemented a modified nodal restriction, the so-called antinodal-slice constraint. This method has the advantage of eliminating the reference point and thereby allowing the paths to be much more easily sampled than those that have a reference point, however, it introduces a systematic approximation which does not become exact even if exact nodes would be employed. Note that in these methods, the trial density matrix is only used to determine the nodal constraint; the complete potential is taken into account in the path integral action (Ceperley, 1995). Although the PIMC fermion methods have not been extensively used, they show great promise for the study of more complex materials at high temperature (see for instance a recent application to water and carbon by Driver and Militzer, 2012).

An alternative simulation method based on path integrals for electron-ion systems, called the Direct Path Integral Monte Carlo (DPIMC) method, has been developed over the last decade by Filinov et al., 2005. At variance with the RPIMC, Fermi statistics for the electrons 
is accounted by inserting a Slater determinant of single particle propagators in the last link of the electronic paths. The sampling of the fermionic loops is based on the absolute value of the determinant and the sign of the determinant is used for the estimator. This method is adequate in the semiclassical regime where the effect of Fermi statistics is marginal. However, it has a sign problem which will become more and more serious when approaching the quantum regime. For a given Hamiltonian, the probable phase space for fermions is very different from that of bosons; sampling by a bosonic density matrix will produce paths irrelevant for the fermions. This might be at the origin of formation of large density inhomogeneities observed by this method when lowering the temperature(Filinov et al., 2003). Moreover, the systematic effect of finite imaginary time step seems not to have been considered adequately in the original publications (Filinov et al., 2005).

\section{QMC-based First-Principles simulations}

Once the BO approximation is employed, the electronic calculation can be solved with any appropriate electronic structure method. The most common approach is to use Density Functional Theory; this leads to methods typically known as Ab-Initio Molecular Dynamics or First-Principles Molecular Dynamics. Section II.E gives a detailed description of DFT-based approaches. A more advanced approach consists in using QMC methods to solve the electronic problem. QMC, although more accurate than DFT in general, is also more expensive. As a consequence, only a small number of first-principles studies using QMC have been reported in the literature, all of them on high pressure hydrogen and helium (Attaccalite and Sorella, 2008; Ceperley et al., 2002; Delaney et al., 2006; Morales et al., 2010a; Pierleoni and Ceperley, 2005a, 2006; Pierleoni et al., 2004, 2008). This should be compared with the thousands of DFT-based FP simulations published so far (Marx and Hutter, 2000). In the framework of QMC-based simulations at non-zero temperature, only two approaches have been tried. In the Coupled Electron-Ion Monte Carlo method (CEIMC), discussed below, QMC methods are used to calculate energy differences during a Metropolis MC simulation of the nuclei. This method has been recently used to study the equation of state and the liquid-liquid transition in hydrogen (Morales et al., 2010b), it has also been used to study hydrogen-helium mixtures (Morales, 2009) and to perform free energy calculations (Liberatore et al., 2011a; Morales, 2009; Morales et al., 2010a). An alternative MD approach based on a "noisy" Langevin dynamics scheme, with ionic forces from QMC, has also been developed (Attaccalite and Sorella, 2008) and applied to high pressure hydrogen.

Before discussing QMC-based first-principle methods to simulate finite temperature systems, we provide a detailed description of ground state QMC methods below.
These methods can be either used to obtain an accurate solutions of the ground state electronic problem in the finite temperature QMC-based FP methods in the framework of the BO approximation, or can be used, without resorting to the $\mathrm{BO}$ approximation, to obtain accurate solutions for electron-ion systems in their ground state.

\section{Ground-state Quantum Monte Carlo methods}

Ground state quantum Monte Carlo (QMC) methods were the first calculations that applied quantum simulation techniques to a many-body crystal including both the electronic and ionic degrees of freedom (Ceperley and Alder, 1981, 1987a). The ultimate goal of QMC is to provide an exact stochastic solution to the Schrödinger equation, similar to the way Monte Carlo methods can be used to solve classical many body problems. In practice, approximations must be used to treat fermions. The main advantage of QMC over alternative methods for electronic structure calculations is the balance that it provides between accuracy and computational cost. Currently, QMC can provide results that are more accurate than DFT for about an order of magnitude additional computational cost and QMC scales approximately as $N^{2}$ to $N^{3}$ with the number of electrons. Traditional chemistry methods, while providing a higher degree of accuracy in general, have a very unfavorable scaling and as a consequence can only be applied to few electron systems. QMC, on the other hand, can be applied to systems with up to several thousand electrons with currently available computational resources.

Variational Monte Carlo. The simplest quantum simulation, variational Monte Carlo (VMC), was first introduced by McMillan (McMillan, 1965) for liquid helium (modeled as a single composite particle) and generalized to fermions by Ceperley et al., 1977. VMC allows us to optimize the trial function needed in the projector methods, described below. Let $\psi_{T}(\vec{R})$ be an assumed trial function with adjustable parameters $\{a\}$. Using the Metropolis Monte Carlo technique (Metropolis et al., 1953), one samples the un-normalized distribution, $\left|\psi_{T}(\vec{R})\right|^{2}$ and calculates an upper bound to the ground state energy.

$$
\begin{aligned}
E \leq E_{V}(a) & =\frac{\int d \vec{R} \Psi_{T}^{*}(\vec{R}) \hat{H} \Psi_{T}(\vec{R})}{\int d \vec{R} \Psi_{T}^{*}(\vec{R}) \Psi_{T}(\vec{R})} \\
& =\frac{\int d \vec{R}\left|\Psi_{T}(\vec{R})\right|^{2} E_{L}(\vec{R})}{\int d \vec{R}\left|\Psi_{T}(\vec{R})\right|^{2}}
\end{aligned}
$$

The fluctuations in the energy estimator, the local energy $E_{L}(\vec{R})=\left[\hat{H} \Psi_{T}\right] / \Psi_{T}$, are entirely due to inaccuracies of the trial function: as the trial wave function approaches the exact eigenfunction, the fluctuations, which control how quickly the energy converges, decrease to zero. At the same time, the estimate converges to the exact energy. 
The Trial Function. The strategy in Variational Monte Carlo (VMC) is to pick a form for the trial wave function that incorporates as many properties of the exact many-body wave function as possible, while maintaining enough flexibility to allow for its efficient optimization. The pair product trial wave function is the simplest extension of the Slater determinant of single particle orbitals used in mean field treatment of electronic systems:

$$
\Psi_{S J}(\vec{R})=\exp \left(-\sum_{i<j} u\left(r_{i j}\right)\right) \operatorname{det}\left[\phi_{k}\left(\vec{r}_{i}, \sigma_{i} \mid \vec{R}\right)\right]
$$

where $\phi_{k}(\vec{r}, \sigma \mid \vec{R})$ is the $k^{t h}$ spin orbital for a given configuration of the protons. The term $u_{i j}\left(r_{i j}\right)$, the (Jastrow) correlation factor, introduces two-body correlations into the many body wave function. It is symmetric under particle exchange; antisymmetry is given by the determinant only. In the above trial function, we have assumed that the Jastrow factor $u(r)$ only depends on the distance between two electrons; more generally it can also depend on the positions of the nuclei and their spin states. Often either or both of $\phi_{k}$ and $u_{i j}$ are derived from an approximate theory such as the Random Phase Approximation (for $u$ ) or DFT (for $\phi$ ).

The spin-orbitals are quite important because they provide the nodal structure of the trial function crucial in the fixed node approximation described below. The choice of orbitals for hydrogen has evolved over the years. The first QMC calculation of metallic hydrogen (Ceperley and Alder, 1981, 1987a) used plane waves (PW) $\theta_{\vec{k}}(\vec{r}, \sigma)=\exp [\imath \vec{k} \cdot \vec{r}]$. Although they are qualitatively correct in the metallic phase, since the electron-proton "cusp" can be taken into account in the correlation factor $u(r)$, quantitatively accurate results require better orbitals that include information about the positions of the ions.

The next step in development came with the work of Natoli et al., 1993, 1995; Wang et al., 1990, where orbitals obtained from a band structure calculations were employed. Natoli and Ceperley, 1995 established that energies from plane-waves determinants in metallic hydrogen are higher than the values using DFT-LDA orbitals by $0.05 \mathrm{eV} /$ atom at the density at which the transition between molecular and metallic hydrogen is expected $\left(r_{s}=1.31\right)$.

Turning now to the correlation factors, $u_{i j}\left(r_{i j}\right)$, optimal factors will obey the "cusp" condition at short distances

$$
\left.\frac{d u_{i j}}{d r}\right|_{0}=-\alpha_{i j}
$$

where $\alpha_{i j}=1 / 2$ if $i$ and $j$ have antiparallel spins, otherwise $\alpha_{i j}=1 / 4$. Within the Random Phase Approximation (RPA), the correlation factors for the charged particles have the fourier transforms:

$$
u_{k}=-\frac{1}{2}+\sqrt{1+\frac{12 r_{s}}{k^{4}}}
$$

This forms has the exact behavior at both small and large distances for a metallic system. It is important to reproduce the correct $1 / r$ behavior at large distances since that controls the dielectric properties. In practice, these RPA functions are typically augmented with flexible forms that preserve the asymptotic behaviors, e.g. gaussians, but contain free parameters which can be tuned to improve the trial function.

The first corrections to the pair product trial function are a three-body correlation term which modifies the correlation part of the trial function (Jastrow) and a "backflow" transformation which changes the orbitals and therefore the nodal structure (or the phase) of the trial function (Holzmann et al., 2003; Kwon et al., 1993; Pierleoni et al., 2008). This transformation introduces correlation effects into the Slater determinant; the energy and the nodal surfaces of the trial function are improved. The modified trial function has the form

$$
\Psi_{T}(\vec{R}, \Sigma \mid S)=\operatorname{det}\left[\theta_{k}\left(\vec{x}_{i}, \sigma_{i}\right)\right] e^{-U_{2}-U_{3}}
$$

where $U_{2}=\sum_{i<j} \tilde{u}\left(r_{i j}\right)$ is the two body correlation factor discussed before (the indicates that it can differ from the original one, (see Holzmann et al., 2003 for details), $U_{3}$ is a three-body term of the form

$$
U_{3}=-\sum_{i=1}^{N_{e}}\left[\sum_{j=1}^{N} \xi_{i j}\left(r_{i j}\right) \vec{r}_{i j}\right]^{2}
$$

and finally the "quasiparticle" coordinates appearing in the plane wave orbitals are given by

$$
\vec{x}_{i}=\vec{r}_{i}+\sum_{j=1}^{N} \eta_{i j}\left(r_{i j}\right) \vec{r}_{i j} ; \quad\left(i=1, \cdots, N_{e}\right)
$$

The RPA approximation has been used (Holzmann et al., 2003) to provide a general form for the functions $\xi_{i j}(r), \eta_{i j}(r)$ in Eqs. (23-24), satisfying the correct limiting behavior at small and large distances. These functions can also be augmented with flexible functional forms and optimized with VMC.

As discussed above, the trial wave function generally has variational parameters that need to be optimized at the VMC level. The optimization not only improves the quality of the final results in projector Monte Carlo methods, but also improves the efficiency of QMC calculations, which is directly related to the error of the trial wave function. Optimization methods in QMC have a long history (Drummond and Needs, 2005; Toulouse and Umrigar, 2007; Umrigar et al., 1988), with methods ranging from variance minimization, energy optimization, to a combination of both. They have received considerable attention over the last decade leading to the development of fairly robust and efficient approaches, like the linear method of Umrigar et al., 2007, which are capable not only of optimizing all the parameters in the wave function, but also of handling extremely flexible wave 
functions with thousands of variational parameters, (Attaccalite and Sorella, 2008; Clark et al., 2011). Several forms of trial wave functions beyond the Slater-Jastrowbackflow from have been explored in recent years with some success, including pfaffians (Bajdich et al., 2006) and correlated geminals (Casula et al., 2004).

Projector Monte Carlo. We now describe how to go beyond VMC by applying a function of the Hamiltonian to project out the ground state. Because Diffusion Monte Carlo (DMC) has been extensively reviewed elsewhere, (e.g. Foulkes et al., 2001), here we discuss a more recent technique, Ground State Path Integral Monte Carlo (GSPI), also known as Reptation MC. Let us define the following quantity, formally similar to a partition function:

$$
Z(t)=\left\langle\Psi_{T}\left|e^{-t \hat{H}}\right| \Psi_{T}\right\rangle=\left\langle\Psi_{T}(t / 2) \mid \Psi_{T}(t / 2)\right\rangle .
$$

Here, the projection time $t$, plays the role of the inverse temperature. The variational energy of $\Psi_{T}(t / 2)$ at time $t$ is the derivative of the logarithm of $Z(t)$ :

$$
E(t)=-\frac{\partial}{\partial t} \ln Z(t)=\frac{\langle\Psi(t / 2)|\hat{H}| \Psi(t / 2)\rangle}{\langle\Psi(t / 2) \mid \Psi(t / 2)\rangle}
$$

and the "variance" of $\Psi_{T}(t / 2)$, given as

$$
\sigma_{E}^{2}(t)=-\frac{\partial}{\partial t} E(t)=\left\langle(\hat{H}-E(t))^{2}\right\rangle \geq 0
$$

is non-negative implying that the energy decreases monotonically with time. The exact ground state is reached at large time

$$
\begin{aligned}
& \lim _{t \rightarrow \infty} E(t)=E_{0} \\
& \lim _{t \rightarrow \infty} \sigma^{2}(t)=0
\end{aligned}
$$

as long as the trial wave function has a non-zero overlap with the ground state wave function. Writing $Z(t)$ in coordinate space

$$
Z(t)=\int d \vec{r}_{1} d \vec{r}_{2} \Psi_{T}^{*}\left(\vec{r}_{1}\right) \rho\left(\vec{r}_{1}, \vec{r}_{2}, t\right) \Psi_{T}\left(\vec{r}_{2}\right)
$$

where $\rho\left(\vec{r}_{1}, \vec{r}_{2}, t\right)=\left\langle\vec{r}_{1}|\exp (-t \hat{H})| \vec{r}_{2}\right\rangle$ is the many-body thermal density matrix in coordinate space.

Thus, in order to compute any average over the ground state we need to know the thermal density matrix at large enough projection time. As we did earlier with Path Integrals, this is accomplished by breaking the projection into many small steps $\exp (-t H)=\exp (-\tau H)^{n}$, writing an explicit form for $\exp (-\tau H)$ and performing the needed integrals with Monte Carlo. The difference with PIMC is in the boundary conditions of the paths; instead of the paths closing on themselves, the paths are open and projected onto a trial function at both ends. If the trial wave function $\Psi_{T}$ is accurate, GSPI computationally is a much more efficient way of computing ground state properties than is PIMC.
As usual, the main difficulty is the fermion sign problem. For electrons, the trial wavefunction must be antisymmetric if two electrons with the same spin are exchanged. In general, the trial function is complexedvalued. Hence the integrand of Eq. (31) is not necessarily positive. One could carry along the phases of the trial functions at the two ends as part of the calculated average, however, this becomes statistically very noisy and thus inefficient at large projection time and for many electrons. This is the "sign problem" for projector Monte Carlo. The solution to this problem is the fixed-node (for real $\Psi_{T}$ ) or fixed-phase (for complex $\Psi_{T}$ ) approximation: one requires that the unknown solution have the same phase as the trial function. The resulting solutions, although approximate and dependent on the quality of the nodes of the trial wave function, are typically very good. The efficiency of the method is also reasonable for many-particle systems. The approximate results give an upper bound to the exact energy, the best upper bound with the assumed phase, and hence, the exact result if the assumed phase (or nodes) is exact.

The main advantage of GSPI is the fact that observables other than the energy are readily available from the simulation, as opposed to other projector methods such as DMC where further work is needed to obtain most observables. A crucial aspect of the GSPI method is the way the paths are sampled; sampling can become extremely inefficient. An important step forward was made with the development of the bounce algorithm (Pierleoni and Ceperley, 2005a,b) which, with respect to the standard reptation algorithm previously used, allows for the efficient exploration of phase space, and significantly reduces the probability of obtaining persistent configurations.

\section{Coupled Electron-Ion Monte Carlo (CEIMC)}

The CEIMC method is based on the BO approximation. The nuclei are treated at finite temperature, $T$, using a Metropolis MC algorithm and the electrons are treated at zero temperature using a ground state QMC method. The method can be applied to both classical and quantum ions in the path integral representation, by sampling the ionic paths from the appropriate distributions (Pierleoni and Ceperley, 2005b; Pierleoni et al., 2004). The acceptance probability in the Metropolis method is given by:

$$
A\left(\vec{R} \rightarrow \vec{R}^{\prime}\right)=\min \left[1, \exp \left(-\beta \Delta E_{B O}\right)\right],
$$

where $\vec{R}$ represent here the set of nuclear coordinates. For simplicity, we assume a uniform a-priori transition matrix and $\Delta E_{B O}=E_{B O}\left(\vec{R}^{\prime}\right)-E_{B O}(\vec{R})$ is the difference in BO energy between nuclear states $\vec{R}$ and $\vec{R}^{\prime}$ In the case of quantum ions, $\Delta E_{B O}$ is the change in the action of the associated classical system; see equation (10). In CEIMC, the estimate of $\Delta E_{B O}$ for a given trial function is computed by QMC and is therefore affected by statistical noise, which, if ignored, will bias the Metropolis 
random walk. Since the noise in the energy difference decreases with the number of Monte Carlo steps as $1 / \sqrt{N}$, reducing the noise level by direct simulation to the point where the bias is negligible is time consuming. To solve this problem the Penalty Method is used (Ceperley and Dewing, 1999).

The basic idea of the penalty method is to relax the requirement of detailed balance for every step in the simulation and instead require detailed balance to hold only when we average over the noise distribution. Consider two ionic states $\left(\vec{R}, \vec{R}^{\prime}\right)$ and call $\delta\left(\vec{R}, \vec{R}^{\prime}\right)$ the "instantaneous" energy difference multiplied by $\beta=\left(k_{B} T\right)^{-1}$. Further assume that the average and the variance of $\delta\left(\vec{R}, \overrightarrow{R^{\prime}}\right)$ over the noise distribution $P\left(\delta \mid \vec{R} \rightarrow \vec{R}^{\prime}\right)$ exist: Since the noise is normally distributed because of the Central Limit Theorem (assuming the variance of $\delta\left(\vec{R}, \vec{R}^{\prime}\right)$ is finite) (Feller, 1968), it can be shown (Ceperley and Dewing, 1999) that accepting the moves according to:

$$
a\left(\delta, \chi^{2}, n\right)=\min \left[1, \frac{T\left(\vec{R}^{\prime} \rightarrow \vec{R}\right)}{T\left(\vec{R} \rightarrow \vec{R}^{\prime}\right)} \exp \left(-\delta-u_{B}\right)\right],
$$

where $u_{B}$ is a correction because the variance is also estimate from the data:

$$
u_{B}=\frac{\chi^{2}}{2}+\frac{\chi^{4}}{4(n+1)}+\frac{\chi^{6}}{3(n+1)(n+3)}+\cdots,
$$

with $\chi^{2}=\frac{1}{n(n-1)} \sum_{i=1}^{n}\left(y_{i}-\delta\right)^{2}$ being the usual estimate of the variance of the energy difference directly obtained by the sample data, and $n$ being the number of statistically uncorrelated estimates of $\delta\left(\vec{R}, \vec{R}^{\prime}\right)$. Eq.(32) must be supplemented by the condition $\chi^{2} / n \leq 1 / 4$ for the asymptotic expansion in Eq. (33) to converge.

Eq. (38) is similar to the standard Metropolis acceptance/rejection rule, with an extra rejection term $u_{B}(>0)$ which is related to the level of noise in the electronic energy difference between the two protonic configuration $R, R^{\prime}$. Since we only need to compute energy difference from QMC, we can use correlated sampling, a much more efficient method than performing two independent calculations (Ceperley et al., 2002). The efficiency of the method is sensitive to the noise level; it depends on the size of the ionic steps and on the length of the sampling of the electronic configuration space. If the noise level is too low (either because we move ions very little or we run very long electronic calculations) the extra rejections are significantly reduced but the computer time required will be very large. On the other hand, if the noise level is too high, all moves will be rejected from the penalty term. The optimal noise level depends on the temperature and is well approximated by $\chi^{2} \approx 1$.

One could get the impression that the penalty method cause a large decrease in the efficiency of CEIMC when decreasing the temperature, since the noise level in the energy would need to be at least as low as the temperature. However, at low temperatures, the nuclei need to be represented by their own path integrals and the relevant "temperature" is now the inverse of the imaginary time step $\tau$, rather then the inverse physical temperature $\beta$, and the optimal noise level is $\tau \sigma=1$ where $\sigma$ is now the noise on the energy difference at each time slice of the nuclear path. For a more detailed discussion on the implementation of nuclear path integrals in CEIMC see Pierleoni and Ceperley, 2005b.

We can improve the efficiency of the CEIMC method by using a multi-level sampling approach. Trial ionic moves are "screened" by first accepting or rejecting them using a simple effective potential, $V_{\text {eff }}(\vec{R})$ :

$$
\left.A_{1}\left(\vec{R} \rightarrow \vec{R}^{\prime}\right)=\min \left\{1, \exp \left[-\beta \Delta V_{e f f}\right)\right]\right\}
$$

where $\Delta V_{\text {eff }}=\left(V_{e f f}\left(\vec{R}^{\prime}\right)-V_{\text {eff }}(\vec{R})\right)$. If the move is accepted, the energy difference is calculated using QMC and the step accepted or rejected based on:

$$
A_{2}\left(\vec{R} \rightarrow \vec{R}^{\prime}\right)=\min \left\{1, \exp \left[-\beta\left(\Delta E_{B O}-\Delta V_{e f f}\right)-u_{B}\right]\right\}
$$

Since the evaluation of the effective potential is orders of magnitude faster than the evaluation of the QMC energy difference, the overhead produced by the pre-rejection is negligible. On the other hand, it can significantly increase the efficiency of the method by eliminating QMC calculations for "bad" steps, and increasing the effective acceptance rate.

Another promising approach for QMC-based FP simulations is that of Attaccalite and Sorella, 2008. In this approach, Langevin dynamics is used to perform a simulation with forces coming from QMC calculations. Similar to CEIMC, the forces contain a statistical uncertainty that will lead to a biased ionic sampling if used in Newtonian dynamics. Instead, the authors use a modified Langevin algorithm, robust to noise. They show that it is possible to add Gaussian correlated noise to the QMC forces, as long as the covariant matrix of the forces is finite and known. With this method, they are able to simulate liquid hydrogen close to the dissociation transition and predict a stable molecular liquid at room temperature at $300 \mathrm{GPa}$. While this calculation used VMC forces and did not include twist average boundary conditions, (see discussion on size effects below), the method shows great promise as a general purpose QMC-based first principle method for arbitrary chemical systems. Along with CEIMC, this method represents one of the frontiers in the development of next-generation (beyond DFT) firstprinciples simulation methods. ${ }^{4}$

\footnotetext{
${ }^{4}$ Note that in what is called in the literature, quantum Molecular Dynamics (QMD), classical dynamics of the ions is performed with forces computed with density functional theory.
} 


\section{E. DFT-based First Principles simulations}

Almost all first principles simulation methods using a DFT energy surface are performed with Molecular Dynamics (MD), though attempts have been reported using Monte Carlo (McGrath et al., 2006). There are two general ways to use potential energy surfaces from DFT in a MD simulation: either a fully converged calculation for the electrons is performed for every nuclear position or a unified dynamical approach is used to propagate both electrons and ions simultaneously. Both approaches are described below. Before describing the solution of the ionic problem, we make a brief description of DFT methods, which form the basis of the first-principles Molecular Dynamics (FPMD) approach. For a more detailed discussion of DFT and FPMD methods, see (Dykstra, 2005; Fiolhais et al., 2003; Hafner, 2008; Martin, 2004; Marx and Hutter, 2009; Mattsson et al., 2005; Parr and Weitao, 1994).

\section{Density Functional Theory}

Although theories based on functionals of the electron density have a long history in physics and chemistry, with the Thomas-Fermi theory as one of the earliest and better known examples (Fermi, 1927; Thomas, 1927), the term Density Functional Theory(Kohanoff, 2006; Martin, 2004; Parr and Weitao, 1994) refers to the formulation based on Hohenberg-Kohn (HK) theorems (Hohenberg and Kohn, 1964) and the Kohn-Sham (KS) ansatz $\left(\right.$ Kohn and Sham, 1965) ${ }^{5}$. The first HK theorem states that there is a one-to-one correspondence between the external potential (in this case the potential produced by the nuclei) and the ground state electronic density. This means that for every wave function that is the ground state of some Hamiltonian, the external potential giving rise to it is unique up to an additive constant. Notice that while the wave function for the many electron system lives in a $3 N_{e}$ dimensional space, the electron density is a function of only the 3 spatial coordinates. Thus, in principle, knowledge of the density implies knowledge of the wave function and, in turn, of all the properties of the system. The second HK theorem states that there exists a universal energy functional of the density, $E[n]$, defined for any external potential, such that the global minima of this functional represents the ground state energy of the system. The density at the minimum gives the ground state electronic density.

One might hope that the HK theorems could simplify the description of the many electron problem since it uses the density rather than the full wave function as the fundamental variable, but, in practice, the universal energy

\footnotetext{
${ }^{5}$ We do not describe earlier band theory methods on hydrogen since those methods have been generally superseded by DFT.
}

functional is unknown and there is currently no known accurate way of extracting properties of electronic systems from the density alone. The approach of Kohn and Sham (Kohn and Sham, 1965) was to replace the original interacting problem by an auxiliary system defined in terms of non-interacting electrons that is more tractable and easier to solve. In their formulation of DFT, which is the implementation commonly used today, the auxiliary system is defined such that its ground state electron density is the same as the density of the interacting system. This allows us to write down an explicit form for the energy functional in terms of the single body orbitals of the non-interacting system:

$$
\begin{gathered}
E_{K S}[n]=-\frac{1}{2} \sum_{i=1}^{N}\left|\vec{\nabla} \psi_{i}(\vec{r})\right|^{2}+\int d^{3} \vec{r} n(\vec{r}) V_{e x t}(\vec{r}) \\
+E_{H}[n]+E_{n n}+E_{x c}[n], \\
E_{H}[n]=\frac{1}{2} \int d^{3} \vec{r} d^{3} \vec{r} \prime \frac{n(\vec{r}) n(\vec{r} \prime)}{|\vec{r}-\vec{r}|},
\end{gathered}
$$

where $\psi_{i}(\vec{r})$ are the eigenstates of the non-interacting Hamiltonian, $E_{H}$ is the Hartree energy (the classical electrostatic interaction of the density), $E_{n n}$ is the nuclei-nuclei interaction energy, and $E_{x c}$ accounts for exchange and correlation energy. The density of the noninteracting system is defined by: $n(\vec{r})=\sum_{i=1}^{N}\left|\psi_{i}(\vec{r})\right|^{2}$.

In the KS formulation all the many body effects are encapsulated in the exchange-correlation functional, $E_{x c}[n]$, which accounts not only for exchange and correlation effects, but also for many body corrections to the kinetic energy. Although the existence and uniqueness of this functional is guaranteed by the HK theorem, its form is unknown and probably too complicated to be computed exactly. Nonetheless, it is much easier to find reasonable approximations for $E_{x c}$ since this term typically represents a small contribution to the total energy.

The ground state HK theorems were extended to finite temperature in Mermin, 1965. Although the study of the finite temperature functional has not received as much attention over the years, its independent particle formulation is used frequently in FPMD with a form, $F_{K S}[n]=E_{K S}[n]+T S[n]$, where $E_{K S}[n]$ is the usual KS energy functional. The density is a weighted average over excited states $n(\vec{r})=\sum_{i=1}^{\infty} f_{i}\left|\psi_{i}(\vec{r})\right|^{2}, f_{i}$ defines the occupation of state $i$, (e.g. using the Fermi-Dirac formula) and the entropy has the form:

$$
S=-\sum_{i} f_{i} \log f_{i}+\sum_{i}\left(1-f_{i}\right) \log \left(1-f_{i}\right)
$$

The free energy functional plays a crucial role in the study of metals and is the basis of the FEBO method discussed above. It is found that using a small but finite temperature often leads to much faster and robust convergence for certain systems at low temperatures.

The accuracy of DFT depends on the exchangecorrelation functional. The simplest possible form, 
known as the Local Density Approximation (LDA), assumes that the functional is a local function of the density:

$$
E_{x c}^{L D A}=\int d^{3} \vec{r} \epsilon_{x c}(n(\vec{r})) n(\vec{r}),
$$

where $\epsilon_{x c}(n)$ is the exchange-correlation energy density of the homogeneous electron gas with density $n$, calculated using Quantum Monte Carlo methods by Ceperley and Alder, 1980 and subsequently parameterized by Perdew and Zunger, 1981. While the LDA generally produces reasonable results, in particular for weakly inhomogeneous systems, current calculations typically include information about the density variation, producing better approximations. In the next level of accuracy, the Generalized Gradient Approximation (GGA), a semi-local expansion of the exchange-correlation density is used including also the density gradient, typically expressed in terms of the dimensionless combination $s=|\vec{\nabla} n| /\left(2 k_{F} n\right)$. There are various implementations of GGA functionals, e.g. variational forms fitted to reproduce experimental results in molecular systems and condensed phases, and implementations based on perturbation treatments. The Perdew-Burke-Enzerhof (PBE) (Perdew et al., 1996a) exchange-correlation functional is the most used choice for dense hydrogen, and, as we will show, produces a good description at high pressure.

Beyond the GGA approximation, in the metaGGA formulation (Perdew and Schmidt, 2001) the non-interacting kinetic energy density, $\tau(\vec{r})=1 / 2 \sum_{i}\left|\vec{\nabla} \psi_{i}(\vec{r})\right|^{2}$, is used in the construction of the exchange-correlation energy density. In the orbital dependent formulations(Kümmel and Kronik, 2008), the Kohn-Sham orbitals are used in the construction of the exchange-correlation functional. In this case, a generalized KS formulation must be used because the resulting potential becomes orbital dependent; this invalidates the original formulation by HK. Nonetheless, the use of orbitals still allows for a rigorous formulation of DFT and, in general, produces a large increase in accuracy and has become the standard for quantum chemistry calculations. The best example of orbital dependent DFT is the hybrid functional approach, where the exchange-correlation functional includes a fraction of exact exchange from Hartree-Fock theory (Becke, 1993). The best known hybrid functionals in the condensed matter community are PBE0 (Perdew et al., 1996b), which uses a mixture of $25 \%$ of Hartree-Fock exchange with $75 \% \mathrm{PBE}$, and the HSE functional (Heyd et al., 2003a), which uses a combination of range separation and the same mixing fractions as in PBE0; the Hartree-Fock calculation is done only on the short range part of the potential.

The KS band gap differs from the true one by a discontinuity in the derivative of the exchange-correlation potential $\nabla_{\mathrm{xc}}$ (Perdew and Levy, 1983) with respect to density. Since $\nabla_{\mathrm{xc}}=0$ for standard DFs, the width of the gap at a given density (pressure) as well as the density at which it actually closes are both underestimated (Johnson and Ashcroft, 2000; Städele and Martin, 2000). However, the exact exchange method (Städele et al., 1997, 1999) provides an approximation to $\nabla_{\mathrm{xc}}=0$; the resulting nonlocality in exchange causes an overestimation of the width of the gap. One can partially cancel these errors by mixing a fraction of exact exchange with a standard DF, such as is done by Heyd-ScuseriaErnzerhof (HSE) (Heyd et al., 2003b): relatively accurate band gaps are obtained (Brothers et al., 2008). Exact exchange functionals, e.g. HSE, require significantly more computational effort compared to a standard DF. An alternative to hybrid functionals are many-body Green's function techniques, e.g. the "GW" approximation (Fiolhais et al., 2003; Onida et al., 2002), which has been applied to hydrogen with encouraging results (Chacham and Louie, 1991; Chacham et al., 1992).

In another problem, most DFs (i.e., those which treat electron correlations at a local or semilocal level) cannot describe vdW interactions ( dispersion interactions), giving rise to errors in the application of DFT to the low-pressure region of the hydrogen phase diagram, where weak vdW interactions between molecules are very important (Silvera, 1980). Recent progress in DFT has been made, however, with the advent of socalled vdW DFs (Dion et al., 2004; Lee et al., 2010) and semi-empirical density-dependent vdW corrections (Tkatchenko and Scheffler, 2009).

Once an approximate exchange-correlation functional is chosen, the DFT energy functional is well defined. For calculations, we must first choose the representation of the orbitals, $i$. e. the basis set. The most common bases are plane waves and linear combinations of localized functions, e.g. Gaussian functions. For calculations with periodic boundary conditions, plane waves are natural since they represent extended, delocalized states and they do not suffer from superposition errors of localized bases: the orbitals are written as:

$$
\psi_{i}(\vec{r})=\sum_{\vec{G}} c_{\vec{G}}^{i} e^{i \vec{G} \cdot \vec{r}}
$$

where $\vec{G}$ belong to the reciprocal space of the simulation cell and the sum extends over all vectors such that $|\vec{G}|^{2} / 2<\mathrm{E}_{\text {cut }} . \quad \mathrm{E}_{\text {cut }}$ is a cutoff parameter that controls the accuracy of the expansion. The value of the $\mathrm{E}_{c u t}$ needed to achieve a given accuracy depends on details such as the pseudopotential (see below), and the nature of the electronic states. While the kinetic and Coulomb energies are given by sums over $(\vec{G}$, the exchange-correlation energy is most easily evaluated as a sum over a real space grid. The Fast Fourier Transform method is used to transform between real and reciprocal space during the solution.

However, plane waves require a dense grid to represent localized or highly oscillatory orbitals. This creates a problem for core states which localized in the core region. In the majority of systems, core states are chemically inert so we should be able to remove them from 
the calculation without affecting the chemical properties which are determined by the valence states. Pseudopotentials are renormalized electron-nuclei potentials for the valence states that include both the Coulomb attraction of the nuclei as well as the screening effects resulting from the presence of core electrons. By employing pseudopotentials, not only do we remove core states from the calculation, but we also obtain valence states which are smooth in the core region; this greatly reduces the computational demands. There are many different types of pseudopotentials with different levels of transferability (the ability to reproduce the properties of the atom under different environments) and complexity. For additional details see Martin, 2004.

In DFT calculations, pseudopotentials are used for both hydrogen and helium even though neither of them possesses core electrons, but because the Coulomb potential, $1 / r$, requires a large plane wave cutoff greatly increasing the computational demands of the calculations. Since the pseudopotentials are built to reproduce the scattering properties of the atom, valence states should not be significantly affected outside the core region.

\section{Treatment of proton zero point motion}

Within the normal framework of DFT, the treatment of nuclear quantum effects poses challenges. These are especially important in hydrogen, due to the light proton mass. Often, the treatment of ZPM is perturbative, adding corrections to the ground-state DFT results. Perhaps the simplest approximation is obtained by considering the ZPM to be harmonic, by neglecting anharmonic terms. The harmonic approximation gives the zero-point energy ZPE as an integral over the phonon density of states $F(\omega)$ :

$$
E_{\mathrm{ZPE}}=\int d \omega F(\omega) \hbar \omega / 2
$$

While this approximation is widely employed, it has long been known that this approximation can easily fail for light elements. For example, early calculations by Straus and Ashcroft, 1977 showed that the harmonic approximation can incorrectly predict the relative stabilities of atomic crystal structures (see Section IV.A.6). In particular, only by treating coupling between the phonons self-consistently (i.e., including anharmonicity) are some structures energetically stabilized. In fact, anharmonic terms can be roughly equal in magnitude to the harmonic ones (Natoli et al., 1993). While methods for approximating anharmonicity in ZPE estimates, such as the selfconsistent $a b$ initio lattice dynamics method (Souvatzis et al., 2008) exist, these are still approximate, considering protons as classical particles. Rigorous inclusion of proton ZPM can be obtained by QMC calculations treating both the nuclei and electrons quantum mechanically at $T=0 \mathrm{~K}$ (Ceperley and Alder, 1987a; Natoli et al.,
1993) or by using Path Integral methods at a sufficiently low temperature as discussed elsewhere in this section.

\section{Born-Oppenheimer Molecular Dynamics}

The simplest, and more computationally time consuming way to perform FPMD simulations with DFT is to evaluate the forces on the nuclei at each MD step from a fully converged DFT calculation. This approach is known as Born-Oppenheimer Molecular Dynamics (BOMD) (Marx and Hutter, 2009; Payne et al., 1992). The forces on the ions are calculated by the Hellman-Feynman theorem (Feynman, 1939; Hellmann, 1937), valid for the orbitals and density that minimize the KS energy functional:

$$
\begin{aligned}
\vec{F}_{i}=-\vec{\nabla}_{i} E_{B O}^{K S} & =-\vec{\nabla}_{i} \min _{\left\{\phi_{k}\right\}} E_{K S}[\phi] \\
& =-\left\langle\left\{\phi_{k}\right\}\left|\vec{\nabla}_{i} \hat{H}_{K S}\right|\left\{\phi_{k}\right\}\right\rangle .
\end{aligned}
$$

Since the Hellmann-Feynman theorem is only applicable at the variational minimum of the KS energy functional, the DFT calculation must be well converged. This makes the approach more expensive than the alternative method described below. Almost all DFT calculations employ iterative schemes to minimize the KS energy functional. The number of iterations needed to reach convergence depends crucially on the initial guess for the orbitals and electronic density. In order to make the calculations fast enough to make the BOMD approach practical for large systems, the orbitals at time $t+d t$ are estimated from the results at times $(t, t-d t, t-2 d t, \ldots)$, thus saving large factors of computer time, especially for systems with many electrons.

In the case of Free Energy Born-Oppenheimer simulations, which includes thermal electronic excitations, the forces are calculated as the gradients of the electronic free energy functional, $\vec{F}_{i}=-\vec{\nabla}_{i} F_{B O}^{K S}$, corresponding to the approximation described in section II.B .

\section{Car-Parrinello Molecular Dynamics}

The Car-Parrinello Molecular Dynamics method (CPMD) (Car and Parrinello, 1985; Vuilleumier, 2006) gave birth to the field of first-principles simulations; it represented a major breakthrough in our ability to use computers to study the properties of materials. The approach enabled the direct study of the thermodynamic, optical and transport properties of materials using much more realistic interatomic forces since the forces were calculated "on the fly" using DFT. CPMD is the main alternative to BOMD, appropriate if there is a gap in the excitation spectrum of the electrons. In the CPMD approach, instead of treating the electron and nuclear problems independently, the dynamics of both the ions and the electronic KS orbitals are done simultaneously, but still within the the BO approximation. The Lagrangian, 
used to define the dynamics, depends on the potential energy surface, regarded as a function, not only of the nuclear positions, but also of the electronic degrees of freedom: $E^{K S}=E^{K S}\left(\vec{R},\left\{\Psi_{i}\right\}\right)$. The orthogonality between KS orbitals is enforced using Lagrangian multipliers. The resulting Lagrangian is given by:

$$
\begin{aligned}
\mathcal{L}= & \sum_{i=1}^{N_{e}} \mu \int d \vec{r}\left|\dot{\Psi}_{i}(\vec{r})\right|^{2}+\sum_{I=1}^{N_{n}} \frac{1}{2} M_{I} \dot{\vec{R}}_{I}^{2}-E^{K S}(\Psi, \vec{R}) \\
& +\sum_{i, j} \Lambda_{i j}\left[<\Psi_{i} \mid \Psi_{j}>-\delta_{i j}\right]
\end{aligned}
$$

where $\mu$ is a fictitious mass assigned to the electrons. This leads to a set of equations of motion for the orbitals and nuclear positions:

$$
\begin{aligned}
\mu \ddot{\Psi}_{i}(\vec{r}, t) & =-\hat{H}_{K S} \Psi_{i}(\vec{r})+\sum_{k} \Lambda_{i k} \Psi_{k}(\vec{r}, t), \\
M_{I} \ddot{\vec{R}}_{I} & =\vec{F}_{I}^{K S}=-\frac{\partial E^{K S}}{\partial \vec{R}_{I}} .
\end{aligned}
$$

In addition, thermostats and barostats are added to produce constant temperature or variable cell algorithms (Hutter et al., 1995; Martyna et al., 1994; Tuckerman and Parrinello, 1994a,b)

A simulation is begun using orbitals that minimize the KS energy functional. During the simulation, both nuclear positions and electronic orbitals are evolved in time simultaneously. The fictitious mass is adjusted so that the electronic sub-system remains sufficiently close to the Born-Oppenheimer energy surface, while the nuclei are kept at the physical temperature, $T$. In a successful application of the method, the electronic degrees of freedom oscillate around the instantaneous BO energy surface throughout the entire simulation. This can only be achieved if the flow of energy between the electronic and nuclear degrees of freedom is eliminated, or reduced to a point where long enough simulations are possible before heating effects of the electronic degrees of freedom are seen. For the orbitals to follow the nuclei adiabatically and energy transfer not take place, the power spectra in the frequency domain of the two subsystems should not overlap (Marx and Hutter, 2000). This can be achieved for a system with an electronic gap and for a careful choice of the (fictitious) mass of the electronic orbitals. But for metals, the flow of energy between the two sub-systems is hard to control. In practice, a separate thermostat can be applied to the electronic degrees of freedom to maintain them at low temperature. With the development of faster and more robust algorithms for DFT calculations, BOMD simulations have slowly gained popularity over CPMD, since they are easier to control and offer smaller chances of failure compared to CPMD. Nonetheless, CPMD is still a widely used and applicable method for FPMD simulations.

\section{Path Integral Molecular Dynamics}

As mentioned in subsection II.B, it is possible to introduce nuclear quantum effects within a $\mathrm{BO}$ framework with Path Integral methods. The ionic quantum problem at finite temperature is mapped to a classical problem where each ion is represented by a ring polymer system; see II.B. If DFT is used to determine the forces arising from the electrons with either of the methods presented above, (CPMD or BO), the usual approach is to simulate ionic degrees of freedom (the ring polymers) also with molecular dynamics: Path Integral Molecular Dynamics (PIMD). To achieve adequate sampling of the phase space of the ionic polymers, well designed thermostats need to be used (Marx and Parrinello, 1996; Tuckerman et al., 1993). Recent developments by Ceriotti M., et al. (Ceriotti et al., 2011, 2010) represent a very promising alternative to thermalize PIMD simulations efficiently using generalized Langevin equations. Note that much more computer time is needed to include quantum effects of the nuclei, typically by one to two orders of magnitude, depending on the temperature and mass of the nuclei.

\section{F. Size Effects}

No discussion of simulation methods is complete without discussing the problem of size effects. Simulation methods try to reproduce the properties of bulk materials by studying a finite number of atoms in periodic boundary conditions. Since the particles interact with their (artificial) periodic images, the resulting calculations depend on the number of atoms used in the primitive cell. In principle, the most straight forward way to eliminate these effects is to study larger systems until the computed properties are independent of the number of atoms. While this is possible in simulations with semi-empirical potentials, first principles simulations are limited to systems of up to $\sim 1000$ electrons. Hence, great care must be taken to ensure that results are close to the thermodynamic limit.

Size effects come in various forms and affect results in different ways; they can be divided into electronic and structural origin. Electronic size effects come about because electronic wavefunctions can be sensitive to the size and shape of the simulation cell and the coulomb interaction between particles that are far apart must be correctly taken into account. This is particularly important in metallic systems where electronic states are delocalized over extended regions of the material. In electronic structure methods based on the single body picture like DFT, size effects are handled by integrating over the Brillouin zone of the unit cell, often called k-point integration (Martin, 2004). In this case it is possible to remove all electronic size effects from a unit cell of arbitrary size by considering sufficiently dense grids in the Brillouin zone; smaller unit cells require denser grids. In calculations at the so-called $\Gamma$-point Brillouin zone integration is not 
done, and are thus susceptible to finite size errors. For all many-body methods such as QMC and PIMC there is, in principle, no way to obtain fully converged results using small unit cells exclusively; it is always necessary to consider progressively larger unit cells until results can be safely extrapolated to the infinite cell limit.

The understanding of size effects in many body systems has progressed considerably over the last decade. The first step came with the introduction of twist averages boundary conditions (TABC) in QMC simulations (Lin et al., 2001). TABC is the generalization of Brillouin zone integration to many-body quantum systems and is used to eliminate shell effects in the kinetic energy of metallic systems. By twisted boundary conditions, we mean:

$$
\Psi_{\theta}\left(\vec{r}_{1}, \ldots, \overrightarrow{r_{j}}+\vec{L}, \ldots, \vec{r}_{N}\right)=e^{i \theta} \Psi_{\theta}\left(\vec{r}_{1}, \ldots, \vec{r}_{j}, \ldots, \vec{r}_{N}\right)
$$

where $\Psi_{\theta}$ is the many-body wave function of the system and $\vec{L}$ is the size of the periodic cell. Observables are then averaged over the all twist vectors, similar to the procedure in single-body theories:

$$
\langle\hat{A}\rangle=\int_{-\pi}^{\pi} \frac{d \vec{\theta}}{(2 \pi)^{3}}\left\langle\Psi_{\theta}|\hat{A}| \Psi_{\theta}\right\rangle .
$$

In Chiesa et al., 2006 it was shown that most of the remaining finite size errors in the energy come from discretization errors induced by the use of $\mathrm{PBC}$, in particular in the incorrect treatment of charge-charge interactions at wavelengths greater than the simulation cell. This work lead to explicit formulae for the finite size corrections to the energy and the pressure that are quite accurate in practice (Chiesa et al., 2006; Drummond et al., 2008). The method has been extended to the Fermi-liquid parameters in the homogeneous electron gas (Holzmann et al., 2011), and the renormalization factor of sodium (Huotari et al., 2010).

Size effects can also affect structural properties of the ions as the following two examples illustrate. For a finite simulation cell, only density fluctuations smaller than the length of the simulation are non-zero. This can be important near a phase transitions, in particular, near critical points in the phase diagram, since there fluctuations have a longer wavelength. Secondly, in simulations of solids, only structures commensurate with the chosen simulation cell can be reached in a simulation causing a possible bias in structural predictions.

\section{G. Other Theoretical Methods}

Here we briefly review other theoretical methods applied to hydrogen and helium at high pressure, focusing on the type of approximations employed and their expected range of applicability. With the rapid development and widespread use of first-principles methods over the last two decades, the use of semi-empirical methods has become less important. Nonetheless, they can produce reliable and accurate results, and can be combined with first principles methods, for example, by using FPMD results to determine intermolecular forces (Ercolessi and Adams, 1994).

\section{One Component and Screened Coulomb Plasma Models}

For temperatures and densities where the atoms are fully ionized, the electrons can be integrated out, and the proton-proton pair interaction treated using linear response theory. In the limit of very high density, the electrons behave as a rigid background and the system can be modeled as the One Component Plasma. This model, with classical protons, has been extensively studied since the early days of simulation (Brush et al., 1966) to compute thermodynamics, structural and dynamical properties (Hansen, 1973; Hansen et al., 1975; Pollock and Hansen, 1973; Vieillefosse and Hansen, 1975). The effect of quantum zero point motion on the melting line has also been studied(Jones and Ceperley, 1996) by Path Integral Monte Carlo.

At lower densities, the electrons cannot be treated as a uniform, non-responding background. However, if the density is high enough $\left(r_{s}<0.6\right)$ for the electron-proton coupling to be a small perturbation to the electron system, the electronic screening can be computed by linear response theory, and the electron-proton system mapped onto a system of protons interacting through an effective pair potential; the bare Coulomb potential screened by the electrons: the Screened Coulomb Plasma model (Ashcroft and Stroud, 1978; Galam and Hansen, 1976; Hansen and McDonald, 1981). This model has been studied in the 1970's by Monte Carlo and thermodynamic perturbation methods in conditions of high temperature (low coulomb coupling) relevant to stellar interiors (Dharmawardana and Perrot, 2002; Hubbard, 1972; Hubbard and Slattery, 1971; Ichimaru, 1982; Ross and Seale, 1974; Totsuji and Tokami, 1984), and, recently, at much lower temperature to compute the melting line (Liberatore et al., 2011b) of metallic hydrogen and deuterium at ultra-high pressure. The electronic response function, the property of the many body electron system that determines the effective proton's interaction, has been the subject of many investigations: many-body techniques, perturbation theory and diagrammatic theory, and numerical work using DFT and QMC (Giuliani and Vignale, 2005). Note that these are ab-initio models, however, the range of validity of the description in terms of an effective interaction is limited to very high density or pressure. At lower density, non-linear response theory needs be used and the interaction between protons is no longer simply a pair potential, complicating the description as an effective interaction(Nagao et al., 2003). 


\section{Semi-empirical Methods and Chemical Models}

At low density, hydrogen forms molecules and an accurate description can be obtained by semi-empirical models. At densities where the bond length of $\mathrm{H}_{2}$ is less than the distance between molecules, it is accurate to assume that $\mathrm{H}_{2}$ is a spherical particle interacting with other molecules through pair potentials, in particular for parahydrogen and ortho-deuterium. The effective potential has been optimized to represent the EOS of solid and liquid hydrogen at low pressure by Silvera and Goldman (SG) (Silvera and Goldman, 1978). The SG potential reproduces the fluid isotherms of hydrogen from 75 to $300 \mathrm{~K}$ up to $2.0 \mathrm{GPa}$ and the melting curve to $5.7 \mathrm{GPa}$, but can not predict the Hugoniot curves. To remedy this Ross et al., 1983 proposed a modified effective potential, the Ross, Ree, and Young (RRY) potential, by softening the SG potential at short range. Further improvements to match experimental data at higher pressures and temperatures in the liquid phase have been proposed by Ross, Ree and Young and by Hemley, and tested in Matsuishi et al., 2003. Unfortunately, the transferability of effective potentials (i.e. the ability of a given potential fit in one set of conditions to adequately represent data for different conditions) is quite limited even when the system remains in the same thermodynamic phase.

More difficult is to extend the semi-empirical approach to higher temperatures and/or higher densities under conditions where hydrogen is changing from molecular, to atomic and, ultimately, to the plasma state. Both pressure and temperature play roles in the transformation. In order to model this complex system, free energy models based on the chemical picture have been developed. One assumes a fixed set of chemical species, using pair interactions typically taken from empirical data, calculations or from perturbation theory. The free energy of the system is typically calculated assuming that the partition function factorizes into internal (vibrational-rotational and electronic excitations) and external (interaction between centers of mass) degrees of freedom. The resulting model is solved using some form of thermodynamic perturbation theory, integral equations from the theory of liquids or classical simulations.

Several chemical models for hydrogen and hydrogenhelium mixtures have been proposed in the astrophysical community over the last 40 years (Ebeling and Richert, 1985; Juranek and Redmer, 2000; Juranek et al., 2003; Ross et al., 1983; Saumon et al., 1995). An elaborate chemical model, known as the SCVH model, has been developed in Saumon et al., 1995 by assuming a mixture of $\mathrm{H}_{2}, \mathrm{H}, \mathrm{e}^{-}$and $\mathrm{p}^{+}$and suitable pair potentials. This model reproduces most of the experimental data available, both at low temperature/high pressure (before crystallization) and high temperature/high pressure. The hydrogen EOS provided by this model is a standard for the planetary physics community. Another well known model for hydrogen was developed in Kerley, 1972, 2003a. Its latest version produces results in very good agreement with first-principles simulations after a simple correction is made (Morales et al., 2012). A similar strategy has been developed to model high pressure helium and to predict its EOS in Winisdoerfer and Chabrier, 2005; however, existing models for $\mathrm{H}-\mathrm{He}$ mixtures are still based on the EOS for the two pure systems and the linear mixing assumption. We show in Section V.B the limitations of this assumption.

\section{H. Comparison of Simulation Methods}

To conclude our discussion of computational methods for dense hydrogen and helium we briefly summarize some of the strengths and weaknesses of two most well developed simulation methods: methods based on DFT and those based on QMC. Both methods are reliable $a b$ initio methods and have been made feasible by the computer hardware available today.

Although DFT is, in principle, an exact theory, in practice only approximations to the density functional (DF) are known. This results in a number of challenges associated with the application of DFT to the study of dense hydrogen and helium. At present, many DFs exist(Burke, 2012). Moreover, it is not a priori known which is the most accurate for a given problem without experimental data or explicitly correlated calculations (e.g., QMC) to compare to. In other words, for any given problem, there is no internal measure of error within DFT with respect to the choice of the DF.

DFT in general shows serious deficiencies describing non-equilibrium geometries, such as reaction barriers and configurations with competing bonding patterns (Foulkes et al., 2001; Martin, 2004). The simplest example, also particularly pertinent to our focus, is the molecular-toatomic transition in hydrogen, $\mathrm{H}_{2} \rightarrow 2 \mathrm{H}$ as two protons are pulled apart. The quality of DFT description of each state $\left(\mathrm{H}_{2}\right.$ and $\left.2 \mathrm{H}\right)$ is likely not equivalent, and so the physics associated with molecular dissociation will be poorly described. A very much related problem, discussed already in Section II.D.1 is the accurate description of the band gap. Clearly one needs a reliable method for the band gap to describe well the metal-insulator transition in hydrogen.

QMC methods, are able to get around some of the problems that DFT methods have. For hydrogen, perhaps the biggest advantage is that QMC is able to treat molecular dissociation rather simply, while DFT methods have difficulty. In addition, no assumption is made about the density functional, and QMC does not need either pseudopotentials or basis sets. Another advantage of QMC methods is their ability to treat the zero point motion of the protons in the same formalism, i.e. without making the Born-Oppenheimer approximation: at zero temperature by using projector methods for both electrons and nuclei, or using RPIMC for temperatures at non-zero temperatures.

However, QMC methods typically take more computer 
resources than DFT methods and they come with different types of errors, both statistical and systematic. QMC methods face a difficulty arising from the mass difference of the proton and electron: since $M_{p} / m_{e} \simeq 1836$, the electrons move much faster than the protons in the DMC dynamics, resulting in a very significant computational cost. At finite temperature, the mass ratio requires electronic paths to be much longer than nuclear paths causing difficulty in sampling the path space. The major conceptual bottleneck in QMC calculations is the "fermion sign problem": a direct QMC calculation is very inefficient; one has to resort to the fixed node or fixed-phase approximation for calculations of extended systems, requiring an ansatz for when the wavefunction or density matrix changes sign. In most cases, fixed-node QMC methods have proved to be much more accurate than mean field methods. In contrast to DFT, the variational principle provides a rigorous way of deciding which ansatz is superior. RPIMC remains the only method to test(Militzer and Ceperley, 2001) predictions of chemical models(Saumon et al., 1995) in the low density region of the phase diagram $(P \leq 1 G p a)$ where molecules are well formed and nuclear motion occurs in the electronic ground state. In this situation the detailed structure of the nodes become irrelevant since electrons are paired in the bonding singlet state inside each molecule while molecules interact with the dipole-induced-dipole mechanism. Conventional DFT methods will be inaccurate and also very expensive unless localized basis sets are used.

In the past, QMC simulations have been limited in their ability to do simulations of sufficiently large systems. However, because QMC can be more easily adapted to a highly parallel environment, the computational limitations have lessened. As we will show below, DFT and QMC, in fact, are giving similar results in many cases, providing confidence in their predictions.

\section{EXPERIMENTAL METHODS}

The two most important experimental methods that have been used in the last decades to study hydrogen at high pressures, are dynamic compression methods (Nellis, 2006b) based on shock experiments and static compression methods (Goncharov and Hemley, 2006; Mao and Hemley, 1994) based on diamond anvil cells. These two complementary techniques are applicable in different pressure and temperature conditions, as discussed in Section I. In this section, we outline these methods, in order to understand the results that follow.

\section{A. Dynamical Compression}

Dynamical compression techniques apply a very strong force to a small sample and then measure the properties of the resulting shockwave and its aftermath with special- ized techniques. These methods can achieve higher pressures and temperatures than static methods, for example $\sim 500 \mathrm{GPa}$ in $\mathrm{H}_{2}$ and temperatures of $\sim 50000 \mathrm{~K}$. However, the measurements typically have large uncertainties (e.g., in the resulting pressures and temperatures, or in the measured properties) since the measurements must be done very rapidly. In addition, there are limitations in the accessible values of pressure and temperature, as discussed below.

Traditional dynamical methods apply one or more shocks to a system, producing a sharp increase in pressure within a short time (e.g., $100 \mathrm{GPa}$ over $1 \mathrm{ps}$ ). A number of techniques have been developed to produce shocks, including gas guns (Nellis et al., 1983a), laser-driven compression (Collins et al., 1998a,b; Da Silva et al., 1997; Hicks et al., 2009; Sano et al., 2011b), magnetically driven flyers (Knudson and Desjarlais, 2009; Knudson et al., 2001, 2004), and hemi-spherically converging explosives (Boriskov et al., 2005). Because the shock process is adiabatic, large increases in temperature also occur with the increases in pressure.

If the applied force is strong enough, a shock wave propagates into the sample. One measures the velocity of the material at the surface of the sample and that of the resulting shock wave. Assuming the shock is a plane, the conservation laws of mass, momentum and energy lead to the Rankine-Hugoniot equations. In principle, this gives the pressure, energy and density of the postshocked material in terms of the measured velocities and the values of the initial energy, pressure, and density. The locus of such states reachable from a given initial state using different amounts of applied force is called the Hugoniot curve, and satisfies the equation (Zeldovich and Raizer, 1967):

$$
E-E_{0}=\frac{p+p_{0}}{2}\left(v_{0}-v\right)
$$

where $E, p, v$ are specific internal energy, pressure and specific volume, respectively and the subscript 0 refers to the state of the sample prior to the shock. One can explore different regions in phase space, by shocking precompressed samples, or measuring properties after the shock wave reflects from a boundary, the so-called double shock. However, shock compression cannot access all values of temperature and pressure.

Hugoniot states for $\mathrm{D}_{2}$ up to $\sim 100 \mathrm{GPa}$ achieved by gas-guns (Nellis et al., 1983a), pulsed laser (Da Silva et al., 1997), magnetically-driven flyers (Knudson and Desjarlais, 2009), and hemispherically converging explosives (Boriskov et al., 2005) are shown in Fig. 1. Many shock measurements have been performed on deuterium rather than hydrogen, because the initial density of deuterium is higher (its larger mass reduces the zero point motion, the molecular bond length and the spacing between molecules) and has a lower shock impedance. Therefore, the same shock applied to hydrogen and deuterium will achieve higher pressures in deuterium. The principle Hugoniot for hydrogen has recently been measured up to 55 GPa using laser-driven shock compression 
(Sano et al., 2011b). See section IV.B.1 for a detailed discussion of the measured and calculated Hugoniots of $\mathrm{H}_{2}$ and $\mathrm{D}_{2}$.

More complex dynamical methods exist to reach offHugoniot states; the simplest is a double-shock compression (Fortov et al., 2003b; Nellis et al., 1983a). Doubleshock data for $\mathrm{D}_{2}$ (Nellis et al., 1983a) is also shown in Fig. 1. Other methods include explosive-driven generators (Fortov et al., 2007), as well as shock reverberation (Weir et al., 1996) and isentropic compression (Nellis, 2006b). The latter methods are capable of achieving lower temperatures than in a single shock, as the compression occurs over much longer timescales. In fact, dynamic pressures and temperatures can be tuned independently between states on the Hugoniot and those obtained by isentropic compression, allowing many $(P$, $T$ ) points to be reached. However, because of their complexity, it is more difficult to assess errors of the measurements.

If the data obtainable from dynamical measurements were highly accurate, besides being useful for planetary modeling, they would constitute an excellent test of simulation methods. However, the shock velocities need to be measured very precisely and accurately to get reliable EOS points. Furthermore, there are many assumptions in analyzing the measurements. For a single shock compression, one assumes that the material in front of the shock is not preheated, that the material after the shock passes through is in equilibrium, that the shock remains one dimensional and parallel to the camera angle, and that there are no other shock waves reflected from the boundaries of the sample. Note that the temperature is not directly measured but is usually estimated by fitting the spectrum of the emitted radiation to a grey-body formula. Hence, a further assumption is that the radiation is predominately coming from the material after it has been shocked and not from other parts of the apparatus.

There are advantages and disadvantages to dynamical compression techniques; while shock compression can reach relatively high pressures and temperatures, at the same time, the conditions are transient (the time of the shock), reaching general $(P, T)$ is difficult, and there are large uncertainties in the measured properties.

\section{B. Static Compression}

Static compression techniques, produced by diamond anvil cells can realize extreme $(P, T)$ conditions (Goncharov and Hemley, 2006; Mao and Hemley, 1994). Because those conditions are static or nearly so, they are more precise and accurate than the dynamical experiments but the pressures and temperatures are also more limited. Recent advancements allow pressures near the limit of mechanical strength of the diamond anvil cells (DACs), $320 \mathrm{GPa}$ in hydrogen (Goncharov et al., 2001; Loubeyre et al., 2002); temperatures of thousands of $\mathrm{K}$ can also be obtained, for example $1500 \mathrm{~K}$ at $140 \mathrm{GPa}$ was recently obtained in fluid $\mathrm{H}_{2}$ (Subramanian et al., 2011) by heating of a small portion of the sample. The range of these conditions is outlined in Fig. 1.

In general, static compression methods are based on compressing a sample between diamond anvils (used for their large mechanical strength) and heating it using external resistive couplers or lasers. Metal gaskets are placed along the sides of the system to prevent the escape of the sample. Optical measurements, Raman or x-ray scattering can be used to monitor the sample.

At high pressures, the chemical reactivity of a material often greatly increases, and hydrogen is no exception. This causes challenges, as chemical reactions can occur with both the gasket material and the diamond anvils. The reaction with the gasket can contaminate the sample. Also hydrogen can penetrate into small cracks and escape, or catalyze the growth of fractures in the diamond. Because hydrogen is so compressible, stresses in the diamond anvil are more of a problem than with other samples, and limit achievable pressures to lower values than in less compressible materials. However, the recent advancement of using liners, such as gold (Datchi et al., 2000), have allowed pressures near the limit of mechanical strength of the diamond anvils $\sim 320 \mathrm{GPa}$ (Goncharov et al., 2001; Loubeyre et al., 2002).

Creating high temperatures has also been a challenge for static methods since the diamonds become less stable. Two types of heating methods exist, external resistive heating and laser heating. External heating provides uniform temperatures, but limits accessible temperatures to less than $\sim 1000 \mathrm{~K}$. Laser heating of a small part of the sample is capable of producing higher temperatures, but can result in large thermal gradients. However, recent advancements now allow relatively uniform heating (Lin et al., 2004); temperatures up to $1500 \mathrm{~K}$ have been achieved in $\mathrm{H}_{2}$ (Subramanian et al., 2011).

Measuring the precise pressures and temperatures that are achieved is challenging. Pressure sensors often rely on the optical properties of materials, such as the fluorescent properties of small ruby crystals embedded in the sample. Their signals decrease and broaden with temperature. Both temperature and pressure sensors only measure local conditions and their gradients may be important. There are additional challenges associated with the temperatures affecting pressures, drift of the diamond anvil cells, etc. However, many of these have been minimized to a large extent, as outlined in Goncharov and Hemley, 2006, allowing relatively precise $(P, T)$ points to be studied.

The major advantage of static compression methods is that they allow long, accurate, and repeatable measurements at precise thermodynamic $(P, T)$ conditions. These include optical properties, such as IR and Raman spectra (Goncharov et al., 2001; Loubeyre et al., 2002), and structural properties using X-ray diffraction (Akahama et al., 2010) though the low electron density of hydrogen makes the signal very weak. These measurements have been vital for understanding the low-temperature 
properties of hydrogen, as we will discuss in Section IV.

\section{Coupling Static and Dynamic Compressions}

Recently, very promising techniques that combine static and dynamic compressions hold promise to explore the phase diagram of hydrogen and other systems over a broader range of conditions. Since the Hugoniot is entirely determined by the initial state of the sample, precompression of the sample allows one to explore a range of final states. The feasibility of these experiments was demonstrated on hydrogen. Loubeyre et al., 2004, for example, used DACs to precompress hydrogen, which was then shocked using a focused laser. This demonstration provided consistent results for thermodynamical points between the principal Hugoniot of cryogenic hydrogen subjected to a single shock to those generated by a reverberating shock wave experiment (Weir et al., 1996). In another study (Grishechkin et al., 2004b) precompressed gaseous targets were shocked. The same technique has been further applied to other systems, such as high pressure helium and water (Eggert et al., 2008a; Jeanloz et al., 2007).

\section{HYDROGEN UNDER EXTREME CONDITIONS}

In this section, we discuss the properties of pure hydrogen under extreme conditions. Because experimental studies under such conditions pose many challenges, much of our understanding comes from theoretical predictions and simulations. Theoretical and computational efforts to understand the low-temperature properties are first discussed, namely, the solid phases. Properties of the fluid phase follow, with a particular focus on the liquid-liquid phase transition. A discussion of some novel theoretically-predicted phases including superconducting and low- or zero-temperature liquid states, concludes this section.

\section{A. Solid Phases}

Experimental measurements have revealed the existence of at least three low-temperature solid phases, with the possibility of two more at elevated temperatures. What is currently known about the phase diagram from experiments and simulations is shown in Fig. 3; we discuss this below. Theoretical calculations have greatly contributed to its understanding, predicting the existence and qualitative properties prior to experimental discovery of several phases. In this subsection, we first discuss the low-temperature (ground-state) solid phases as well as those at elevated temperatures that have been experimentally observed, but where much less is known. Following this, we discuss theoretical and computational predictions of the existence of additional phases at pressures beyond those currently experimentally accessible.

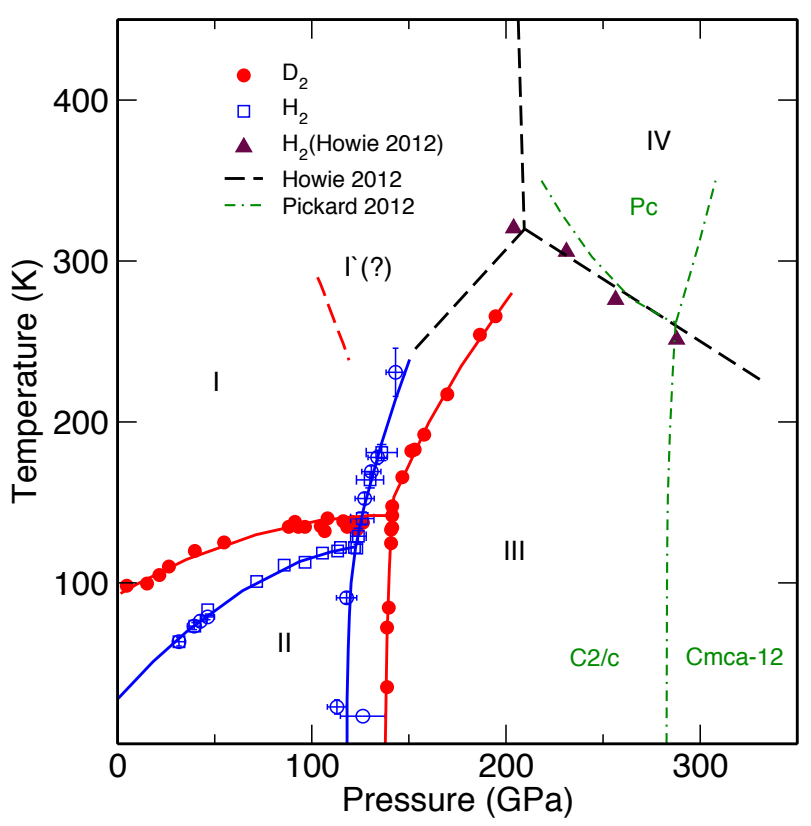

FIG. 3 (Color online) High-pressure phase diagrams of solid molecular $\mathrm{H}_{2}$ and $\mathrm{D}_{2}$, adapted from Goncharov et al., 2011. Open and filled circles are Raman measurements for $\mathrm{H}_{2}$ and $\mathrm{D}_{2}$, respectively, and open squares are IR data for $\mathrm{H}_{2}$. Brown triangles are recent experimental data on $\mathrm{H}_{2}$, and the black dashed lines indicates inferred boundaries for the recently proposed phase IV at elevated temperatures (Howie et al., 2012). Green dot-dashed lines indicates boundaries for high pressure phases obtained by AIRSS and quasi-harmonic methods (Pickard et al., 2012). Note that the dotted line and the existence of phase I' are not certain and are still matters of debate (Goncharov et al., 2011).

Finally in this section, we consider metallization and molecular dissociation to an atomic state, effects that may or may not occur simultaneously.

\section{Solid Molecular Hydrogen at Low-temperature}

Diamond anvil experiments have made many important discoveries concerning the phase diagram of solid molecular hydrogen at high pressure (Mao and Hemley, 1994). Figure 3 shows the experimental phase diagram of solid $\mathrm{H}_{2}$ and $\mathrm{D}_{2}$ at high pressure and low temperatures. The three low-temperature phases firmly established experimentally are labeled as phases I, II and III in figure 3. Note that in the literature, the latter two phases are also known as the Broken Symmetry Phase (BSP) and the hydrogen- $A$ (H- $A$ ) phase, respectively.

Starting at low densities, anisotropic intermolecular interactions between hydrogen molecules are weak. Because of this, the angular momentum of an individual molecule $(J)$ remains a good quantum number. Furthermore, since there is a large separation of the rotational energy levels, only the $J=0$ (para- or $p$ - $\mathrm{H}_{2}$ ) or $J=1$ 
(ortho- or $o-\mathrm{H}_{2}$ ) states are thermally populated at low temperatures. At relatively low pressures and temperatures, para- $\mathrm{H}_{2}$ molecules freely rotate about their center of mass (i.e., they have a spherically symmetric wavefunction, and the molecular bond angles are completely disordered, even at $T=0 \mathrm{~K}$ ), while ortho- $\mathrm{H}_{2}$ can align at very low temperature. Further, at low pressures, the interconversion between ortho- and para- is very slow and the phase diagram is sensitive to their concentration, because ortho- and para- molecules interact differently. It has been found that $p-\mathrm{H}_{2}$ at low pressure crystallizes into the close-packed hcp structure (Hazen et al., 1987; Keesom et al., 1930), referred to as Phase I, whereas molecules in $\mathrm{o}-\mathrm{H}_{2}$ orient along the body diagonals of an fcc structure with Pa3 symmetry (Clouter and Gush, 1965; Mills and Schuch, 1965; Mills et al., 1973; Yarnell et al., 1974). An extensive review of the low pressure theory and experiment for solid molecular hydrogen is given in Silvera, 1980.

As the pressure is increased, at temperatures lower than $140 \mathrm{~K}$, the rotational symmetry of Phase I is broken, and hydrogen transforms into Phase II (hence the designation as the BSP). The structure of Phase II is determined by zero point rotational energy of the molecules. Hence, the phase I-II boundary is sensitive to isotope as well as temperature; at very low temperatures, the transition to the Phase II occurs at 27.8 GPa in $o-\mathrm{D}_{2}$ (Silvera and Wijngaarden, 1981), 70 GPa in HD (Moshary et al., 1993), and $110 \mathrm{GPa}$ (Lorenzana et al., 1990).

As the pressure is further increased to $\sim 150 \mathrm{GPa}$, molecular hydrogen undergoes another transition (Hemley and Mao, 1988; Lorenzana et al., 1989) to Phase III. The thermodynamic stability range of Phase III has recently been experimentally demonstrated to extend to pressures over $300 \mathrm{GPa}$ and temperatures up $300 \mathrm{~K}$ (Zha et al., 2012). The transition into Phase III is more robust; the transition pressure is relatively insensitive to the isotope, hinting that Phase III is determined by the BornOppenheimer energy of interacting static molecules.

Experimental measurements have only been able to provide limited information about the molecular orientations in phases II and III. The small sample sizes and low x-ray scattering efficiency (proportional to the atomic number squared) pose challenges to structural identifications using x-rays. However, recent measurements up to $183 \mathrm{GPa}$ indicate that the center of each molecule remains close to the Phase I hcp lattice sites (Akahama et al., 2010) in both phases II and III. The basic hcp hexagonal symmetry was also seen in neutron scattering measurements of Phase II $\left(\mathrm{D}_{2}\right)$ (Goncharenko and Loubeyre, 2005), also showing evidence for partial orientational ordering.

The other important experimental constraint comes from Raman spectroscopy. At the Phase II-III transition, a jump in the intermolecular vibron (Hemley and Mao, 1988; Lorenzana et al., 1989) and a large increase in absorbance of the IR-active vibron are observed. Also, the number of low-frequency Raman-active modes and possible second Raman vibron indicate that in Phase III, the primitive cell should contain at least four molecules (Goncharov et al., 1998). Further, the fact that the IR and Raman vibrons and phonons have different frequencies implies a center of symmetry. The presence of Raman-active phonons means that any center of inversion is between molecules. Three IR modes and one Raman mode are observed in Phase II (Cui et al., 1995).

There have been many attempts to identify the structures of phases II and III, and using various types of simulation methods. But as is discussed below, a consensus has not yet been reached.

At relatively low pressures (thus pertinent to both o$\mathrm{H}_{2}$ in Phase I and Phase II), an appropriate simplified model of solid molecular hydrogen has found extensive use (Freiman et al., 2003); this model consists of quantum rotors fixed on a lattice and interacting as quadrupoles (Felsteiner, 1965; Nakamura, 1955):

$$
H=\sum_{i=1}^{N} B \mathbf{L}_{i}^{2}+\sum_{i<j} V\left(\Omega_{i}, \Omega_{i} ; \mathbf{R}_{i j}\right) .
$$

Here $B$ is the rotational constant (the presence of which highlights expected differences in isotopes), $\mathbf{L}_{i}$ is the angular momentum operator of rotor $i, \Omega_{i}$ is its orientation, $\mathbf{R}_{i j}$ is the vector between rotors $i$ and $j$ and $V$ the interaction energy between two quadrupoles. Note that an array of ordered (classical) electric quadrupoles (EQ) has a lower energy in an fcc lattice than in hcp, explaining the observed structure and ordering observed in $o-\mathrm{H}_{2}$. While this assumes classical rotors, it was later supported with an analysis of the quantum mechanical rigid-rotor model (Raich and James, 1966). Since the observed lattice is hcp, presumably effects not described in the above Hamiltonian favor the hcp versus fcc structure.

There have been several simulations of extensions of the quantum rotor model, e.g. the PIMC simulations by (Runge et al., 1992) to Phase II. These calculations used a more accurate pairwise intermolecular potential derived from LDA calculations. They found that $o-\mathrm{D}_{2}$ would indeed order first into a Pa3 structure, but then transform to an ordered hcp structure, while $p-\mathrm{H}_{2}$ would order directly into an ordered hcp structure. Unfortunately, while qualitatively consistent with what one would expect (e.g., the isotope effect of the transition as well as finite-temperature effects), a group theoretical analysis showed that both structures are quantitatively incompatible with the observed number of IR (3) and Raman (1) modes (Cui et al., 1995).

Later calculations expanded on the initial quantum rotor calculations. PIMC calculations by Surh et al., 1997 considered both fcc and hcp lattices of rotors, finding a structure with $P 6_{3} / m$ symmetry, a hexagonal structure where some molecules are in-plane and some perpendicular. Again, the group theoretical analysis by Cui et al., 1995 also rules out $P 6_{3} / m$, due to an insufficient number of IR vibrons. Although, the PIMC calculations by Cui et al., 1997 suggested a Pa3-type ordering, similar to that 
in $p-\mathrm{H}_{2}$ and $o-\mathrm{D}_{2}$ at low-pressure and temperature (i.e., molecules along the body diagonals), but remaining in a hcp lattice. Interestingly, this latter prediction has recently been suggested to be qualitatively (but not necessarily quantitatively) consistent with neutron diffraction of $\mathrm{D}_{2}$ in a $P-3$ structure (Goncharenko and Loubeyre, 2005). It is clear that while the quantum-rotor model appears to provide insight into phases I and II, quantitative predictions for Phase II remain lacking, which is presumably attributable to approximations in the underlying potential between rotors.

Other approaches to predict molecular orientations are based on fully $a b$ initio calculations, including DFT or QMC. Typically, these have been based on evaluating $\mathrm{BO}$ energies for static lattices, which as will be discussed below, neglects important contributions to the energetics from proton ZPM and thermal effects. The applicability to Phase II is therefore questionable. An early QMC simulation (Ceperley and Alder, 1987a), predating the experimental discovery of phases II and III, and did, in fact, include ZPM), led to the prediction of a phase transition at roughly the observed pressure (110 GPa). However, the trial wavefunctions employed and computer resources available for those calculations were very limited compared with those currently available. Nonetheless, that calculation importantly demonstrated that the energies involved in localizing the molecular orientation were such that the transition should occur at roughly the observed pressure.

A number of candidate structures have been proposed for Phase II (and III) such as Cmc2 $2_{1}$ (Kitamura et al., 2000), $P 2_{1} / c$ (Johnson and Ashcroft, 2000; Zhang et al., 2007), and Pca2 $2_{1}$ (Kohanoff et al., 1997; Nagao et al., 1999; Städele and Martin, 2000). The lattices and molecular orderings are shown in Fig. 4. Besides differing molecular orientations, they structures are all similar, consisting of orthorhombic primitive cells with lattice sites close to those of an hcp structure.

Among the structures shown in Fig. 4, $\mathrm{Pca} 2_{1}$ has perhaps been the most thoroughly studied, and is considered a likely candidate for Phase II. Pca2 $2_{1}$ was first suggested as the ground-state of a classical quadrupolar system (Kitaigorodskii and Mirskaya, 1965), and then later proposed as the ground-state of $\mathrm{H}_{2}$ based on DFT calculations (Nagara and Nakamura, 1992). DFT calculations employing an exact-exchange functional further showed that indeed $P c a 2_{1}$ is the most stable static structure (Städele and Martin, 2000).

However, the correctness of $P c a 2_{1}$ is called into question by the Raman experiments; a single mode is actually observed, while there should be four if the structure is indeed $P c a 2_{1}$. An explanation might found in either a relatively small signal-to-noise ratio or cancelations between various vibrational modes (Cui et al., 1995; Kohanoff et al., 1997).

Recently, methods to determine the crystal structure have been proposed (Woodley and Catlow, 2008), and some have been applied to hydrogen. These methods
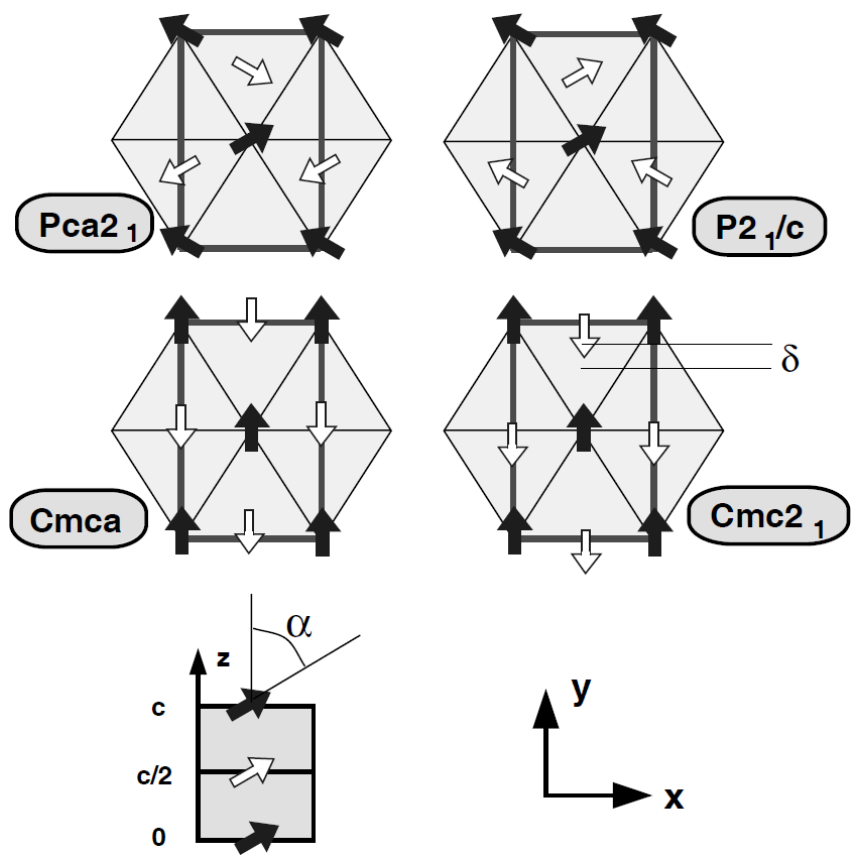

FIG. 4 Possible molecular orientations (indicated via arrows) for dense hydrogen (phases II and III), assuming 4 molecules per unit-cell (Städele and Martin, 2000). The solid (empty) arrows represent molecules at $c(c / 2)$ lattice points (out of the plane). Molecules are tilted with respect to the $c$ axis at an angle $\alpha \sim 55^{\circ}$, and $\delta$ indicates the distance of each molecular center of mass from the hcp lattice site.

attempt to find the most stable crystal structure over a much broader class of lattices. In the $a b$ initio random structure searching (AIRSS) method (Pickard and Needs, 2006), for example, one relaxes a number of randomly chosen unit cells by minimizing the BO-DFT energy. After a large number of attempts, one assumes that the correct structure has been found. Such AIRSS calculations have been applied to $\mathrm{H}_{2}$ by Pickard and Needs, 2007, once again finding the $P c a 2_{1}$ and $P 6_{3} / \mathrm{m}$ structure suggested earlier. However, as mentioned above and which we will further elaborate on below, these searches do not directly take into account ZPM, and thus even if the assumed density functional were sufficiently accurate, would not necessarily correspond to the physical structures. To date, the searches are based on determining molecular orderings with classical protons, and adding an approximate ZPE at a second step. Dynamical lattice calculations using DFT linear-response theory, (Zhang et al., 2006) demonstrated that $P c a 2_{1}$ remains stable if ZPE is taken into account in comparison to other examined structures in the pressure range $110 \leq P \leq 150$ GPa. Furthermore, even though this approach lacks rotational motion, an examination of the rotational energies of a set of candidate structures, including the $P 6_{3} / \mathrm{m}$ structure, suggests that rotational motion in $P c a 2_{1}$ is the least energetic (Moraldi, 2009). Although, recent PIMD calculations suggests the possibility that the structure of Phase II is isotope dependent (Geneste et al., 2012), with 


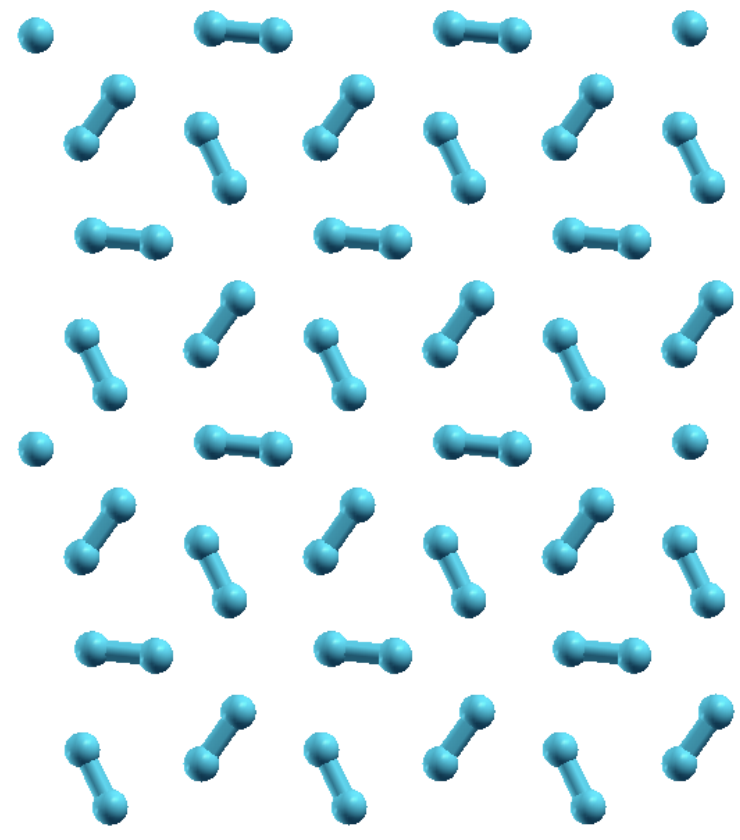

FIG. 5 The most likely candidate for Phase III of hydrogen, the $C 2 / c$ structure predicted by Pickard and Needs, 2007. As indicated in the image and discussed in the text, this structure consists of rings of three molecules, responsible for the strong optical activity.

the description of hydrogen in terms of a single classical structure not possible.

Early on, Barbee III et al., 1989 used DFT to predict Phase III structures, finding molecular bonds aligned with the $c$-axis of hcp lattice. However, later DFT calculations found more stable structures with molecules oriented in the $(a, b)$ plane (Kaxiras and Broughton, 1992; Kaxiras et al., 1991). QMC calculations by (Natoli et al., 1995) agreed with this assessment, finding the orientation angle to be roughly $60^{\circ}$ as shown in Fig. 4 and consistent with the experimental data. Note that molecular orientation along $c$ will not exhibit IR activity. By considering larger unit cells Nagara and Nakamura, 1992 found the hexagonal $\mathrm{Pca} 2_{1}$ structure, shown in Fig. 4. However, this structure is inconsistent with phase III (Cui et al., 1995). Finally, a structure search using 12 molecules close to distorted hcp lattice (Pickard and Needs, 2007), found the most stable structure to be $C 2 / c$ with sites consistent with the limited diffraction data (Akahama et al., 2010)) (see Fig. 5). This agrees qualitatively well with the spectroscopic data for phase III, including intense IR activity, because the molecules in this structure are no longer symmetric, and have a dipole moment.

As can be seen, the use of computational methods to predict the structures of phases II and III of hydrogen has an unexpected complexity. While qualitative insight has been provided, the results have not been quantitative: $P c a 2_{1}$ and $C 2 / c$ are plausible candidates for phases II and III, respectively, but open questions remain.

Although methods to find systematically the lowest en- ergy structures have made impressive advances recently (Woodley and Catlow, 2008), albeit at a greatly increased computational cost, challenges remain. One problem is to estimate the effect of proton ZPM accurately; we will return to this issue in Section IV.A.6. By estimating the ZPE using frozen-phonon calculations, Surh et al., 1993 suggested that $c$-oriented molecules in an hcp lattice were actually more stable than $P c a 2_{1}$, contrary to the initial static-lattice predictions. Of course, more exact estimates of ZPM could be treated via a PIMD, PIMC, or QMC (Ceperley and Alder, 1987a) approach, at an increased computational cost. Secondly, structure searching relies on DFT, the accuracy of which depends on the assumed functional. Many calculations employ local or semi-local (GGA) exchange DFs which do not adequately describe vdW at low pressure, while at higher pressure severely underestimate the band gap (Städele and Martin, 2000). As discussed in Section II.H, recent advancements have been made to improve DFs so that one may hope for much more reliable predictions in the very near future. It is clear that more work needs to be done both theoretically and experimentally to map out the molecular phases.

\section{Solid Molecular Hydrogen at High-temperature}

Experiments and calculations suggest that at least two additional phases of hydrogen may exist at elevated temperatures.

Goncharov et al., 1995 noticed a change in the slope of the I-III phase-line of $\mathrm{D}_{2}$ along with a subtle discontinuity of the intramolecular vibron as a function of pressure. In addition, the observed discontinuity in the vibron frequency across the I-III transition line become vanishingly small above $254 \mathrm{~K}$. It was suggested that there is another phase, denoted by I', located at higher temperture than phase I $(T \geq 170 K)$ and isostructural with phase III in with a critical (or tricritical) point where the vibron discontinuity vanishes (see figure 5 of ref. (Goncharov et al., 1995)). A further support in favor of phase I'was provided by a PIMC calculations based on the quantum rotor model (see Section IV.A.1) by Surh et al., 1997. However, the accuracy of the used effective intermolecular potentials are unknown. Further experimental support to Phase I'was provided by Baer et al., 2007, 2009 using coherent anti-Stokes Raman spectroscopy on deuterium samples. Comparing the pressure dependence of the Raman shift of the $D_{2}$ vibron along two isotherms, at $77 \mathrm{~K}$ and at $300 \mathrm{~K}$, they observed a change of slope in the difference around $140 \mathrm{GPa}$ which they ascribed to the signature of the phase transition from Phase I to Phase I'along the $300 \mathrm{~K}$ isotherm, in agreement with Surh et al., 1997 and in qualitative agreement with the early picture of Goncharov et al., 1995. However, to reconcile the two different experiments, the I-I'phase line would need to have a negative slope (see fig. 3) and should exhibit a strong temperature dependence not observed in the early 


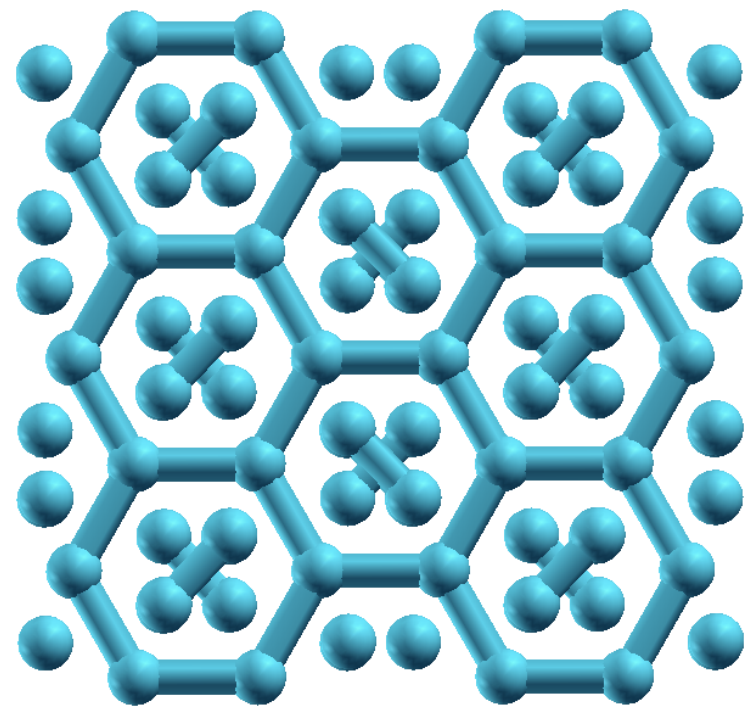

FIG. 6 (Color online) Theoretical and experimental prediction for phase IV (Howie et al., 2012; Pickard et al., 2012). The structure consists of graphene-like layers.

experiments (Goncharov et al., 1995). Recently, Goncharov et al., 2011 performed a more refined study and concluded that the new data and analysis do not support the existence of phase $\mathrm{I}^{\prime}$. As can be seen, uncertainties still remain surrounding the existence and the details of phase I'and further systematic investigations are necessary.

Recently Raman and visible transmission spectroscopy measurements at room temperature $(T \sim 300 K)$ (Eremets and Troyan, 2011; Howie et al., 2012) suggest yet a further phase-transition, an entropically-driven (and reversible) one to a new Phase IV ${ }^{6}$. For example, after compressing to phase III, Howie et al., 2012 observed three spectroscopic signals near $220 \mathrm{GPa}$ indicative of a phase transition: (i) the appearance of the second fundamental vibrational mode, (ii) a dramatic softening and broadening of the first fundamental vibrational mode, and (iii) the appearance of new low-frequency phonon excitations.

The Raman spectra suggest that two distinct local environments exist in Phase IV (Howie et al., 2012); and by comparing this data to the previously-predicted ground-state structures of solid hydrogen (Pickard and Needs, 2007), Howie et al., 2012 suggest that Phase IV could be a mixture of graphene-like layers and unbound $\mathrm{H}_{2}$ molecules, such as shown in Fig. 6. Note that the graphene-like layers are shown with equal distances between protons in Fig. 6 (corresponding to the Ibam structure of Pickard and Needs, 2007.) However, experiments suggest that equal bond distances only occur at higher

\footnotetext{
${ }^{6}$ A transition to a high pressure-high temperature phase was already reported (Mao and Hemley, 1989) but not characterized
}

pressure; at lower pressures, $\mathrm{H}_{2}$ dimers undergo pairing fluctuations (Howie et al., 2012), possibly resulting in the lower-symmetry Pbcn structure (Pickard and Needs, 2007).

Immediately following the experimental evidence for Phase IV (Eremets and Troyan, 2011; Howie et al., 2012), AIRSS was re-applied to the problem of high-pressure molecular hydrogen (Pickard et al., 2012), using larger unit cells than previously. A number of consistent mixed phases, similar to Ibam (Fig. 6) were found, but with space groups $P c$. Particularly interesting about these is that by estimating the ZPE and its entropic contribution to the free energy within the quasi-harmonic approximation, they become thermally stable relative to the presumed structure for phase III, C2/c (Pickard and Needs, 2007), consistent with experiment (Howie et al., 2012). One must keep in mind that the errors involved in the quasi-harmonic approximation and the used density functional can be larger than the differences in energies of the various proposed structures.

Around the same time of these measurements and calculations, however, Zha et al., 2012 performed IR and optical absorption measurements using DACs at similar thermodynamic points that were studied by Eremets and Troyan, 2011; Howie et al., 2012. Their measurement did not reveal strong evidence for, or even suggest, a transition to a new phase, however, such a transition could not be completely ruled out.

\section{Additional Solid Molecular Phases}

DAC measurements indicate that the thermodynamic stability range of Phase III is quite large; the strong IR absorption of the vibron (Goncharov et al., 1998) was demonstrated to persist up to at least $320 \mathrm{GPa}$ at relatively low temperatures (Goncharov et al., 2001; Loubeyre et al., 2002). More recent measurements (Zha et al., 2012) have extended this range even further, to $360 \mathrm{GPa}$ at low temperatures as well as up to $300 \mathrm{~K}$ at somewhat lower pressures. Computational predictions support the large stability range of the molecular phase, but at the same time suggest that additional solid phases may exist at elevated pressures.

The recent AIRSS study by Pickard and Needs, 2007 (see Section IV.A.1) predicted not only a plethora of structures for phases II and III, but also suggested two further phase transitions. Above $285 \mathrm{GPa}$, these searches (Pickard and Needs, 2007) and a more recent analysis (Pickard et al., 2012) suggest that Phase III will transform to a structure with $C m c a$ symmetry and 12 atoms

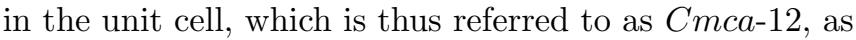
shown in Fig. 7. $\mathrm{Cmca-12}$ is rather similar to the (presumed) structure of Phase III ( $C 2 / c$, Fig. 5); for example, consisting of three-molecule rings, except the layering in Cmca-12 has the form ...ABA..., the molecules lie flat within each layer, and the distortion of the molecular centers from hcp packing is larger. 

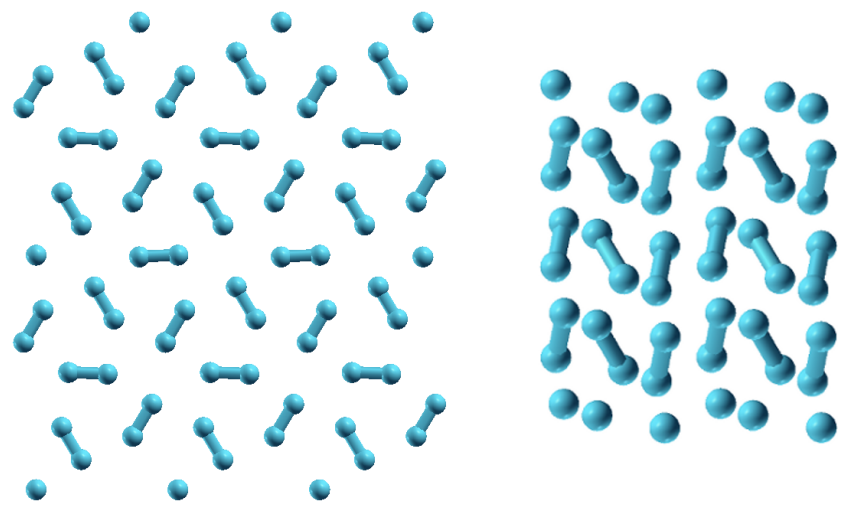

FIG. 7 (Color online) A single layer of the $C m c a-12$ structure (Pickard and Needs, 2007) at $300 \mathrm{GPa}$ (left) and the $C 2 / c(2)$ structure (Liu et al., 2012) at $500 \mathrm{GPa}$ (right). Due to the higher compression, $C 2 / c(2)$ has been enlarged relative to Cmca-12. The other predicted high-pressure molecular phase, $\mathrm{Cmca}$, occurring at pressures intermediate between $\mathrm{Cmca-12}$ and $C 2 / c(2)$, is shown in Fig. 4.

Considering ZPM in the quasi-harmonic approximation, the transition from Phase III is predicted to be mainly pressure driven (i.e., temperature-independent), occurring at either 240GPa (Pickard and Needs, 2007) or at $\sim 285 \mathrm{GPa}$ (Pickard et al., 2012), as indicated in the phase diagram in Fig. 3. One might wonder why the transition to $\mathrm{Cmca-12}$ has not been detected in the DAC experiments (Goncharov et al., 2001; Loubeyre et al., 2002; Zha et al., 2012). However, Pickard et al., 2012 suggest that the drop in the IR vibron frequency observed between $\sim 292-330 \mathrm{GPa}$ (Zha et al., 2012) is consistent with a phase transition to $\mathrm{Cmca-12}$. Of course, it is also plausible that there are inaccuracies in the calculations that affect these predictions, such as the DF employed in the DFT calculations or the perturbative treatment of proton ZPM, both of which are expected to become increasingly important with increasing pressure. Further calculations and experiments will be necessary to assess the accuracy of these predictions.

The AIRSS study by Pickard and Needs, 2007 also predicted a further phase transition at $385 \mathrm{GPa}$, from $\mathrm{Cmca}$ 12 to $C m c a$ originally proposed by (Edwards et al., 1996); that structure is shown in Fig. 4.

Very recently, an alternative to AIRSS, the particleswarm optimization (PSO) method for structure prediction (Wang et al., 2010), was applied to dense hydrogen (Liu et al., 2012) and found a another stable $\mathrm{H}_{2}$ phase beyond $\mathrm{Cmca}$ in the pressure range $470-590 \mathrm{GPa}$. Interestingly, this structure possesses two different nearestneighbor proton separations (which can roughly be called intramolecular bonds), in a space group $C 2 / c(2)$ [we have added the designation (2) to distinguish it from the $C 2 / c$ structure predicted for Phase III], also shown in Fig. 7. Furthermore, these bond distances are larger than the free-space intramolecular separation of the Cmca structure $(\sim 0.88 \AA$, compared to $0.74 \AA$ or $0.78 \AA$, respectively), suggesting a sequence of structures that occur as the system changes from molecular to atomic hydrogen.

Labet et al., 2012a,b,c,d recently considered the process of molecular dissociation in depth, by focusing on the relationship between and variations of intra- and intermolecular bond lengths with pressure: more precisely the shortest $\left(r_{\mathrm{H}-\mathrm{H}}(P)\right)$ and second-shortest $\left(R_{\mathrm{H}_{2}-\mathrm{H}_{2}}\right)$ proton distances. To quantify their results, an equalization function $\xi(P)$ at pressure $P$ is defined as,

$$
\xi(P)=1-\frac{R_{\mathrm{H}_{2}-\mathrm{H}_{2}}(P)-r_{\mathrm{H}-\mathrm{H}}(P)}{R_{\mathrm{H}_{2}-\mathrm{H}_{2}}\left(P_{1 \mathrm{~atm}}\right)-r_{\mathrm{H}-\mathrm{H}}\left(P_{1 \mathrm{~atm}}\right)} .
$$

$\xi$ is an order parameter, evolving from $\xi=0$ (free-space molecules) to 1 (an atomic state where all proton distances are equal) during dissociation. Focusing on the structures predicted in the AIRSS study by Pickard and Needs, 2007, Labet et al., 2012a,b,c,d found a discontinuous shift at the transition from $\mathrm{Cmca}$ to the atomic phase, leading them to propose an intermediate phase exists allowing continuous dissociation. While the proposed static lattices have a higher energy than $\mathrm{Cmca}$ (Edwards et al., 1996; Pickard and Needs, 2007) and $C 2 / c(2)$ (Liu et al., 2012)], those continuous structures could be stabilized by proton ZPM, not included in their calculations. Nonetheless, the results offer insight into molecular dissociation, and suggest a continuous path that may occur as the molecular lattice is compressed.

\section{Melting curve of the molecular crystal}

Significant progress has been made both experimentally and computationally in determining the melting curve of hydrogen, as recently reviewed in Silvera and Deemyad, 2009. At ambient pressure, $\mathrm{H}_{2}$ and $\mathrm{D}_{2}$ crystallize into an hcp structure at temperatures of 14 and $19 \mathrm{~K}$, respectively (Silvera, 1980). Before the development of DAC techniques, measurements of the melting line of hydrogen were limited to pressures below $\sim 2$ GPa (Liebenberg et al., 1978). DAC experiments (Diatschenko and Chu, 1981; Diatschenko et al., 1985) extended the melting line to pressures above $7 \mathrm{GPa}$ and fit to a modified Simon equation. With improvements in DAC technology and heating techniques, Datchi et al., 2000 and Gregoryanz et al., 2003 were able, by monitoring the shift in the Raman-active vibron, to measure the melting line up to 15 and $44 \mathrm{GPa}$, respectively, as reported in Fig. 8. The measurements by Gregoryanz et al., 2003 found a decrease in the slope of the melting line with respect to pressure, and suggested a maximum in the curve. This maximum was, in fact, suggested earlier by Datchi et al., 2000 , on the basis of an extrapolation of the Kechin melting curve, which has a maximum in the melting line near $128 \mathrm{GPa}$ and $1100 \mathrm{~K}$.

Using constant pressure CPMD simulations with classical protons, Scandolo, 2003 predicted that the the melting line at higher pressures will have a negative slope, as a consequence of a liquid-liquid phase-transition (see Section IV.B.2). Following that prediction, two-phase (solid 


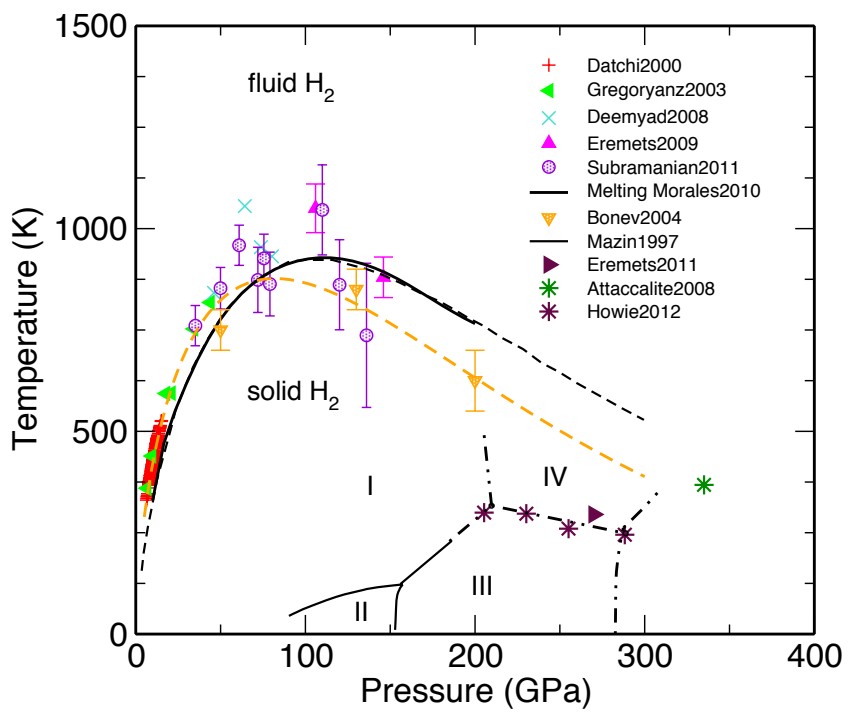

FIG. 8 Reentrant melting line of the Phase I. Experimental data: red crosses (Datchi et al., 2000), green left-triangles (Gregoryanz et al., 2003), turquoise X's (Deemyad and Silvera, 2008), violet up-triangles (Eremets and Trojan, 2009) and purple dashed circles (Subramanian et al., 2011). Theoretical predictions: BOMD (orange triangles (Bonev et al., 2004a)), free-energy calculations (solid black line (Morales et al., 2010b)). The orange dashed-curve is a fit of the Datchi, Gregoryanz and Bonev data to a Kechin equation, while the black dashed curve is a fit to the Morales data using the same functional form. A stable fluid point predicted by MD using QMC forces (green star (Attaccalite and Sorella, 2008)). Shown below the melting curve are the experimental solid phases (identical to Fig. 3 including recently reported metallic transition (Eremets and Troyan, 2011).

and liquid) CPMD simulations were performed to trace the melting line to higher pressures (Bonev et al., 2004a). A maximum in the melting line below $1000 \mathrm{~K}$ was found, as well as a negative slope that extrapolates to $0 \mathrm{~K}$ near $400 \mathrm{GPa}$. Of course, such extrapolations do not take into account phase changes in the liquid or solid phases at higher pressures. These calculations were recently corroborated by Morales et al., 2010b up to a pressure of $200 \mathrm{GPa}$ : the melting line of hydrogen was calculated by comparing the (DFT) Gibbs free-energy of the liquid and solid molecular phases (Phase I, hcp rotationally disordered). Recent measurements using laser heating of hydrogen in a DAC (Deemyad and Silvera, 2008) have observed a maximum and subsequent decrease of the melting temperature with increasing pressure. These measurements are consistent with those more recently reported by Eremets and Trojan, 2009; Subramanian et al., 2011. The various measurements and theoretical predictions are shown in Fig. 8.

The comparison of the experimental data to the DFT predictions of melting for pressures between 10 and 140 GPa is a gratifying confirmation of their accuracy. However, we note that at pressures when metallization is oc- curring on the melting line (estimated to be at about $250 \mathrm{GPa}$ and $500-600 \mathrm{~K}$ ), one expects local or semi-local forms of the assumed DFT functional to bias the results and predictions using these functionals to be much less accurate. Also, we note that the effect of the quantum motion of the protons on the melting line tends to cancel out only if the crystal and liquid phase are both molecular or both atomic. Otherwise, ZPM of the protons needs to be taken into account in determining the melting temperature. Despite this expected inaccuracy, the Kechin equation (Kechin, 2004a,b) $T_{m}(K)=$ $14.025\left(1+P_{m} / a\right)^{b} \exp (-P / c)$ has been used to extrapolate the low pressure data to higher pressures. In Fig. 8 , two such extrapolations are reported. The first one, reported using an orange dashed line, considers experimental points from Datchi et al., 2000 and Gregoryanz et al., 2003 and FPMD points from Bonev et al., 2004a, suggesting $a=0.030355, b=0.59991, c=137$, while the second one considers only simulation results from freeenergy calculations (Morales et al., 2010b), suggesting $a=0.1129, b=0.7155, c=149$. These extrapolations, suggest that at higher pressure, the molecular crystal phase might vanish in favor of a low-temperature liquid phase with very unusual properties (see Section IV.C.2). However, extrapolations based on the low pressure crystal structures are highly susceptible to error.

\section{The Metallization of solid Molecular Hydrogen}

Wigner and Huntington, 1935 predicted that hydrogen would undergo an insulator-to-metal (IM) transition at sufficiently high pressure. For the general Hamiltonian in Eq. (1), one can easily show that the potential energy scales as $r_{s}^{-1}$ while the kinetic energy as $r_{s}^{-2}$. Hence, as the density and pressure increase $\left(r_{s} \rightarrow 0\right)$, the kinetic energy will dominate. Since the free particle wavefunction minimizes the kinetic energy, this implies that any electronic system will go to an uncorrelated wavefunction: a simple metal. To determine precisely how and when hydrogen at low temperature becomes a metal has been a major question, and, has been termed the "holy grail" of high-pressure physics.

We note that at non-zero temperature, there is always some thermal excitation of carriers, and thus some conductivity, so a precise definition of the IM transition is only possible at zero temperature. In a later section, we will discuss the shock experimental measurements of the conductivity of (Nellis et al., 1999; Weir et al., 1996), where the IM transition was observed at high temperature $(\sim 2600 \mathrm{~K})$ near $140 \mathrm{GPa}$ in the liquid hydrogen phase. See also recent reviews (Maksimov and Shilov, 1999; Robitaille, 2011).

The early assumption was that the IM transition would be associated with molecular dissociation to the atomic state (Abrikosov, 1954; Wigner and Huntington, 1935). However, Hartree-Fock calculations using the exact exchange operator (Ramaker et al., 1975) as well as band- 
structure calculations (Friedli and Ashcroft, 1977) suggested that metallization may instead occur in the molecular phase; as change in density causes the molecular bands to shift around, there can be a band gap closure(Mazin and Cohen, 1995), either direct or indirect. This can occur when the bandwidths of the $1 \sigma_{g}$ and $1 \sigma_{u}$ bands of the molecular orbitals become larger than their splitting, and thus overlap. Initial predictions suggested molecular metallization near $150 \mathrm{GPa}$, and so it was thought that the phase II $\rightarrow$ III transition could be the onset of metallization (Hanfland et al., 1993). But this is not the case, and finding the transition remains an open problem; both how it occurs and at what pressure.

Metallization of solid molecular hydrogen has been reported in several experiments (Maksimov and Shilov, 1999), most recently in DAC experiments near $300 \mathrm{~K}$ and $\sim 265 \mathrm{GPa}$ (Eremets and Troyan, 2011). However, opinions concerning the results have been mixed (Jephcoat, 2011; Nellis et al., 2012). Further, other DAC experiments near the same thermodynamic conditions by Zha et al., 2012 report a semi-metallic state, rather than a metal. At lower temperatures, however, the experimental results agree that insulating molecular hydrogen exists up to the highest pressure currently achievable, $320 \mathrm{GPa}$ (Goncharov et al., 2001; Loubeyre et al., 2002). Extrapolations of optical measurements of the band-gap provide an estimate that closure should occur between pressures of 325 - $450 \mathrm{GPa}$ (Goncharov et al., 2001; Loubeyre et al., 2002). This of course assumes that there are no further crystal structure changes above $320 \mathrm{GPa}$, and that gap closes smoothly as a function of pressure.

If it is difficult for the DAC to achieve metallization, predicting the (IM) transition also remains challenging. The first problem arises from the ignorance of the high pressure crystal structure, as discussed earlier. Since each crystal structure has a much different arrangement of bands, without the knowledge of the crystal structures, one cannot say much about the metallization(Barbee III et al., 1989). Transition pressure predictions have thus varied greatly, ranging, for example, from $200-450$ GPa. Since the most recent predictions have converged on the Cmca phase Fig. 4) as the most likely candidate for high-pressure molecular hydrogen (Johnson and Ashcroft, 2000; McMahon and Ceperley, 2011a; Pickard and Needs, 2007), its electronic structure repeatedly examined.

In addition to this challenges, there are also difficulties in calculating the effects of the electron correlation and zero point motion of the protons. Most calculations have used the local or semi-local DFT functional which are known to underestimate band gaps (Perdew and Levy, 1983; Sham and Schlüter, 1983) implying that the IM transition density will be underestimated (Oliva and Ashcroft, 1981a,b). See Fig. 9 where this effect has been calculated in molecular hydrogen. The error in the band gap is related to lack of the derivative discontinuity of the exchange-correlation energy, as well as the self-interaction error and can be reduced by using

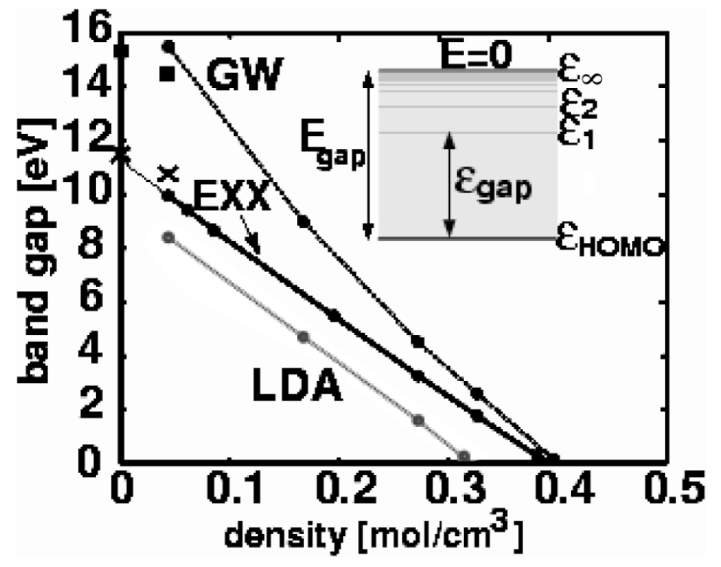

FIG. 9 Electronic band gaps for molecular hydrogen in a hcp lattice with molecules oriented along the $c$ axis (Städele and Martin, 2000). No zero point motion was included.

the much more computationally expensive quasiparticle methods (Chacham and Louie, 1991), methods that include exact-exchange (Städele and Martin, 2000), and calculations based on the many-body $G W$ method (Johnson and Ashcroft, 2000). These calculations have shown that the band gaps in hydrogen are indeed underestimated by $\sim 1-2 \mathrm{eV}$ in molecular hydrogen, as can be seen in Fig. 9, for molecular hydrogen in an hcp lattice. Note that exact-exchange calculations slightly underestimate the gap (Jones and Gunnarsson, 1989). With such increased gaps, the IM transition is predicted to occur near pressures of at least $400 \mathrm{GPa}\left(\sim 0.4 \mathrm{~mol} / \mathrm{cm}^{3}\right)$, in agreement with experimental extrapolations (Goncharov et al., 2001; Loubeyre et al., 2002).

Another issue which has received much less attention is the effect of zero point motion of the protons. If one can assume that the molecular phase has the protons confined near lattice sites, then we can treat the ZPM as an effect of electron-phonon coupling. It is known that this can affect both the magnitude of the band gap, and its dependence on density and temperature (Allen and Cardona, 1981a,b). It is reasonable to expect that the influence of this should be especially important in molecular hydrogen, where the electronphonon coupling is particularly strong (Cudazzo et al., 2008, 2010a,b) (see also Section IV.C.1). However, treating this self-consistently within the framework of DFT calculations, i.e., non-perturbatively, including its effect on band gaps and energetics is currently not straightforward. One can, however, envision the possibility of such calculations using fully ab initio QMC calculations (Ceperley and Alder, 1987b; Natoli et al., 1995), which also would not suffer the band gap problems discussed above. 


\section{Atomic crystal phases}

In a landmark publication, Wigner and Huntington, 1935 suggested that molecular hydrogen would dissociate to an atomic state at high pressure, and further that any Bravais lattice of such a state would be metallic. Their predictions were that the atomic phase would become energetically favorable at $\sim 25 \mathrm{GPa}$. Of course, this prediction was long before the availability of numerical methods or experimental techniques to create such pressures. Ever since this prediction, the pressure required to form this state has been pushed ever upwards, but the transition to the atomic lattice is yet to be observed. The most recent predictions (Liu et al., 2012; McMahon and Ceperley, 2011a; Pickard and Needs, 2007) suggest that at least $\sim 500 \mathrm{GPa}$ will be required.

The most fundamental property, for which the predictions of other properties rely on, is the crystal structure. Naively, one might expect hydrogen to assume a relatively simple crystal structure because at high pressures, as we discussed in the previous section, the system will become a free-electron metal (Ashcroft and Mermin, 1976). At sufficiently high density, the lattice of protons will interact with the bare, unscreened Coulombpotential; and, as is well-known, the structure minimizing the Coulomb potential is the bcc lattice, which was thus the primary structure considered in Wigner and Huntington, 1935.

However, it has turned out that the alkali metals, such as lithium and sodium, that are free-electron like at ambient conditions and should become moreso under pressure, exhibit rather exotic and complex pressure-induced structural transitions (Rousseau et al., 2011). The simple scaling argument fails because even a small amount of electronic screening of the proton-proton interaction is enough to destabilize the bcc lattice. It is plausible that atomic hydrogen will instead undergo an analogous sequence of transitions as a function of temperature and pressure.

Some of the early work proposed anisotropic structures, such as layered ones (Brovman et al., 1972a; Kagan et al., 1977) reminiscent of the graphite-type structure [also considered by Wigner and Huntington, 1935], as well as filamentary structures (Ebina and Miyagi, 1989; Nagara, 1989). Anisotropic structures were also considered by Barbee III et al., 1989 and Barbee III and Cohen, 1991, the latter study drawing a structural analogy with the $9 R$ ground-state structure of ambientpressure lithium (Overhauser, 1984). Other studies, however, predicted isotropic structures (Ceperley and Alder, 1987a; Nagao et al., 1997; Natoli et al., 1993; Straus and Ashcroft, 1977).

A central challenge to modeling hydrogen and these predictions is the accurate treatment of proton ZPM, as discussed in Section II.E.2. While the simple harmonic approximation, Eq. (40), continues to be used (Liu et al., 2012; McMahon and Ceperley, 2011a; Pickard and Needs, 2007), Straus and Ashcroft, 1977 demonstrated that the
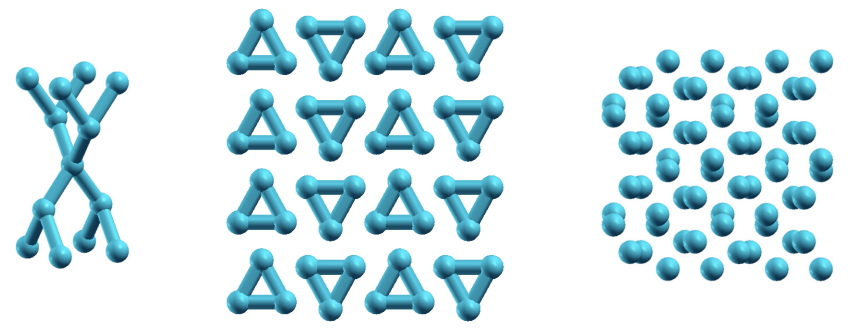

FIG. 10 (Color online) Predicted ground-state structures of atomic metallic hydrogen: (left) I4 $/$ amd at $500 \mathrm{GPa}$ (McMahon and Ceperley, 2011a; Nagao et al., 1997; Pickard and Needs, 2007); (middle) Cmcm at 2.5 TPa (Liu et al., 2012); (right) $I-43 d$ at $3.5 \mathrm{TPa}$ (Liu et al., 2012). Bonds are drawn between nearest neighbors.

simple harmonic approximation can fail, using a family of face-centered tetragonal structures (similar, in fact, to those considered again in later studies (Nagao et al., 1997)). Only by including anharmonicity, for example, were some structures found to be energetically stabilized, in this case the isotropic fcc structure. Later calculations Natoli et al., 1993 confirmed these findings via $T=0 \mathrm{~K}$ QMC calculations, demonstrating that ZPM indeed favors isotropic structures. Since the ZPE of the protons can be larger than the difference in energy between various candidate structures, it is important to realize that structure searching, even including an approximate ZPE should be approached with caution.

Following more recent advancements in structure prediction methods (Woodley and Catlow, 2008), as discussed in prior sections, McMahon and Ceperley, 2011a considered atomic hydrogen, predicting a large number of previously unidentified structures. Their work suggested that after molecular dissociation, hydrogen should adopt a structure similar to Phase IV of cesium, a bodycentered tetragonal structure with space group $I 4_{1} /$ amd and a $c / a$ ratio greater than unity, consistent with earlier predictions (Nagao et al., 1997; Pickard and Needs, 2007). This structure was found to be stable at least up to $\sim 1$ $\mathrm{TPa}$, including a harmonic estimation of ZPE. More recently, Liu et al., 2012 applied the PSO method also to the atomic phase of hydrogen, predicting two novel structures at ultrahigh-pressures (i.e., beyond the stability range of $\left.I 4_{1} / a m d\right)$. Above $2.1 \mathrm{TPa}$, atomic hydrogen adopts a structure with space group $\mathrm{Cmcm}$, consisting of planar $\mathrm{H}_{3}$ clusters, as shown in Fig. 10. The $\mathrm{Cmcm}$ structures is in fact analogous to the enthalpically-favored static-lattice structure $R 3 \mathrm{~m}$ predicted by McMahon and Ceperley, 2011a at such pressures, but with a more efficient packing. Liu et al., 2012 also suggested that above $3.5 \mathrm{TPa}$, a structure with space-group $I-43 d$, also shown in Fig. 10, would become stable with the inclusion of ZPE (albeit again treated within the harmonic approximation).

At very high density and relatively low temperature, it is expected that atomic hydrogen should adopt a closepacked lattice, such as fcc or hcp (Kohanoff and Hansen, 
1996). In this regime, one can "integrate out" the electrons and use a screened pair potential between the protons, as discussed in Section II.G.1. The limit of validity of this Screened Coulomb Plasma (SCP) model $\left(r_{s} \leq 0.6, P \geq 20 \mathrm{TPa}\right)$ was established by comparing against CPMD predictions (Kohanoff and Hansen, 1996), expected to be accurate at high density, and further verified against more accurate CEIMC calculations (Liberatore et al., 2011b; Pierleoni et al., 2008). Free energy calculations of the SCP at 24TPa give an estimated melting temperature of $1670 \mathrm{~K}$. As mentioned in Section IV.C.2), if the melting temperature is low enough, a very interesting quantum fluid could be stable. However, more investigations are needed to determine the stable crystal structures at lower pressures and their melting temperatures.

\section{B. The Normal Fluid Phases}

While an understanding of the low-temperature region of the phase diagram of hydrogen is certainly important for fundamental reasons, the major fraction of hydrogen in the universe is in the fluid phase at higher temperatures. What is of particular importance under these conditions is an accurate EOS that can be used for planetary models, and to validate the computational assumptions and experimental procedures of the dynamical shock experiments. Theoretical and computational methods have progressed to the point where sufficient accuracy can now be obtained to resolve some of their past difficulties. In this subsection, we discuss first-principles calculations applied to the fluid phase of hydrogen, with particular focus on predicting the principal Hugoniot of deuterium and hydrogen and the liquid-liquid phase transition (LLT).

\section{The Equation of State and Principal Hugoniot of Hydrogen}

The EOS of hydrogen in the fluid phase is particularly relevant for planetary and ICF physics (see Fig. 1). As discussed in Section III, experimental information for the liquid phase of hydrogen comes mainly from dynamic compression experiments, with the exception of the low temperature liquid near the melting line which can be studied using static compression techniques. Particularly useful properties such as the electrical conductivity or reflectance, can be measured during the experiment and are difficult to compute with simulations. However, dynamic compression methods have large uncertainties in determining the pressure, and, particularly, the temperature of the sample after the shock, and, therefore, obtaining precise thermodynamic data. Also, since the experiments are difficult and rather expensive, the reached set of temperature-density points is rather sparse. Because of this, first-principles simulation methods are very useful to complement the experimental data.
Early shock wave experiments on hydrogen and deuterium samples were done with a gas-gun compression method (van Thiel and Alder, 1966; van Thiel M. et al., 1973) up to pressure of $20 \mathrm{GPa}$ in the first shock and 90 $\mathrm{GPa}$ in the reflected shock, and by laser-driven compression methods (van Kessel and Sigel, 1974) up to pressures of $\sim 200 \mathrm{GPa}$ in a single shock experiment. Soon after, the first measurements of electrical conductivity in isentropically compressed hydrogen in a magnetic-flux compression device appeared (Hawke et al., 1978) which reported a measured drop of electrical resistivity below 1.0 $\Omega \mathrm{cm}$ at a density of $1.06 \mathrm{~g} / \mathrm{cm}^{3}$ and an estimated pressure of $200 \mathrm{GPa}$. Continuous progress in the experimental techniques provided better data for the first (singleshock) Hugoniot up to $20 \mathrm{GPa}$ and the second (reflectedshock) Hugoniot (Nellis et al., 1983a,b) up to 76 GPa, see figure 11. However, the temperature was not measured; the estimated temperature was based on a phenomenological chemical model of fluid hydrogen(Kerley, 1972; Ross et al., 1983). By measuring the electrical conductivity in the deuterium and hydrogen shocked samples, (Nellis et al., 1992) it was inferred that, below $20 \mathrm{GPa}$, shocked fluid deuterium behaves as a hot semiconductor with a band gap of $\sim 12 \mathrm{eV}$.

After the introduction of FPMD methods (Car and Parrinello, 1985) for studying s-p bond materials, application to high pressure hydrogen with an orbital-free method was made by Zerah et al., 1992 and by a ground state DFT-LDA Car-Parrinello calculation(Hohl et al., 1993). This was subsequently followed by several studies including thermal effects on the electrons using the Mermin functional (Alavi et al., 1994; Collins et al., 1995; Kohanoff and Hansen, 1995, 1996; Kwon et al., 1995, 1994a,b) and an inclusion of excited states within the TDDFT-LDA framework by Theilhaber, 1992. These calculations were plagued by systematic errors due to limitations of the LDA energy functional and of small system sizes, in particular in the metallic phase. Almost in parallel, the first RPIMC calculations of the EOS and the liquid-liquid phase transition (see next section) in high pressure hydrogen appeared (Magro et al., 1996; Pierleoni et al., 1994, 1996). While FPMD studies concentrated on a density range above the density of the principal Hugoniot, RPIMC results, limited to temperature above $5000 \mathrm{~K}$, also probed the region of phase space of the principal Hugoniot. RPIMC found a nonmonotonous behavior of the pressure vs density along the Hugoniot which was interpreted as the signature of a first-order phase transition between a molecular fluid and an atomic fluid. Unfortunately, in this first implementation of RPIMC, systematic effects due to system size and imaginary time step errors limited the accuracy of the results. Wave-Packet MD was also applied to high pressure hydrogen (Klakow et al., 1994a,b). In this method, electrons are represented by single-electron Gaussian wave packets; Ehrenfest dynamics is used to simulate the electronic dynamics and to extract the forces acting on the protons. The approach is, however, limited to the semi- 


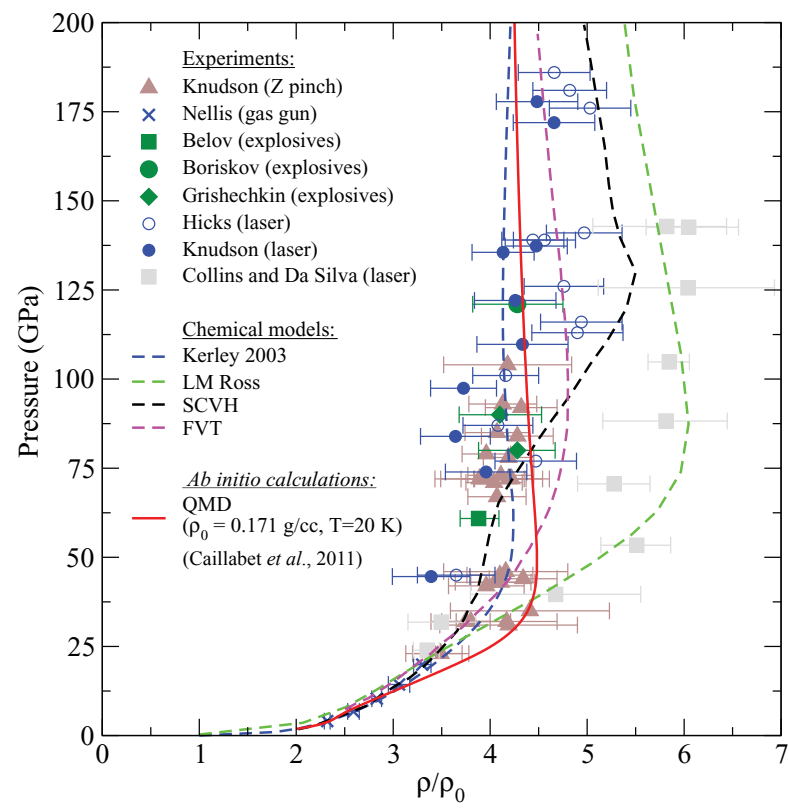

FIG. 11 (Color online) Comparison between the measured and calculated principal Hugoniot for deuterium. The experimental data are plotted as symbols with error bars: Z machine (triangles)(Knudson et al., 2004), gas gun (crosses)(Nellis et al., 1983b)), explosives (green squares) (Belov et al., 2002), (large circles)(Boriskov et al., 2003), (diamonds)(Grishechkin et al., 2004a), and laser (open circles) (Hicks et al., 2009), (filled small circles) (Knudson and Desjarlais, 2009), and (gray squares)(Collins et al., 1998a; DaSilva et al., 1997). The red line is the Hugoniot from (Caillabet et al., 2011) Predictions of various chemical models are reported as dashed lines: Kerley (Kerley, 2003b), Ross(Ross, 1998), Saumon-Chabriervan Horn(Saumon et al., 1995) and FVT (Juranek and Redmer, 2000). From Caillabet et al., 2011.

classical regime since Fermi statistics is only accounted for approximately and its applicability to the phenomena of molecular dissociation and metallization is only qualitative.

Nellis et al., 1996 and Holmes et al., 1995 directly measured the temperature along the Hugoiniot by fitting the radiance of the light emitted from the shocked sample to a grey-body Plank spectrum; see fig. 13. They found temperatures substantially lower than predicted by the employed chemical model, with an increasing discrepancy with increasing pressure. This was interpreted as an indication of a substantial molecular dissociation above 20 GPa.

Principal Hugoniot pressures up to $\sim 340 \mathrm{GPa}$ were later achieved with a pulsed laser-produced shock compression (Celliers et al., 2000; Collins et al., 1998a; DaSilva et al., 1997); see fig. 11. Those experiments reported a much higher compressibility, and therefore, higher shock density, up to a 6 -fold compression, $\left(\rho / \rho_{0} \sim\right.$ $6)$; higher than expected by the standard EOS ((Kerley, 1972)) which does not include molecular dissociation. Such a high compression would have a dramatic effect on the efficiency of Inertial Confined Fusion. The unexpected compression was not incompatible with some existing theoretical predictions and models which allowed for molecular dissociation. Note that at sufficiently high pressure, the compression on the principal Hugoniot must attain the ideal gas value of 4 (Nellis, 2006a). Along the Hugoniot the system was observed, by optical reflectivity measurements (Celliers et al., 2000), to undergo a continuous insulator to metal transition in the region between $17 \mathrm{GPa}$ to $50 \mathrm{GPa}$ as inferred by a continuous increase in the reflectance signal (from 10\% to 50\%) and a saturation at higher pressures. Temperature was determined by pyrometric measurements (Collins et al., 2001a) and was found to increase from $0.47 \mathrm{eV}$ to $4.4 \mathrm{eV}$ (5000-50000 $\mathrm{K}$ ) in the pressure range from $31 \mathrm{GPa}$ to $230 \mathrm{GPa}$ (see fig. 13). This temperature is smaller than the Fermi temperature of the metallic fluid $(\sim 16 \mathrm{eV})$, the samples are a degenerate metal. Improvement in compression (up to $\sim 12$-fold) has been reported in reflected-shock experiments where the second shock occurs at $\sim 100 \mathrm{GPa}$ along the primary Hugoniot line(Mostovych et al., 2001, 2000). Pressures up to $600 \mathrm{GPa}$ were reported. However, the temperature was not measured during these experiments. The results are again in much closer agreement with phenomenological models including molecular dissociation.

These exciting experimental results stimulated new first-principle simulation studies. Attempts to compute the electrical conductivity to have a direct comparison with experiments appeared (Pfaffenzeller and Hohl, 1997). This study found a more pronounced metallicity and atomic character than reported in the experiments probably attributable to the use of LDA. Further tight-binding MD found a principal Hugoniot in substantial agreement with the gas-gun data below $20 \mathrm{GPa}$ but could not reproduce the laser-driven data at higher pressure(Lenosky et al., 1997a,b,c, 1999). Two independent ab-initio MD studies using DFT with Gradient corrected exchange-correlation functional (GGA) were performed(Collins et al., 2001b; Galli et al., 2000; Lenosky et al., 2000). A further study which exploited the LSDA in the AIMD was also performed(Bagnier et al., 2000). The results of the three studies were all in agreement and support a maximum compression of $\sim 4.4$ in the region of $50 \mathrm{GPa}$, with a limiting compression for higher pressure around 4 in substantial disagreement with the laser-driven Hugoniot. On the other hand the computed optical reflectivity was in good agreement with the measurements performed during the laser-driven runs up to $70 \mathrm{GPa}($ Collins et al., 2001b). The system was found to smoothly dissociate along the Hugoniot reaching a value of the electrical conductivity of $4000(\Omega \mathrm{cm})^{-1}$ at maximum compression. Improved RPIMC simulation was also performed with smaller time step and fintesize errors and a better nodal restriction taking into account bound states (Militzer and Ceperley, 2000). As can be seen in figure 12, this calculation provided an almost vertical Hugoniot with maximum compression of 


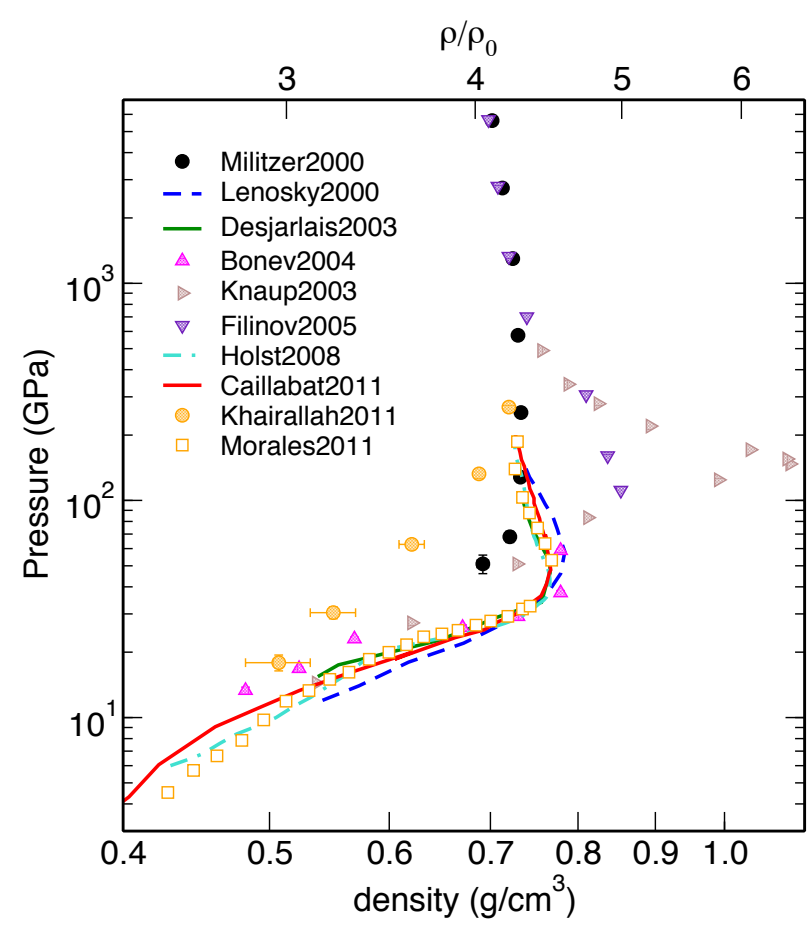

FIG. 12 (Color Online) Comparison among various theoretical methods of computations of the principal Hugoniot for deuterium. RPIMC (black circles)(Militzer and Ceperley, 2000), (orange circles)(Khairallah et al., 2011) and direct PIMC (cyan down-triangles) (Filinov et al., 2005). FPMD ground state electrons (red dot-dashed line) (Lenosky et al., 2000), (blue squares) (Bonev et al., 2004b) and thermal electrons (continuous violet) (Desjarlais, 2003), (dot-dashed brown)(Holst et al., 2008), (continuous purple) (Caillabet et al., 2011) and (green up-triangles)(Morales et al., 2012). WPMD (gray right-triangle) (Knaup et al., 2003)

$4.3 \pm 0.1$ also in disagreement with laser-driven shock experiments. In a subsequent study combining RPIMC $\left(\mathrm{T}>10^{5} \mathrm{~K}\right)$ and first-principles $\mathrm{MD}\left(\mathrm{T}<10^{5} \mathrm{~K}\right)$ simulation techniques (Militzer et al., 2001), the secondary Hugoniot was investigated and results compared with the experimental data of Mostovych et al.(Mostovych et al., 2001, 2000). Even in this case it was found that RPIMC and AIMD were in essential agreement but both at variance with the secondary Hugoniot from laser-driven experiments.

Magnetic implosions at the $\mathrm{Z}$ pinch were then used to create shock waves in much larger samples of liquid deuterium(Knudson et al., 2001). Pressures of $\sim 70 \mathrm{GPa}$ were achieved with a 4.0-4.5-fold compression, in contrast to the 6 -fold compression observed in the laserdriven experiments, see fig. 11. This data is in much closer agreement with the first-principle predictions of RPIMC and FPMD. Finally, a new technique based on spherically converging shock waves generated by explosives(Belov et al., 2002) (data reported in figure 11) also produce lower compressibilities.
A subsequent refinement of the detection technique of the magnetic pressure apparatus (Knudson et al., 2003) and further experiments (Knudson et al., 2004) measured the principal Hugoniot up to 100 GPa confirming a maximum compression of $\sim 4.3$ (see fig. 11) and provided data along the second shock Hugoniot up to 400 $\mathrm{GPa}$ and final density of deuterium of $1.34 \mathrm{~g} / \mathrm{cm}^{3}$. In those experiments, temperature was not measured but later experiments using reflectivity and emissivity, to infer the temperature(Bailey et al., 2008) are shown in figure 13 , and found to be in good agreement with previous data. Also, second-shock data up to $900 \mathrm{GPa}$ has been measured with laser-driven shocks at OMEGA laser in Rochester (Boehly et al., 2004). These data are consistent with a stiff EOS with 4.3 to 4 .4-fold maximum compression along the principal Hugoniot. A further experimental confirmation of a stiff EOS along the principal Hugoniot came from converging explosive driven shock waves(Boriskov et al., 2005; Grishechkin et al., 2004a) (see fig. 11).

Recently, new laser-driven experiments using impedance matching to an aluminum standard have been performed (Hicks et al., 2009). At variance with previous experiments, they found 4.2-fold compression for pressures near $100 \mathrm{GPa}$, in agreement with other experiments based on the impedance matching method. However, just above $100 \mathrm{GPa}$ and up to the highest reached pressure (220 GPa), a sudden jump to higher compression $(\sim 5)$ was observed, but soon after (Knudson and Desjarlais, 2009), it was shown that this behavior was caused by an erroneous calibration of the quartz impedance matching standard used to infer the principal Hugoniot above $100 \mathrm{GPa}$. At present, after 20 years of efforts, a consensus is emerging on the principal Hugoniot of deuterium obtained by several different experimental methods and teams.

While most previous experimental work has been on deuterium samples, a recent experiment using laserdriven shock waves(Sano et al., 2011a) measured shocked hydrogen in the pressure range between $25 \mathrm{GPa}$ and 55 $\mathrm{GPa}$. The lower pressure data are in agreement with previous experiments (Dick and Kerley, 1980; Nellis et al., 1983a) and at higher pressures they show a compression of $\sim 5$, which suggest that hydrogen is more compressible than deuterium. However, the measured temperature is higher in hydrogen by a factor of $\sim 1.3$.

In parallel with advances in experimental methods and results, first-principle methods also have been improved and extended. At the turn of the century, two FPMD studies investigated some of the systematic errors: the first used Born-Oppenheimer dynamics with thermal electrons (Desjarlais, 2003) while the second used CarParrinello dynamics with ground state electrons(Bonev et al., 2004b). As seen in figure 12 consistent results were obtained. The computed Hugoniot was slightly stiffer than in a previous work (Lenosky et al., 2000) but still had a maximum compression of about 4.5 at $\sim 40 \mathrm{GPa}$, and in agreement with the gas gun Z-pinch 
experiment up to $20 \mathrm{GPa}$ (Knudson et al., 2001). Above this pressure, the new theoretical Hugoniot was slightly stiffer than the experimental data. Also, the predicted temperature along the Hugoniot for $P \leq 20 G P a$ was found to be in very good agreement with the experimental gas-gun data (see fig. 13). In contrast, previous firstprinciple studies predicted temperatures smaller by as much as 30\% (Lenosky et al., 2000). Several later BOMD simulations using DFT-GGA confirmed these results: Vorberger et al., 2007b was limited to lower temperatures, used ground state electrons, and investigated the hydrogen-helium mixture (see Section V), Holst et al., 2008 used thermal electrons and computed optical properties such as reflectivity and conductivity (see below).

We should also mention a number of theoretical investigations which predicted a soft principal Hugoniot. The first study was performed by Wave-Packet Molecular Dynamics (Knaup et al., 2003) and reported a principal Hugoniot with a maximum compression of $\sim 6$ in agreement with the Nova laser data (see fig. 12). The accuracy of the WPMD is however limited to the semiclassical regime and should not be applied at these temperatures. Also, the predicted temperature was considerably lower than in the experiments and in the first-principle methods. The second study employed the so-called Direct Path Integral Monte Carlo (DPIMC) method (Filinov et al., 2005) and reported a maximum compression of $\sim 5$ at $P=111 G P a$, providing a softer EOS with respect to the RPIMC predictions of Militzer and Ceperley, 2000, see fig. 12. Also in this method, the temperature along the Hugoniot was substantially lower than in experiments and in other first-principle methods (see fig. 13). Finally, we mention a recent investigation of the principal Hugoniot by the antinodal-slice RPIMC method (Khairallah et al., 2011) (see section II.C). They obtain good agreement with the previous RPIMC Hugoniot at high pressure $(P \geq 200 G P a)$ and a stiffer Hugoniot at lower pressure, but still compatible with the experimental data within their large uncertainty (see fig. 12). However, these results do not agree well with the low pressure gas-gun data. Also, as seen in fig. 13, the predicted temperature along the Hugoniot is somewhat higher than the experimental measurements, and the predictions from RPIMC and FPMD.

As can be seen in Fig. 11, the uncertainty on the experimental data is large, which makes it difficult to discriminate between different calculations. On the other hand, the most recent FPMD calculations of the principal Hugoniot give similar results and are in agreement with the experimental data, reinforcing the consensus on the experimental Hugoniot.

Validation of the FPMD predictions can be obtained either by comparing with experimental data or by comparing to results from a more fundamental method such as CEIMC. The two methods solve the electronic problem in very different ways and with different approximations: CEIMC is based on the variational principle (with respect to the nodes of the trial wave function), can be

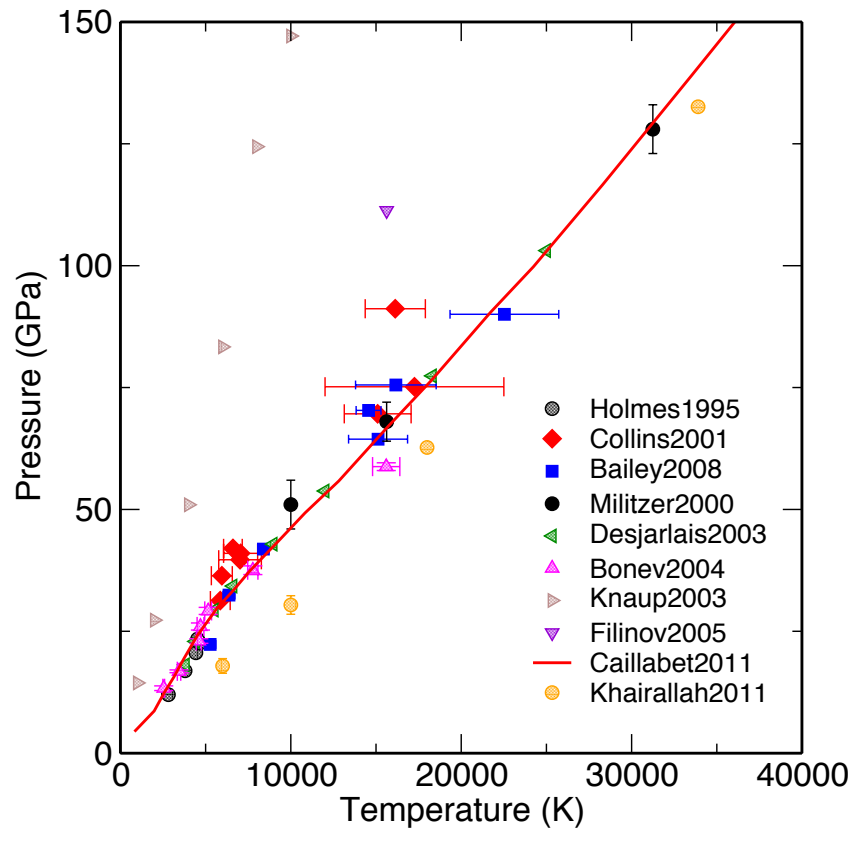

FIG. 13 (Color Online) Pressure vs temperature along the Hugoniot. Comparison among experimental data and various theoretical predictions. Experiments: gas-gun (red circles (Holmes et al., 1995)), Nova-laser (cyan diamonds (Collins et al., 2001a)), Z-pinch (blues squares (Bailey et al., 2008)). Theory: RPIMC (black circles (Militzer and Ceperley, 2000), orange circles (Khairallah et al., 2011)), BOMD (green lefttriangle (Desjarlais, 2003), magenta up-triangle (Bonev et al., 2004b), red line (Caillabet et al., 2011)), direct PIMC (purple down-triangle (Filinov et al., 2005)) and WPMD (brown right-triangle (Knaup et al., 2003)).

systematically improved. CEIMC is a relatively recent method, rather more demanding than FPMD, therefore its applications have been limited so far. Moreover, earlier implementations were plagued by insufficient accuracy of the trial wave function and gave results in disagreement with CPMD (LDA) predictions at higher density around the melting transition of the atomic metallic crystal $\left(0.8 \leq r_{s} \leq 1.2,500 \mathrm{~K} \leq T \leq 10000 \mathrm{~K}\right)$ (Pierleoni et al., 2004). Recent methodological progress, in particular the implementation of LDA-Kohn-Sham orbitals in the Slater determinant of the trial wave function, improves considerably the accuracy and the flexibility of the method (Pierleoni et al., 2008) and provides results for the hydrogen EOS which are in good agreement with the predictions of FPMD (Morales et al., 2010a; Pierleoni et al., 2008). Morales et al., 2010a compared the EOS from both methods in the region $2000 \mathrm{~K} \leq T \leq 10000 \mathrm{~K}$ and $0.724 \mathrm{~g} / \mathrm{cm}^{3} \leq \rho \leq 2.329 \mathrm{~g} / \mathrm{cm}^{3}\left(1.55 \geq r_{s} \geq 1.05\right)$, corresponding to $100 \mathrm{GPa} \leq P \leq 2000 \mathrm{GPa}$, a small region slightly to the right of the first Hugoniot (see Fig. 1) and relevant in modeling the Jovian planets. The pressure difference between the two methods was at most $5 \%$ at the lowest density (approaching the molecular dissociation regime) and decreases with increasing density. 
The difference in the internal energy $(\sim 0.8 \%)$ appears to be uniform with density. Also, predictions for the local structure of the proton fluid are in very good agreement. As we will see in the next section, this agreement is not observed at the metal-insulator transition underlying again the non-trivial character of this finding. Our present understanding is that DFT-based FPMD is quite accurate in comparison to CEIMC, both at high pressure and at low pressure in regions away from the metal-insulator transition. In the region where the system become metallic the local and semi-local functionals have a well known problem of underestimating the band gap and FPMD provides wrong predictions: see below.

Most of the theoretical studies discussed so far concentrated on computing the first and second Hugoniots. Very recently, first-principle calculations have been performed over a much wider region in thermodynamic space (Caillabet et al., 2011; Hu et al., 2011; Morales et al., 2012) to build a thermodynamically consistent EOS for conditions appropriate to planetary models and ICF applications. Morales et al., 2012 used both FPMD and CEIMC to study the EOS in the range $10 G P a \leq P \leq$ $1000 G P a, 3000 K \leq T \leq 35000 K$. It was found that the Kerley2003 EOS works well after a pressure dependent, but temperature independent, correction is applied. Hu et al., 2011 employed RPIMC to map the EOS in the range $0.002 \mathrm{~g} / \mathrm{cm}^{3} \leq \rho \leq 1596 \mathrm{~g} / \mathrm{cm}^{3}, 10^{4} \mathrm{~K} \leq T \leq 10^{8} \mathrm{~K}$. Again the RPIMC-based EOS is in good agreement with the Kerley2003 table. We note that an earlier PIMC calculation of the low density phase diagram of hydrogen (Militzer and Ceperley, 2001) reported a substantial agreement between PIMC data for the EOS and the Saumon-Chabrier chemical model predictions. On the other hand, in the higher pressure range investigated in Ref. Morales et al., 2010a (100GPa $\leq p \leq 2000 G P a)$ the modified SCVH EOS (Saumon et al., 1995) was found to deviate from the CEIMC data by as much as $\sim 25 \%$ at the edge of the dissociation region, the region in which chemical models are essentially based on interpolations. Because planetary models are sensitive to details in this regime and at lower pressures during dissociation, a deviation from SCVH will produce a much larger change - e.g., it is found using a less compressible EOS that Jupiter has a core mass of 14-18 earth masses, much larger than SCVH value of 0-7 earth masses (Guillot, 2005; Militzer et al., 2008). However, consensus on the interior model for Jupiter and the EOS is yet to be reached.

\section{Liquid-Liquid Phase Transition}

Fluid hydrogen at low pressure is $\mathrm{H}_{2}$; molecular dissociation will occur both with increasing temperature or with pressure. At moderate pressures $\left(\sim 10^{2}-10^{4}\right.$ bar), molecular dissociation happens when the temperature is approximately equal to the molecular bonding energy $(\sim 4.5 \mathrm{eV})$. The dissociation temperature depends mildly on pressure since internal degrees of freedom (either molecular or atomic) are largely independent of pressure as shown in fig. 2 . This prediction is based on chemical models which are expected to be accurate at low density (Hu et al., 2011; Saumon et al., 1995). On the other hand, with increasing pressure at moderate temperature $(T \sim 1000 K)$, the physics becomes more complex because the separation between internal degrees of freedom and many particle interactions disappears; the internal states of the molecules are strongly influenced by molecule-molecule interactions, and, for high enough pressure, molecules dissociate and the system becomes metallic.

Whether dissociation and metallization occur at the same time and whether the processes are continuous are open questions with recent experimental and theoretical findings. The occurrence of metallization induced by pressure was discussed by Wigner and Huntington, 1935 for atomic hydrogen at $\mathrm{T}=0 \mathrm{~K}$, by Landau and Zeldovich, 1943 for liquid mercury as a first order phase transition, and by Norman and Starostin, 1970 for a dense atomic plasma. In high pressure fluid hydrogen, molecular dissociation and ionization was discussed by Ebeling et al. (Ebeling and Richert, 1985; Ebeling and W., 1985) and later by other authors (Beule et al., 1999; Edwards et al., 2010; Fortov et al., 2003a; Kitamura and Ichimaru, 1998; Marley and Hubbard, 1988; Redmer and Holst, 2010; Saumon and Chabrier, 1989, 1991, 1992) at several levels of sophistication in the framework of chemical models. These models exhibit a clear first-order liquid-liquid transition (LLT) that persists for temperatures well above 10,000 K. However, approaches with separate free energy functionals in different regions of phase space have great difficulty in having a continuous crossover from one behavior to another. The presence of a first-order phase transition in the chemical models is now recognized to be an artifact of the method(Chabrier et al., 2007). Recent EOS tables now smooth the pressure in the transition region to ensure positive compressibility (Saumon, 2007).

Nellis et al., 1996 and Weir et al., 1996 (see also (Nellis et al., 1999)), using reverberating shocks to achieve quasiisoentropic compression up to $180 \mathrm{GPa}$, found that the resistivity decreased by almost 4 orders of magnitude in a continuous manner(Nellis et al., 1998) between $93 \mathrm{GPa}$ to $140 \mathrm{GPa}$, and then saturated to roughly $500 \mu \Omega \mathrm{cm}$ between $140 \mathrm{GPa}$ and $180 \mathrm{GPa}$, a typical resistivity value for liquid metals. They estimated the temperature from a model at $140 \mathrm{GPa}$ to be $\sim 3000 \mathrm{~K}$ as shown in fig. 14 . They estimated the molecular dissociation to be $\sim 5 \%$ : the system is still largely molecular.

More recently, Fortov et al., 2007 using reverberating shocks, ramp compressed hydrogen with high explosives, and found a discontinuous behavior at the metalinsulator transition. Using highly resolved flash x-ray diagnostics, they were able to measure the compressibility of the liquid and found a $20 \%$ increase in density in the regime where the conductivity increases by $\sim 5$ orders of 


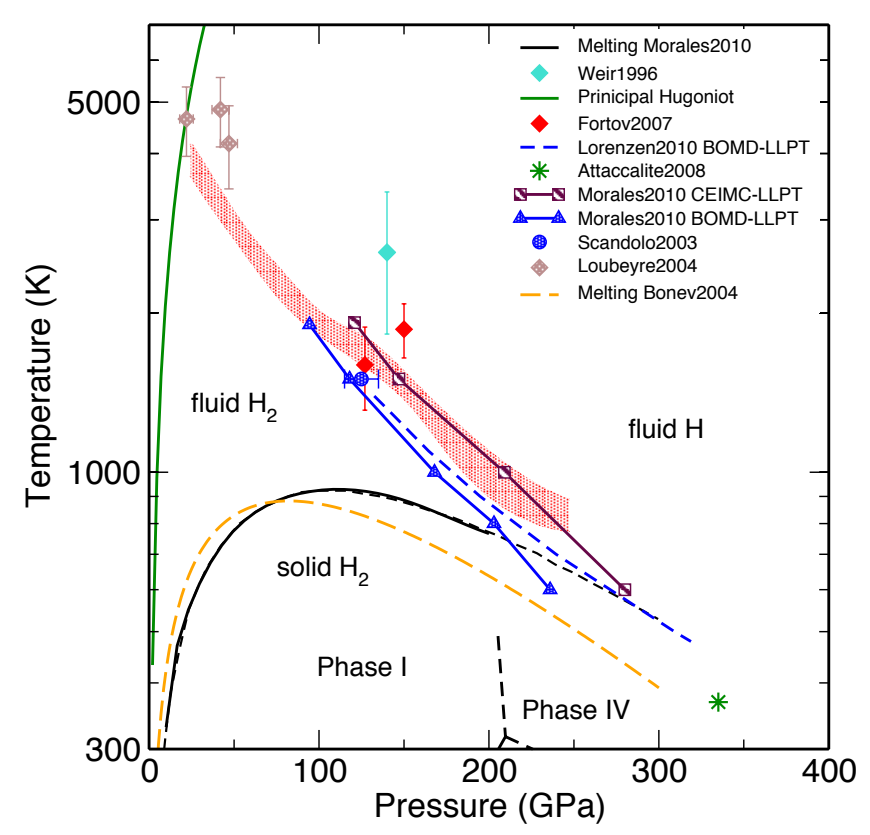

FIG. 14 Liquid-liquid transition line and pressure dissociation region. Experimental data for metallic hydrogen: (cyan diamond (Nellis et al., 1996; Weir, 1998), red diamonds (Fortov et al., 2007), brown diamonds (Loubeyre et al., 2004)). Theoretical predictions for the LLPT: (blue triangle BOMD) (Morales et al., 2010b) and (brown squares CEIMC (Liberatore et al., 2011a; Morales et al., 2010b)) The blue and brown lines are a guide to the eye through BOMD and CEIMC simulation data respectively. (blue circle (Scandolo, 2003), dashed blue line (Lorenzen et al., 2010)). Molecular dissociation region according to BOMD: red-shaded area (Tamblyn and Bonev, 2010a). Primary Hugoniot: thick green line. QMC prediction of stable fluid state: green star (Attaccalite and Sorella, 2008). Melting curve of the molecular solid (phase-I) is represented by two possible curves as discussed in section IV.A.4: solid (dashed) black line (Morales et al., 2010b), orange dashed line (Bonev et al., 2004a). The expected boundary between phase I and phase IV is also reported (Howie et al., 2012).

magnitude. The temperature was not measured directly but inferred from a chemical model EOS to be in the range of $3000 \mathrm{~K}$ to $8000 \mathrm{~K}$. The two data points around the density discontinuity (using the EOS from a recent FPMD study(Tamblyn and Bonev, 2010a)) are shown in figure 14. However, the measurements from this experiment were sparse in density and a rapid, yet continuous, change in conductivity with increasing pressure could not be ruled out from the data.

The first $a b$ initio evidence of a first order phase transition came from a Restricted PIMC calculations (with free particle trial nodes) showing an abrupt pressure change along isotherms(Magro et al., 1996) with a critical point at $T_{c} \simeq 11000 K, P_{c} \simeq 48 G P a$ in qualitative agreement with prediction by chemical models. However, the RPIMC method has major problems with convergence at temperatures below $10000 \mathrm{~K}$ and that calculation was plagued by finite imaginary time step errors.

The next step towards the present understanding of liquid-liquid phase transition in hydrogen came from a CPMD (using DFT-GGA energy functional) and classical protons(Scandolo, 2003) in the constant pressure ensemble. A first order phase transition was signalled by large density fluctuations at $T=1500 K$ and $P=$ $125 \mathrm{GPa}$ with a $6 \%$ change of specific volume. At the transition, a rapid molecular dissociation was observed and the system became metallic with the gap at the Fermi level closing, both molecular dissociation and metallization of hydrogen occurring together with a first order phase transition. This result is not in contradiction with the dynamical compression experiments of Weir et al. (Nellis et al., 1996; Weir et al., 1996) but requires the existence of a critical temperature between $1500 \mathrm{~K}$ and 3000 K. Note that the temperature in the shock experiments was estimated by an indirect method. Also, the transition pressure of first-principle DFT-GGA simulations is somehow lower than measured in the experiments; see fig. 14. Evidence of a first order liquid-liquid phase transition was also found by Bonev et al., 2004a,b using the CPMD method with the GGA functional in the NVT ensemble at pressures of $200 \mathrm{GPa}$ and temperatures between 900 $\mathrm{K}$ and $1000 \mathrm{~K}$. But a smooth dissociation process under pressure was found in a subsequent BOMD investigations (Holst et al., 2008; Vorberger et al., 2007a,b). A region with $(\partial P / \partial T)_{\rho}<0$ along isochores inside the dissociation region $(P \simeq 200 G P a, 1000 K \leq T \leq 4000 K)$ was reported without a density discontinuity but a continuous crossover from an insulating to state.

Similarly, a CEIMC simulation (Delaney et al., 2006) reported no evidence for a first order phase transition at $\mathrm{T}=1500 \mathrm{~K}$ and $\mathrm{T}=2000 \mathrm{~K}$ in the pressure range from $135 \mathrm{GPa}$ to $290 \mathrm{GPa}$. In this work, the trial wave function had a Slater determinant using the orbitals from a band-structure solution with an effective electron-proton potential. Employing VMC energies along the isotherm at $\mathrm{T}=2000 \mathrm{~K}$, a jump in the molecular fraction was observed when increasing density around $220 \mathrm{GPa}$ with an hysteresis when releasing the density. However, when using RQMC energies at the same conditions, the molecular fraction presented a much smoother behavior with density, suggesting a continuous dissociation process.

Finally, a convincing demonstration of the first order liquid-liquid phase transition was found by Liberatore et al., 2011a; Morales et al., 2010b exploiting both BOMD and CEIMC (see fig. 14). The signature of the first order character of the transition is a plateau in $\mathrm{P}$ vs. $\rho$ along isotherms for temperature below $\approx 2000 \mathrm{~K}$. Both methods saw a density discontinuity at the transition of $\approx 2 \%$, sufficiently small that a quick scan of this region, as was done in previous simulations and experiments, could miss the discontinuity. However, the determined transition pressures with BOMD are $\approx 20 \%$ smaller than with CEIMC because to the band gap problem of local or semilocal density functionals. Inclusions of quantum proton effects within the DFT-GGA method, 
as obtained by PIMD, produce a further 25\% decrease of the transition pressure at the same temperature, because of the importance of nuclear zero point motion at the transition; the kinetic energy of the protons is higher in the molecular-rich phase than in the atomic-rich phase causing a shift in the transition pressure.

The DC electrical conductivity, as computed by the Kubo-Greenwood within DFT, undergoes a discontinuous jump across the transition, showing that it is an insulator-metal transition. Using the computed DC conductivity as order parameter, a critical point was estimated at $T_{c}=2000 \mathrm{~K}$. The transition corresponds to a sharp change from semiconducting to metallic behavior caused by the sudden collapse of the molecular state. Even though strong short range correlations persist after the transition is crossed, the resulting atomic liquid is metallic.

In figure 14 we show the LLPT from BOMD and CEIMC together with two different Kechin fits to the melting line data from BOMD for Phase I (see section IV.A.4). At low temperatures, the predicted LLT is expected to meet the melting line at $700 \mathrm{~K}$ and 220 $\mathrm{GPa}$ (using the BOMD estimates) and should result in a liquid-liquid-solid multiphase coexistence point. This point could be either a triple point if the two liquid phases coexist with a single solid phase, or a quadruple point if the metal-insulator transition extends below freezing. Unfortunately, the use of DFT to extend the melting curve to higher pressures has difficulties in the neighborhood of the insulator-metal transition. Moreover beyond $200 \mathrm{GPa}$ the stable crystal phase at melting is expected to be Phase IV, rather than Phase I; a study of the melting line for Phase IV has not been done. Also note that CEIMC simulations along the isotherm at $\mathrm{T}=600 \mathrm{~K}$ in the pressure interval $220 \mathrm{GPa} \leq P \leq 330 \mathrm{GPa}$ did not show any tendency to freeze, but finite size effects have biased the result. Further evidence in favor of a lower melting temperature at $P \simeq 300 G P a$ was given by an alternative QMC based ab-initio method (Attaccalite and Sorella, 2008) which observed a disordered state at $\approx 400 K$, shown in figure 14 as green star.

An independent determination(Lorenzen et al., 2010) of the LLPT line by FT-DFT-MD, shown in 14, gives general agreement, although the exact location of the LLPT line and critical point vary. The existence of the transition (continuous or first order) is sensitive to size effects since small systems at the $\Gamma$ point do not exhibit a discontinuous behavior. Morales et al., 2010b employed ground state DFT-GGA with 432 atoms at the $\Gamma$ point. Lorenzen et al., 2010 considered finite temperature DFT-GGA and systems of 512 atoms computed at the Baldereschi point in the Brillouin zone. These system sizes are comparable to the one used in (Scandolo, 2003) and (Bonev et al., 2004a) with CPMD, and considerably larger than the one used in (Vorberger et al., 2007b) and (Holst et al., 2008) with BOMD. Note that in CEIMC, a system of 54 atoms is used but with twist averaged boundary conditions; the many-body analog of the Brillouin zone integration.

In Bonev et al., 2004a the reentrant nature of the melting of the insulating molecular phase I reported in figure 14 was ascribed to an increased softening of the repulsive intermolecular interactions with pressure, more pronounced in the liquid than in the solid phase due to the presence of the disorder. The nature of the insulating molecular liquid near the melting line has been further investigated in Tamblyn and Bonev, 2010a,b. It was found that the liquid develops a short-range orientational order for pressures beyond the maximum of the melting line, and, in general, in the region between the melting and the LLPT lines. The orientational order is preserved, although changing its nature, when molecules dissociate. Using a criterium of survival time, the region of dissociation (from $67 \%$ to $33 \%$ molecular fraction), reported in figure 14 as a red-shaded area, has been traced up to $\mathrm{T}=4000 \mathrm{~K}$, close to the primary Hugoniot. Since pressure dissociation and metallization are seen to occur at the same time, the red shaded region also indicates metallization. This simulation is in agreement with some estimates of metallization obtained by laser-shocking a pre-compressed sample(Loubeyre et al., 2004) as shown in figure 14.

\section{Optical and Transport Properties}

Along with EOS studies, first-principles simulation methods have been used extensively to study the optical and transport properties of hydrogen at high pressure, particularly in the liquid state, (Collins et al., 1998c, 2001b; Holst et al., 2011, 2008; Lambert et al., 2011; Lin et al., 2009; Pfaffenzeller et al., 1995). Optical properties are most often calculated using Kubo-Greenwood formula within linear response theory (Greenwood, 1958; Kubo, 1957) and DFT. Diffusion, viscosity, and other transport properties can be calculated from the dynamical correlation functions of the ionic trajectories. The thermal conductivity in the liquid atomic regime is typically calculated with the Wiedemann-Franz law (Franz and Wiedemann, 1853). A detailed description of the formulation is given in Holst et al., 2011 along with its application to dense hydrogen, in particular the electrical and thermal conductivity, thermopower, and Lorentz number as a function of density for temperatures between 10,000 $\mathrm{K}$ and 50,000 K. Figure 15 shows the electrical conductivity, for various densities, as a function of temperature. These calculations were extended(Lambert et al., 2011) using Orbital-Free Molecular Dynamics as well as DFT to densities up to $160 \mathrm{~g} / \mathrm{cm}^{3}$ and temperatures up to $800 \mathrm{eV}(\approx 10,000,000 \mathrm{~K})$; well within the plasma phase. They compare their results with plasma models and discuss the implications of their results for the simulation of capsule implosions for Inertial Confinement Fusion (ICF) experiments.

As discussed previously semi-local functionals in DFT underestimate the band-gap, and, as a consequence over- 


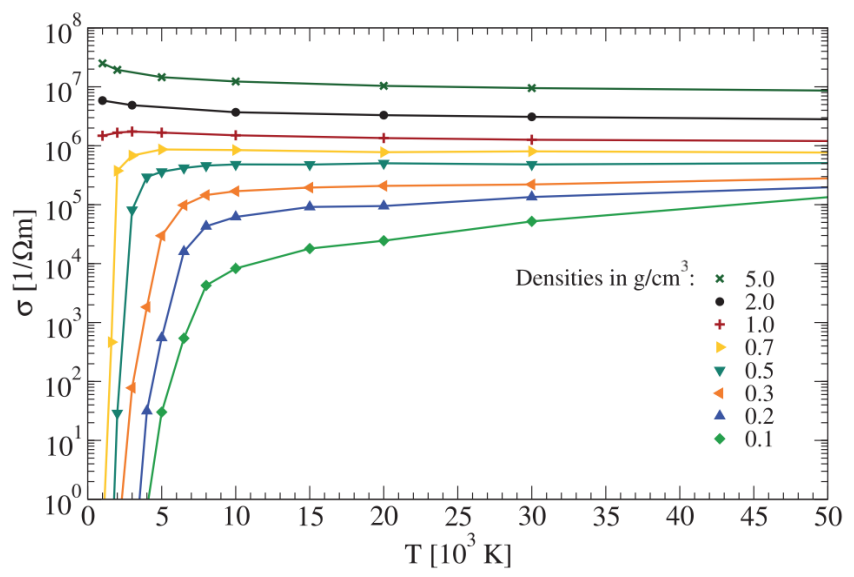

FIG. 15 Electrical conductivity of hydrogen as a function of temperature and density(Holst et al., 2011).

estimate the conductivity, particularly near the metalinsulator transition, i.e. close to the predicted liquidliquid phase transition or to the continuous dissociation region at higher temperatures. See (Kowalski et al., 2007) for a discussion of this error in dense helium. Calculations of the conductivity near dissociation have been performed (Lin et al., 2009) with QMC methods, using CEIMC simulations to sample ionic configurations at finite temperature, combined with correlation function quantum Monte Carlo calculations (Bernu et al., 1990; Ceperley and Bernu, 1988) to calculate the low energy many-body excitation spectrum of the liquid. Combining the excitation energies with the Green-Kubo formula, the authors calculated the electrical conductivity of hydrogen based entirely on QMC; these calculations do not suffer from self-interaction errors but suffer from other limitations, notably the numerical difficulty in obtaining accurate properties of excited states because of the QMC sign problem, and large finite cell size effects. However, good agreement with the limited data from shock experiments measurements was obtained (Weir et al., 1996).

\section{Quantum Phases of High Pressure Hydrogen}

Because of the light proton mass, it is possible that dense hydrogen could exhibit coherent quantum protonic phases at low temperature. Two interesting possibilities that have been theoretically predicted are superconductivity and superfluidity.

\section{Superconductivity in Solid Hydrogen}

Ashcroft, 1968 predicted that high-pressure hydrogen would be a high temperature superconductor. Within the framework of Bardeen-Cooper-Schrieffer (BCS) theory (Bardeen et al., 1957), three key arguments support this prediction: (i) the light proton mass causes the vi- brational energy scale of the phonons to be remarkably high, e.g. $k_{\mathrm{B}}\langle\omega\rangle \approx 2100 \mathrm{~K}$ near $500 \mathrm{GPa}$, where $\langle\omega\rangle$ is the average phonon frequency. The prefactor in the expression for the critical temperature $T_{c}$ (see below) is also large; (ii) since the electron-ion interaction is due to the bare Coulomb attraction, the electron-phonon coupling should be strong; and (iii) at high pressures, the electronic density of states at the Fermi surface should be large and the Coulomb repulsion between electrons should be relatively low, typical features of a high-density system.

Ever since this prediction of high- $T_{c}$ superconductivity, there have been several efforts to predict $T_{c}$. However, since $T_{c}$ is sensitive to the assumed crystal structure(Whitmore et al., 1979), the predictions have varied widely. We limit the discussion to the most recently proposed structures for the molecular and atomic phases, as discussed in Sections IV.A.1 and IV.A.6, the $\mathrm{Cmca}$, $I 4_{1} / a m d$, and $R-3 m$ structures; for a discussion of the older predictions, the reader is referred to the introduction of McMahon and Ceperley, 2011b.

This essential ideas from Ashcroft, 1968 are elucidated in McMillan's estimate for $T_{c}$ (McMillan, 1968), which including the correction by Dynes (Dynes, 1972) can be written as

$$
k_{\mathrm{B}} T_{c}=\frac{\langle\omega\rangle}{1.2} \exp \left[-\frac{1.04(1+\lambda)}{\lambda-\mu^{*}(1+0.62 \lambda)}\right],
$$

where $\lambda$ is the electron-phonon-induced interaction, and $\mu^{*}$ is the renormalized Coulomb repulsion. It is easy to see that if $\langle\omega\rangle$ and $\lambda$ are high while $\mu^{*}$ is low, then $T_{c}$ will be high as well. Within standard $a b$ initio methods, such as DFT, one can calculate $\langle\omega\rangle$ and $\lambda$ (Savrasov and Savrasov, 1996), and we can approximate $\mu^{*} \sim 0.1$ in the atomic phase of high-pressure hydrogen (Richardson and Ashcroft, 1997). Note that this latter approximation does not work within the molecular phase - see below. Important corrections to Eq. (49) need to be included (Carbotte, 1990) for strong-coupling superconductors using the Allen-Dynes equation (Allen and Dynes, 1975); see a recent re-parameterization for hydrogen (Szczȩs̀niak and Jarosik, 2009).

Recently, McMahon and Ceperley, 2011b, 2012 investigated superconductivity in the atomic $I 4_{1} /$ amd and $R$ $3 m$ structures from $500 \mathrm{GPa}$ to $3.5 \mathrm{TPa}$. Calculated values of $T_{c}$ from this work are shown in Fig. 16.

As can be seen, the $T_{c}$ are indeed remarkably high, consistent with the original predictions (Ashcroft, 1968). Near the molecular-to-atomic transition, i.e. $500 \mathrm{GPa}$, the critical superconducting temperature $T_{c} \sim 311 \mathrm{~K}$. With increasing pressure, $\lambda$ increases, and is responsible along with the increase in the phonon frequencies for the increase in $T_{c}$ to $\sim 360 \mathrm{~K}$ near $0.8-1 \mathrm{TPa}$. After the first atomic-atomic structural phase transformation (i.e., $I 4_{1} /$ amd $\rightarrow R$-3m), a large jump in $\lambda$ occurs due to the high phonon density of states at low frequencies, resulting in $T_{c}$ 's larger than $400 \mathrm{~K}$.

In the molecular metallic phase, the situation becomes 


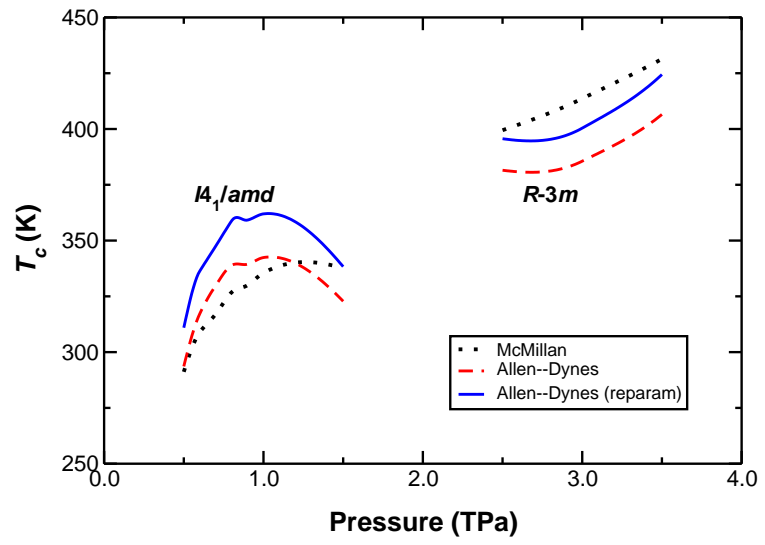

FIG. 16 (Color online) Values of $T_{c}$ for atomic metallic hydrogen calculated using various formulas McMahon and Ceperley, 2012, as discussed in the texts McMahon and Ceperley, 2011b, 2012.

especially interesting (but also more complex), as described by Richardson and Ashcroft, 1997. For example, the assumption that $\mu^{*}$ adopts a fixed value is invalid. Lüders et al., 2005 developed a multi-component DFT method for a superconducting state, termed SCDFT, which involves solving a set of Kohn-Sham equations for wave functions that represent particle and hole amplitudes, $\varphi_{i}(\boldsymbol{r})$, as well as protonic amplitudes, $\Phi_{l}(\boldsymbol{R})$,

$$
\begin{array}{r}
{\left[-\frac{\nabla^{2}}{2}+v_{\mathrm{s}}^{\mathrm{e}}[n, \chi, \Gamma](\boldsymbol{r})-\mu\right] \varphi_{i}(\boldsymbol{r})=\epsilon_{i} \varphi_{i}(\boldsymbol{r})} \\
{\left[-\sum_{\alpha} \frac{\nabla_{\alpha}^{2}}{2 M}+v_{\mathrm{s}}^{\mathrm{n}}(\boldsymbol{R})\right] \Phi_{l}(\boldsymbol{R})=\varepsilon_{l} \Phi_{l}(\boldsymbol{R})}
\end{array}
$$

where $n$ is the electronic density, $\chi$ is an "anomalous" density representing the order parameter characterizing a singlet superconducting state, $\Gamma$ is the diagonal part of the $N$-particle density matrix, $v_{\mathrm{s}}^{\mathrm{e}}(\boldsymbol{r})$ and $v_{\mathrm{s}}^{\mathrm{n}}(\boldsymbol{R})$ are Kohn-Sham potentials for the electrons and nuclei, and $\mu$ is the chemical potential. Following the development of this method, Cudazzo et al., 2008, 2010a,b modeled the high-pressure metallic molecular phase $\mathrm{Cmca}$. Calculated $T_{c}$ values are shown in Fig. 17, where it can be seen that, much like in the atomic phases, they are remarkably high, increasing up to $242 \mathrm{~K}$ near $450 \mathrm{GPa}$.

Thus, consistent with the original predictions (Ashcroft, 1968), $T_{c}$ values in hydrogen are remarkably high in both the molecular and atomic phases. The high values of $T_{c}$ combined with the low values of $T_{m}$ (Section IV.A.4) suggest the possibility that solid atomic hydrogen is always superconducting. There have also been predictions (Jaffe and Ashcroft, 1981) that liquid metallic hydrogen will also be superconducting above the melting temperature. From these results, and the discussion

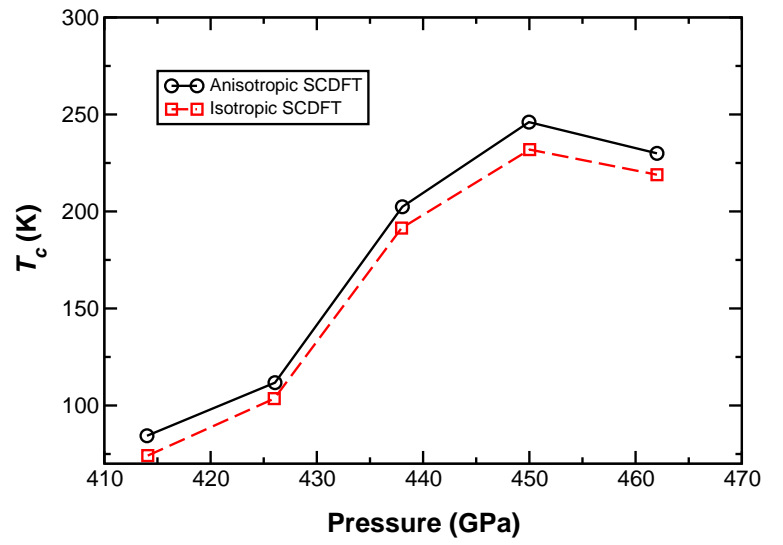

FIG. 17 (Color online) Values of $T_{c}$ for molecular hydrogen (the $C m c a$ phase) calculated using SCDFT (Cudazzo et al., 2010b).

that is to follow in the next Section IV.C.2), it can be seen that superconductivity in hydrogen is a rich area of exploration awaiting experimental confirmation.

\section{A Quantum Fluid?}

Brovman et al., 1972b noted that there are many metallic hydrogen structures with similar energies including anistropic layered structures. This suggests that the transition from the molecular state to the metallic state might also involve a transition from the solid phase to a low temperature liquid. There are two effects favoring the liquid metallic state. First, the bare proton-proton interaction is screened by the electrons, causing the effective interaction near $r_{s} \sim 1.3$ to be weak and shortranged. ${ }^{7}$ Friedel oscillations in the effective interaction can frustrate many crystal structures. Second, the large zero point motion of the protons favors a liquid protonic state versus an atomic crystal. In (Hohl et al., 1993) the kinetic energy of the protons in various crystal structures at zero temperature and at the lowest density of metallic hydrogen was estimated to be as large as $7000 \mathrm{~K}$ /atom. An analogous physical system is liquid ${ }^{3} \mathrm{He}$, which has a liquid ground state for pressures less than 34.4 bar.

Note that at sufficiently high density, it is almost certain that hydrogen will be in a liquid state at zero temperature. At very high density, we can ignore the electrons since they become uncorrelated with the protons. Hence, the proton system becomes the quantum one component plasma (OCP) i.e. jellium. Extensive QMC calculations (Ceperley and Alder, 1980) of the QOCP have estab-

\footnotetext{
7 At a certain density, electrons become bound to the protons and the interaction becomes one between neutral atoms.
} 
lished that the Wigner crystal melts to a Fermi liquid at $r_{s}=106$ using the electron mass or $r_{s}=0.058$ using the proton mass. This high pressure transition is indicated on Figure 2 at the extremely high pressure of $10^{13} \mathrm{GPa}$. However, it is unlikely that there are conditions anywhere where hydrogen is both this dense and this cold.

Simulations of the melting line of atomic hydrogen indicate that at somewhat lower densities (Kohanoff and Hansen, 1995, 1996; Xu et al., 1994) the atomic hydrogen lattice melts at surprisingly low temperatures. But note that these simulations were for classical protons in the bcc structure. More recent calculations by Liberatore et al., 2011b demonstrated that while the ZPE in atomic hydrogen is large, it has similar values in the solid and liquid phases because of similarities in local structures, and, thus, the overall impact of the proton motion on melting is not large.

The complete absence of an atomic solid phase was suggested by Kechin, 2004a,b (see again Section IV.A.4) due to the large atomic ZPM. However, many approximations were made in these calculations, in addition, to the assumption of the fcc structure and the use of the Lindemann criterion for melting. Furthermore, the results do not appear consistent with ab initio calculations (Kohanoff and Hansen, 1995, 1996; Liberatore et al., 2011b; $\mathrm{Xu}$ et al., 1994).

Going now back to the molecular phase, we saw in Section IV.A.4 that the molecular melting temperature reaches a maximum at a pressure of $100 \mathrm{GPa}$ and then decreases. Extrapolations of the atomic melting line from higher to lower pressures, combined with the decreasing melting line of molecular hydrogen (Bonev et al., 2004a), indicate the possibility of an intermediate range where a zero temperature liquid phase could exist (Ashcroft, 2000, 2003).

There was an early QMC calculation (Mon et al., $1980,1983)$ of the energy difference between the liquid and crystal phases. These calculations used an effective screened Coulomb interaction and found that the crystal state has lower energy than the liquid state for densities appropriate to the metallic hydrogen $0.8 \leq$ $r_{s} \leq 1.36$, partially based on the VMC calculations of (Ceperley et al., 1978) for the Yukawa phase diagram. Another DMC calculation(Ceperley, 1988) using the screened Coulomb interaction found crystal phases more stable for $r_{s} \leq 1.6$. Even if the crystal structures in these calculations were incorrect, the results seem to rule out the existence of an atomic ground state liquid. However, by far, the most serious approximation is the use of an effective proton interaction. Earlier work (Chakravarty and Ashcroft, 1978), showed that the assumption of a pair proton interaction in this range of density is likely to cause much greater errors than the difference between the liquid and crystal phase.

Turning now to direct simulation involving electrons and protons with the proper masses, Ceperley, 1988 using a diffusion Monte Carlo calculation of e-p system found stable fcc and bcc phases for densities above $r_{s}=1.6$, in the range of atomic hydrogen. Use of the more recent crystal structures (such as $I 4_{1} /$ amd) would further stabilize the crystal. However, since the fixed-node approximation and the time scale separation issue favor the crystal phase, the atomic liquid is still feasible. Supporting perturbation estimates(Chakravarty and Ashcroft, 1978), this DMC calculation found crystal-liquid energy differences with the bare interaction five times larger than with the screened coulomb interaction.

There was a recent classical MD simulation of hydrogen (Attaccalite and Sorella, 2008) of the physical system using forces computed from VMC with a RVB trial function. They found that a molecular liquid is more stable than a simple hexagonal or bcc atomic solid, both of which melted, at $400 \mathrm{~K}$ and 300GPA. Using this method, one needs to explore more stable crystal structures, different trial functions, put in quantum effects of the protons and a better treatment of finite size effects.

Liquid state effects combined with the predicted high superconducting critical temperatures in metallic hydrogen (Ashcroft, 1968) discussed above, led to a prediction (Jaffe and Ashcroft, 1981) that hydrogen could become a new state of matter altogether, a superconducting superfluid. Soon following, Jaffe and Ashcroft, 1983 analyzed the properties of such a state, and demonstrated that it would pass from a type-II to a type-I superconductor with decreasing temperature. Furthermore, at low temperatures, Ashcroft, 2000; Moulopoulos and Ashcroft, 1999 demonstrated that not only should electrons form Cooper pairs, but Cooper pairs of protons could also form. A topological analysis of this two-component system (Babaev et al., 2004)revealed that because of these features, in the presence of a magnetic field, hydrogen may exhibit several novel ordered states, ranging from metallic superfluids to superconducting-superfluids.

The predictions of a low-temperature liquid metallic state and its properties represent intriguing possibilities. Of course, the existence of such a state hinges crucially on the relative stabilities of the solid phases near the molecular-to-atomic transition, and given the recent predictions of crystal structures (see Sections IV.A.1 and IV.A.6), this is still an open question. Further experiments and AIMD, PIMD and CEIMC calculations are needed to settle this issue.

\section{HELIUM AND HYDROGEN-HELIUM MIXTURES}

Thermodynamic properties of hydrogen-helium mixtures have a special significance in the modeling of giant planets, particularly for Jupiter and Saturn. These planets, generally believed to have been formed approximately at the same time as the sun, are essentially made of hydrogen and helium, with a helium mass fraction at the surface of $\mathrm{Y}=0.234 \pm 0.005$ for Jupiter (von Zahn et al., 1998) and $\mathrm{Y}=0.18-0.25$ for Saturn (Conrath and Gautier, 2000). The estimated protosolar helium mass fraction is $\mathrm{Y} \sim 0.27$ (Bahcall et al., 1995). Possible inte- 


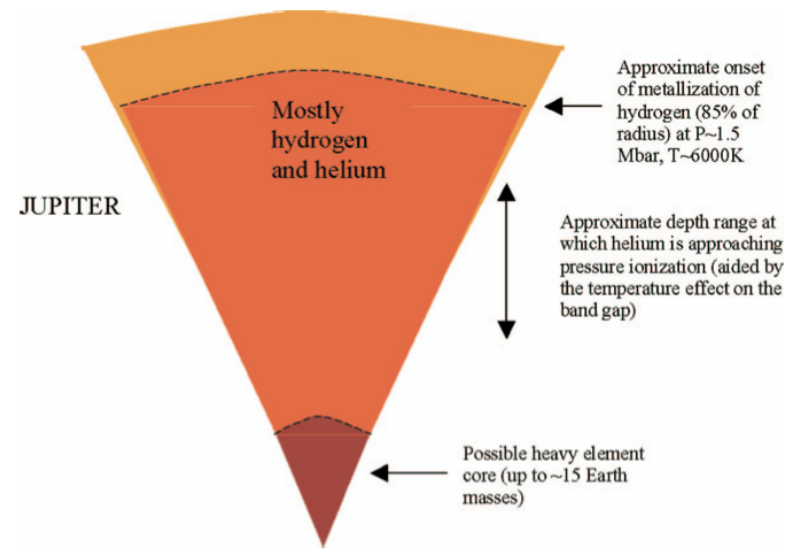

FIG. 18 (color online) Standard three layer model of Jupiter(Stevenson, 2008).

rior models for these planets, which define the composition of the planet as a function of depth, are constrained by experimental observations such as the total mass, radius, rotational rate, gravitational moments, surface temperatures, among others.

Most models built so far are based on a 3 layer structure with a solid core made of heavy elements and ices, an intermediate layer made up mostly of metallic hydrogen and helium, and an upper layer rich in molecular hydrogen, helium and small traces of other molecules like methane, water, and ammonia. See Fig. 18. The models typically assume solid body rotation, hydrostatic equilibrium, a fully convective and isentropic interior, and a homogeneous and constant mixture of helium in the metallic and molecular layers. These assumptions lead to a set of hydrostatic equations for the pressure, density, and entropy as a function of the planet's radius (Stevenson, 1982). In order to close the set of equations, the equation of state of the mixture is needed, specifically $P=P\left(\rho, T, x_{i}\right)$ where $x_{i}$ indicate the molar fractions of the mixture. Most current models of Jupiter and Saturn are based on the SCVH equations of state (Guillot, 1999; Guillot et al., 1994a,b, 1997), although recently, models for Jupiter have used equations of state derived from first-principles simulations (Militzer et al., 2008; Nettelmann et al., 2008). Currently, the largest uncertainty in the models comes from the limited knowledge of the equation of state of hydrogen-helium mixtures at high pressures and the uncertainty associated with possible transitions in the dense liquid: the Liquid-Liquid Phase Transition discussed above and the immiscibility of helium in metallic hydrogen (Fortney, 2004; Guillot, 2005) discussed below.

In this section, we review the current understanding of helium and hydrogen-helium mixtures based on firstprinciple calculations. In section V.A we review work on pure helium with emphasis on the phase diagram, the equation of state and optical properties at high pressure. In section V.B we discuss recent work on mixtures with emphasis on the calculation of the equation of state and the solubility of helium in metallic hydrogen.

\section{A. Helium}

At ambient conditions helium is an inert gas with a large band gap. Due to its low mass and weak interatomic interactions, it has fascinating properties at low temperatures and displays a wide array of exotic phenomena such as superfluidity. In this review, we focus only on the high pressure properties of the isotope ${ }^{4} \mathrm{He}$

\section{Equilibrium Properties: Equation of State and Structure}

At pressures above 25 bars $(2.5 \mathrm{MPa})$ at zero temperature, ${ }^{4} \mathrm{He}$ crystallizes into the hcp phase. Thermodynamic measurements show the existence of an hcp-fcc phase transition at $\mathrm{P} \sim 1.1 \mathrm{GPa}$, with a hcp-fcc-liquid triple point located approximately at $0.1 \mathrm{GPa}$ and 15 K (Dugdale and Simon, 1953). Early melting experiments using DAC techniques by Loubeyre et al., 1982, showed the existence of a cusp in the melting line at 11.65 GPa and $299 \mathrm{~K}$, suggesting the existence of a new phase at higher pressures. Single-crystal x-ray diffraction measurements at $300 \mathrm{~K}$ with DACs and synchrotron radiation showed that solid helium forms an hcp phase in the pressure range 15.6 to $23.3 \mathrm{GPa}$ (Mao et al., 1988); the stability of the hcp phase up to $58 \mathrm{GPa}$ was subsequently confirmed (Loubeyre et al., 1993). The melting line has been measured experimentally to pressures of 24 GPa by Vos et al., 1990, and up to 41 GPa by Datchi et al., 2000 with a resistively heated DAC. Recent measurements using laser-heated DACs extended the melting line to pressures up to $80 \mathrm{GPa}$ (Santamaria-Perez et al., 2010). There is good agreement in the measured melting lines up to $20 \mathrm{GPa}$, but the measurements of SantamariaPerez et al., 2010 are in disagreement with an extrapolation of the measurements of Datchi et al., 2000 to higher pressures. On the other hand, the extrapolation of the melting line of Vos et al., 1990 is in reasonable agreement with that of Santamaria-Perez et al., 2010. This disagreement has lead to some controversy regarding the high pressure phase diagram of helium. Classical MD simulations using empirical potentials have suggested the existence of a fcc-bcc phase transition above $12 \mathrm{GPa}$ (Koi et al., 2007), providing an alternative explanation to the measured cusp in the melting line. But since these results strongly depend on the empirical interatomic potential used to describe He, they do not resolve the discrepancy.

Various first-principles studies of the metallization transition in helium have been reported. The most recent calculations by Khairallah and Militzer, 2008 predict a zero temperature band gap closure at $25.7 \mathrm{TPa}$, using both QMC and GW methods. As expected, DFT calculations using semi-local functionals predict a transition pressure that is $40 \%$ smaller. On the other hand, perfect 
agreement is found between GW and QMC predictions. First-principles calculations of the elastic properties of solid helium were reported by (Nabi et al., 2005), showing a good agreement with experimental results (Zha et al., 2004).

Liquid helium at high pressures and temperatures has been the subject of numerous studies. The principal Hugoniot was measured using a two-stage light-gas gun,liquid helium at $4.3 \mathrm{~K}$ and 1 bar was shocked to 16 GPa and 12,000 $\mathrm{K}$ and double shocked to pressures of 56 GPa and 21,000 K (Nellis et al., 1984). The Hugoniot was recently extended to pressures over $100 \mathrm{GPa}$ by combining DACs and laser-driven shock wave techniques (Eggert et al., 2008b). Several pre-compressed states were shocked, enabling the exploration of an enlarged region of phase space.

Several chemical models for helium have been developed (Chen et al., 2007; Juranek et al., 2003; Saumon et al., 1995; Winisdoerfer and Chabrier, 2005), most of them built from perturbative expansions of the free energy using theoretical helium interatomic potentials, such as the Aziz potential (Aziz and Slaman, 1991) or the Ceperley-Partridge potential (Ceperley and Partridge, 1986). These models, e.g. SCVH, have been extensively used in planetary models of Jovian planets. At low pressures, a good agreement is found between experiments and these models. As pressure increases, electronic and thermal effects become very important and perturbative treatments become less accurate. Available experimental input is very limited.

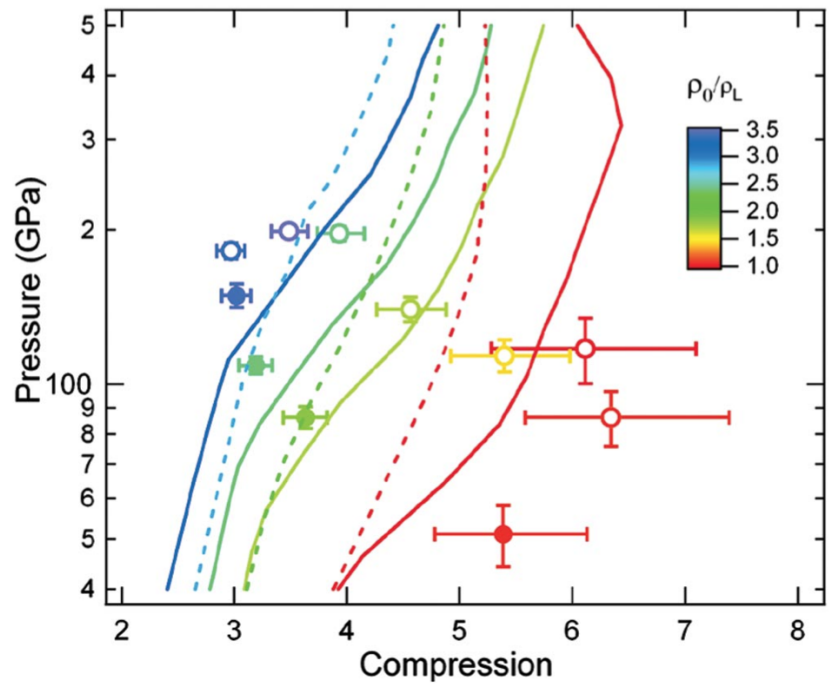

FIG. 19 (color online) Measurements of the principal helium Hugoniots for various initial densities. Symbols indicate the measured data, with open and closed symbols indicating whether the shocked state is reflecting or opaque, respectively. The solid line is the SCVH model (Saumon et al., 1995) and the dashed-line, ab initio calculations (Militzer, 2006). Figure taken from (Eggert et al., 2008b).

There are several first-principles studies of the thermo- dynamic properties of liquid helium (Kietzmann et al., 2007; Kowalski et al., 2007; Lorenzen et al., 2009; Militzer, 2005, 2006, 2009; Morales et al., 2009; Vorberger et al., 2007b), most of them using DFT-based MD with a semi-local functional, e.g. the PBE functional. Figure 19 shows a comparison of several Hugoniots of helium as measured and predicted by chemical models or firstprinciples simulations (Eggert et al., 2008b).

A recent study compared the EOS of helium between DFT-based BOMD and R-PIMC methods (Militzer, 2009); R-PIMC was used for temperatures above $60,000 \mathrm{~K}$ and DFT-MD for temperatures between $500 \mathrm{~K}$ and $125,000 \mathrm{~K}$, in the density range $0.38-5.35 \mathrm{~g} / \mathrm{cm}^{3}$. This work is particularly interesting because it establishes the importance of the electronic temperature in DFT-MD calculations and, in addition, compared predictions of both simulation methods for temperatures around 100,000 K, where both methods should be applicable and accurate. Good agreement between the methods was found for temperatures above 80,000 K, as long as the thermal occupation of the electrons is taken into account in DFT. These results suggest that the BornOppenheimer approximation is very good for calculating the equilibrium properties at temperatures below 100,000 K. Its influence on dynamical properties at such high temperatures, is still not well established.

At intermediate temperatures and pressures between $160 \mathrm{GPa}$ and $1600 \mathrm{GPa}$, the EOS has been studied in detail using the CEIMC method (Morales, 2009). Figure 20 shows the pressure of helium as a function of density as given by several chemical models: DFT-based BOMD and QMC-based CEIMC simulations. Good agreement exists between DFT and QMC-based simulation methods with these conditions. The chemical model, SCVH(Saumon et al., 1995), agrees reasonably well with first-principles simulation results, however, the WC model(Winisdoerfer and Chabrier, 2005) shows marked differences in magnitude and overall density dependence. This comparison is further evidence that DFT and QMC simulation methods agree well and thus make robust predictions of the equilibrium thermodynamic properties of helium at high pressure.

\section{Dynamic Properties: Optical and Transport Properties}

Advances over the last decade in the field of dynamic compression, particularly in their diagnostic tools, have enabled the direct measurement of optical properties of helium at pressures above $100 \mathrm{GPa}$ and temperatures up to 60,000 K (Celliers et al., 2010; Ternovoi et al., 2002). Figure 21 shows the measured reflectivity of helium as a function of temperature and density along several Hugoniots with different initial densities. The experiments suggest that helium undergoes an insulatorto-metal transition as either density or temperature are increased. The exact nature of the transition is still not clear, especially at low temperatures in the liquid. 


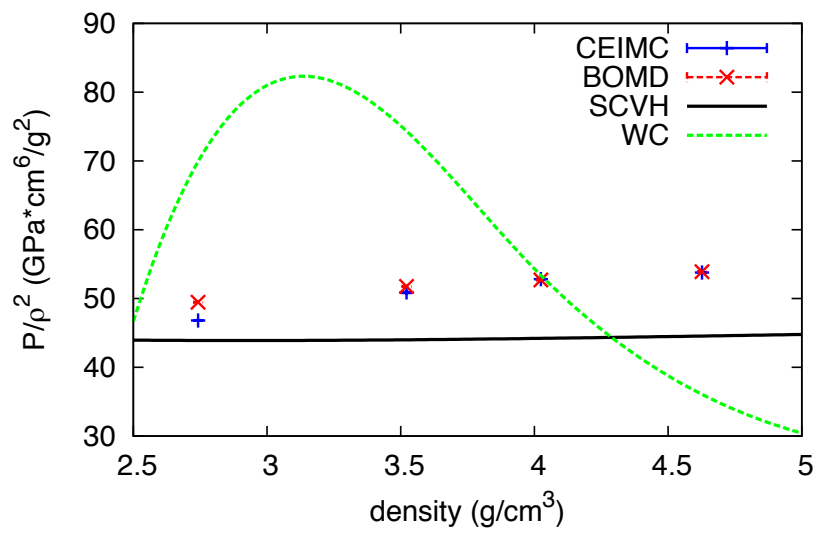

FIG. 20 (color inline)Comparison of the pressure of liquid helium as a function of density, along an isotherm at $\mathrm{T}=6000$ K, between CEIMC (Morales, 2009), DFT-based BOMD (Morales et al., 2009), and the SCVH (Saumon et al., 1995) and WC (Winisdoerfer and Chabrier, 2005) chemical models.

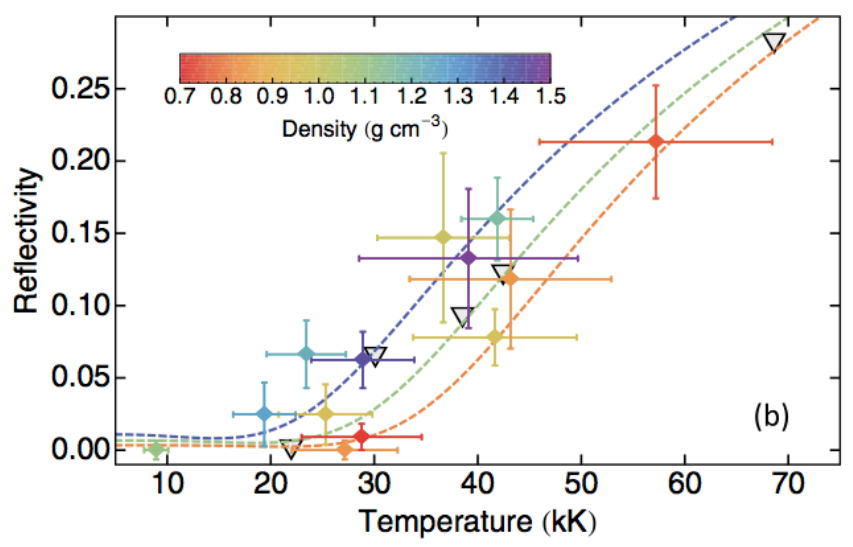

FIG. 21 (color online) Reflectivity measurements of helium using laser driven dynamic compression (Celliers et al., 2010). Solid diamonds show the observed reflectivity as a function of temperature and final density indicated by the color scale. Curves show the reflectivity obtained from a fit to the data using the semiconductor Drude model for three final state densities: $0.8,1.1$, and $1.4 \mathrm{~g} / \mathrm{cm}^{3}$. Gray triangles are calculated reflectivities by (Kowalski et al., 2007) near $1 \mathrm{~g} / \mathrm{cm}^{3}$ with a $+3 \mathrm{eV}$ gap correction. Figure and caption taken from (Celliers et al., 2010).

There have been various first-principles studies of the optical properties of liquid helium at high pressure (Hamel et al., 2011; Kietzmann et al., 2007; Kowalski et al., 2007; Lorenzen et al., 2011; Stixrude and Jeanloz, 2008). Using linear response theory within the KuboGreenwood formulation and DFT, Kowalski et al., 2007 calculated the band gap, conductivity, reflectivity, index of refraction, and absorption coefficient as a function of density and temperature. They studied the influence of the DFT exchange-correlation potential on the band gap of the liquid, clearly demonstrating the strong reduction in the gap due to self-interaction errors in semi-local functionals such as PBE. Using the GW method and hybridDFT calculations, they were able to devise a simple correction to the optical properties obtained from semi-local functionals, improving the agreement with experiment. They also showed that the self-interaction errors decrease with increasing temperature. Stixrude and Jeanloz, 2008 also calculated the band gap and the conductivity of liquid helium, extending to temperatures up to $50,000 \mathrm{~K}$ and densities up to $6 \mathrm{~g} / \mathrm{cm}^{3}$. Their work showed the gap closure occurs at much lower pressures in the liquid compared to the solid and depends strongly on temperature.

The first detailed study of the optical properties of hydrogen-helium mixtures using first-principles simulations came with the recent work of Hamel et al., 2011, where the conductivity and reflectivity of the mixture were calculated as a function of pressure, temperature and composition at pressures above $300 \mathrm{GPa}$ and temperatures above $4,000 \mathrm{~K}$. This corresponds to the region of pressure and temperature where helium becomes immiscible in metallic hydrogen (Morales et al., 2009). The authors use the frequency dependence of the reflectivity for both a mixed and a phase separated sample to predict a possible signature of phase separation in dynamic compression experiments. The conductivity and miscibility of hydrogen-helium mixtures across the dissociation regime has also been studied by Lorenzen et al., 2011 using first-principles methods.

\section{B. Phase separation of $\mathrm{H}$ and $\mathrm{He}$}

The EOS of hydrogen-helium mixtures is a key ingredient for the interior models of Jupiter, Saturn and other hydrogen-rich planets. In fact, the solubility of helium in hydrogen at high pressure plays a crucial role in the correct description of these planets. The giant planets typically radiate more energy than they take in from their sun. The current luminosity of Jupiter is well described with an evolution model for a convective homogeneous planet radiating energy left over from its formation 4.55 billion years ago. But a similar model for Saturn seriously underestimates its current luminosity (Hubbard et al., 1999). Although solar heating prolongs the cooling time of Saturn, its influence is not enough to reconcile its age with the expected time of formation. Hence, an additional energy source playing a more important role in Saturn than in Jupiter needs to be found.

Helium condensation has been proposed as a possible explanation for the excess luminosity in Saturn and the helium depletion in the atmosphere of both giant planets (Smoluchowski, 1967; Stevenson, 1975, 1979; Stevenson and Salpeter, 1977a,b). Let us suppose that there is a region in the planet's interior where helium is insoluble; helium droplets will form and thereby act as a source of energy, both through the release of latent heat, and by descending deeper into the center of the planet. Figure 22 shows a schematic representation of three differ- 


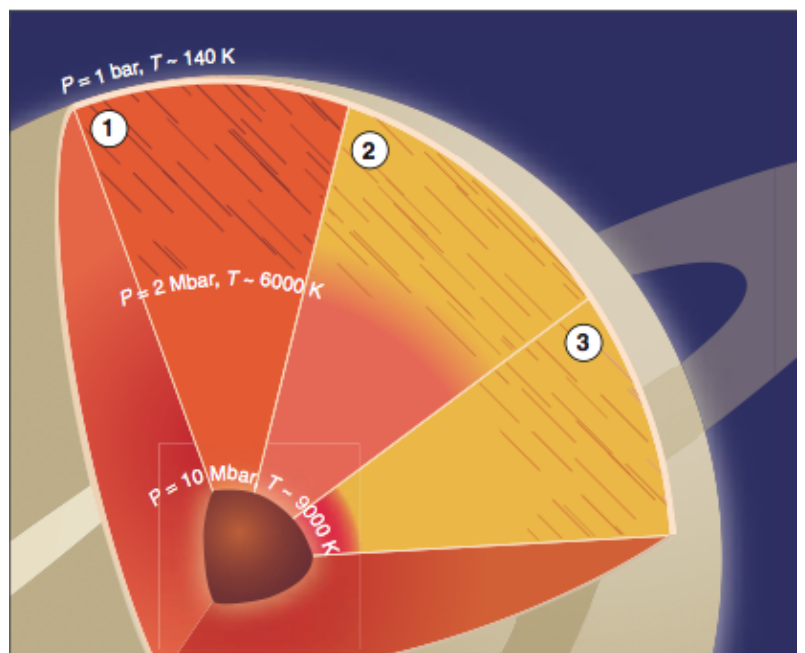

FIG. 22 (color online) Three views of the interior of Saturn. Orange represents the protosolar $\mathrm{He} / \mathrm{H}$ ratio. A yellower orange indicates less He, and a redder orange more He. Brown is the ice/rock core. The hashed regions indicate molecular hydrogen, while the unhashed regions atomic metallic hydrogen. (1) Saturn at an age of 1.5 billion years, before the onset of He phase separation. (2) The current Saturn according to a previously proposed H-He phase diagram (Stevenson, 1975). (3) The current Saturn according to a phase diagram derived from new evolutionary models (2). Figure and caption taken from (Fortney, 2004).

ent scenarios for the interior structure of Saturn based on possible mixing properties of helium in metallic hydrogen. Because Jupiter and Saturn have different total masses, the thermodynamic conditions in the planetary interiors could be such that this condensation process is more prevalent in Saturn than in Jupiter. Although this mechanism could explain most of the experimental observations in Saturn, an accurate understanding of the miscibility properties of helium on metallic hydrogen was only recently obtained using first-principles simulations.

This problem of helium solubility in metallic hydrogen has received great attention over the past 30 years. Before the development of first-principles simulations, calculations used perturbative treatments(Hansen et al., 1977; Hubbard and DeWitt, 1985; Pollock and Alder, 1977; Stevenson, 1979; Stevenson and Salpeter, 1977a,b; Straus et al., 1977) of the mixture free energy with assumed interactions between chemical species (e.g. He, $\mathrm{He}^{+}, \mathrm{H}, \mathrm{H}^{+}, \mathrm{H}_{2}$, etc.). Almost all calculations assumed a fully pressure-ionized mixture in order to facilitate the perturbative treatment. For the temperature and pressures relevant to planetary modeling, the assumption of complete ionization is now believed to be inaccurate, limiting the reliability of those calculations. Although the details of each calculation varied in terms of approach and complexity, most calculations found that the critical temperature for immiscibility decreased with increasing pressure and was generally too low to explain the observed discrepancy in the luminosity of Saturn (Fortney and Hubbard, 2004).

The first application of first-principles methods (Klepeis et al., 1991) used DFT calculations based on the local density approximation (LDA) to calculate the enthalpy of mixing of alloys of hydrogen and helium at zero temperature as a function of composition. Using the ideal mixing approximation, they obtained a demixing temperature of $15,000 \mathrm{~K}$ for $x_{H e}=0.07$, suggesting that there should be phase separation in both Jupiter and Saturn. However, their work neglected both the relaxation of the ionic crystal after the introduction of helium, and the disorder characteristic of a fluid. Using first-principles molecular dynamics (FPMD) simulations with the CarParrinello technique Pfaffenzeller et al., 1995 estimated the free energies of a mixture by a reweighting technique from the pure hydrogen liquid. They found a negligible temperature effect on the mixing free energy up to temperatures of $3,000 \mathrm{~K}$, disregarded thermal effects in the enthalpy of mixing, using instead the ideal mixing entropy. They obtained immiscibility temperatures too low to allow for differentiation in either Jupiter or Saturn.

The first proper ab-initio treatment of the problem came recently with the work of Morales et al., 2009. Using a combination of DFT-based BOMD calculations and thermodynamic integration, they calculated the Gibbs free energy of mixing as a function of pressure, temperature and composition without resorting to the ideal mixing approximation. Independent DFT-based BOMD calculations, by (Lorenzen et al., 2009), of the Gibbs free energy with the ideal mixing approximation for the entropy were also done. The two calculations agree very well in the regime where ideal mixing is valid.

To determine when the $\mathrm{H}-\mathrm{He}$ system is mixed, one needs the Gibbs free energy as a function of pressure, temperature and composition. The free energy is important, not only to calculate the critical concentrations, but also to produce accurate equations of state for planetary modeling. In the case of $\mathrm{H}-\mathrm{He}$ mixtures, the ideal mixing approximation significantly affects the resulting properties of planets and needs to be removed by direct calculation of the free energy.

Simulation methods such as Monte Carlo and Molecular Dynamics calculate ensemble averages of properties such as energy, pressure, density, etc. Free energies, or any property that directly involves the entropy, must be calculated by integrating along a thermodynamic pathway from a many-body system with known free energy (Frenkel and Smit, 2002). Until recently, this extra integration, in combination with the high computational cost of first-principles simulations, has prevented the evaluation of the Gibbs free energy of H-He mixtures. Morales et al., 2009 used a Coupling Constant Integration (CCI) to determine the free energy difference between a DFT description of the mixtures and that of a pair potential model tuned to produce a reasonable description of the DFT model. The pair potential simulation is many orders of magnitude faster than that of the the firstprinciple model and its free energy can be calculated by 


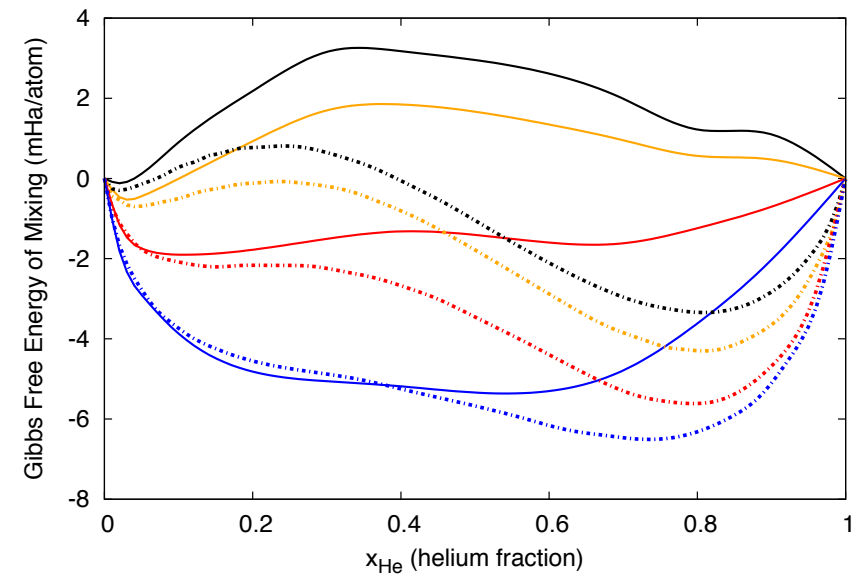

FIG. 23 The Gibbs free energy of mixing as a function of composition, at a pressure of $400 \mathrm{GPa}$, for several temperatures: $5000 \mathrm{~K}$ (black), $6000 \mathrm{~K}$ (orange), $8000 \mathrm{~K}$ (red) and $10000 \mathrm{~K}$ (blue). The solid lines represent full free energy calculations from Morales et al., 2009. The dashed lines represent results using the ideal mixing approximation for the entropy from Lorenzen et al., 2009.

integrating from the limit of zero density ${ }^{8}$

Using energies and pressures from ab-initio BOMD simulations on a grid of temperatures and densities Morales et al., 2009, calculated free energies for pressures between $300 \mathrm{GPa}$ and $1800 \mathrm{GPa}$, and temperatures between $4000 \mathrm{~K}$ and $10000 \mathrm{~K}$. In figure 23, the Gibbs free energy of mixing is shown as a function of composition, as calculated by Lorenzen et al., 2009, and Morales et al., 2009, at a pressure of $400 \mathrm{GPa}$. The strong temperature dependence of the immiscibility properties of the mixture are apparent from these results. A weak pressure dependence on the mixing free energies at low helium compositions is found. This leads to a weak dependence of critical mixing temperatures for compositions relevant to planetary interiors. Note how the Gibbs free energies reported by the different calculations agree well at low helium fractions, but disagree as the helium fraction increases.

As shown in Morales et al., 2009, the structure of hydrogen is strongly influenced by the helium concentration. The inert character of a helium atom makes it insensitive to changes in the local environment, however, a proton is very sensitive. While at low $\mathrm{x}_{H e}$, hydrogen is in the mono-atomic fully ionized state, an effective protonproton attraction reminiscent of the molecular bonding develops upon increasing $\mathrm{x}_{\mathrm{He}}$, even at very high pressures and temperatures. Figure 24 shows the proton-proton radial distribution functions for mixtures with various helium concentrations, at $8000 \mathrm{~K}$ for the density $r_{s}=1.05$.

\footnotetext{
${ }^{8}$ Care must be taken to ensure that first order phase transitions are not crossed during the integration.
}

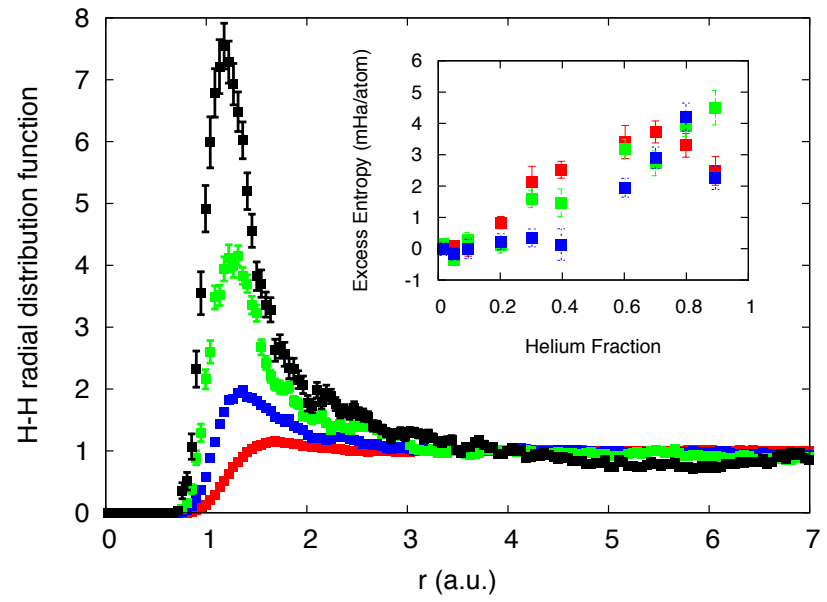

FIG. 24 The effect of increasing concentrations of helium on the proton-proton radial distribution function(Morales et al., 2009) for various compositions at a temperature of $8000 \mathrm{~K}$ and an electronic density given by $r_{s}=1.05: x_{H e}=0.9$ - black, $x_{H e}=0.8$ - green, $x_{H e}=0.6$ - blue, and $x_{H e}=0.0$ - red. . Inset: Excess entropic contribution of the Gibbs free energy of mixing, in $\mathrm{mHa} /$ atom, for several temperatures at a pressure of $800 \mathrm{GPa}: \mathrm{T}=6,000 \mathrm{~K}-\operatorname{red}, \mathrm{T}=8,000 \mathrm{~K}-$ green, and $\mathrm{T}=$ $10,000 \mathrm{~K}$ - blue.

A molecular-like peak builds up smoothly as $\mathrm{x}_{H e} \rightarrow 1$. The inert helium inhibits the delocalization of the hydrogenic electrons, enhancing the formation of weak molecular bonds with short lifetimes. A similar stabilization of molecular hydrogen by helium, but at much lower temperature and density, has been previously reported close to the dissociation regime in pure hydrogen by Vorberger et al., 2007b.

The change in the chemical properties of hydrogen produced by helium induces non-linear effects in the mixing functions. Figure 24 shows the excess non-ideal entropic contribution to the Helmholtz free energy, defined as: $S_{\text {excess }}(x)=S(x)+[x \ln (x)+(1-x) \ln (1-x)]$, at a pressure of $800 \mathrm{GPa}$. It is zero up to a helium fraction of $\mathrm{x}_{\mathrm{He}} \approx 0.2$, and increases after this point. As long as the fraction of helium is not large enough to induce the pseudo-molecular state in hydrogen, the ideal mixing approximation for the entropy is good. Once the pseudo-molecular state emerges, non-linear corrections to the mixing entropy appear and the full mixing free energy is needed to obtain an accurate description of the immiscibility process. The non-linear mixing entropy is roughly $3 \mathrm{mHa}$ /atom at high helium fraction, comparable to the mixing Gibbs free energy. Calculations ignoring the excess will produce accurate results for $\mathrm{x}_{\mathrm{He}}<0.2$, but non-physical stable mixtures for $\mathrm{x}_{H e}>0.5$; compare Fig. 23 with the inset in Fig. 24. The difference between results of Lorenzen et al., 2009 and Morales et al., 2009 is well explained by the presence of a minimum in Lorenzen's mixing Gibbs free energies at $\mathrm{x}_{\mathrm{He}} \sim 0.8$ leading to demixing temperatures about $700 \mathrm{~K}$ higher. Otherwise the agreement between the calculations is excellent. 


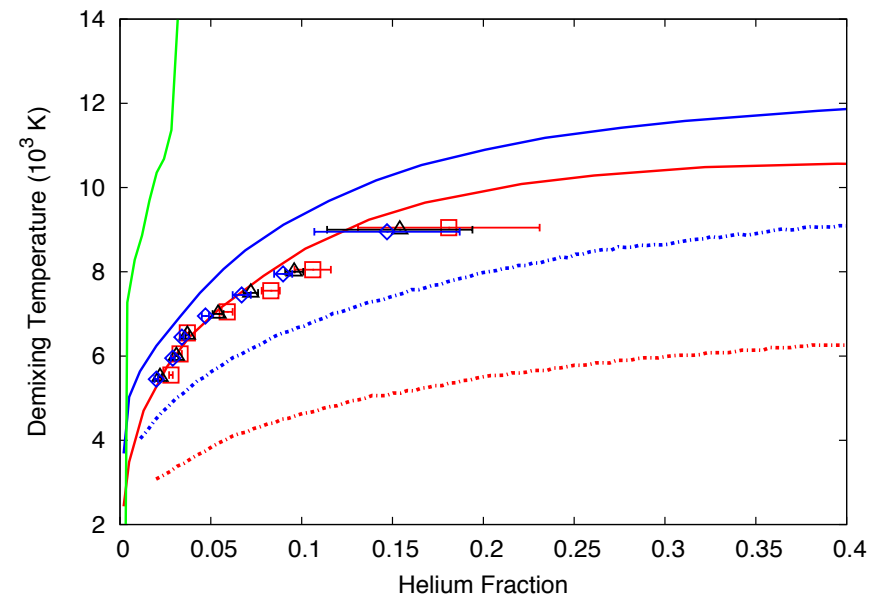

FIG. 25 (color online) Demixing temperatures as a function of the helium number fraction, for several pressures: $400 \mathrm{GPa}$ (red), 800 GPa (black), 1000 GPa (blue). Symbols represent results from Morales et al., 2009, solid lines are results from Lorenzen et al., 2009, dashed lines are results from Pfaffenzeller et al., 1995, and the green solid line is from Klepeis et al., 1991 at 1,050 GPa.

Figure 25 shows a comparison of demixing temperatures as a function of composition for several calculations described above. A prominent feature of the recent first-principles calculations is that pressure has only a moderate effect on the immiscibility process. For a fixed helium fraction, the demixing temperature changes by approximately $500 \mathrm{~K}$ in a pressure range of $800 \mathrm{GPa}$ for the relevant concentrations of $\mathrm{He}(5 \%$ to $10 \%)$. Immiscibility occurs at temperatures well below those required to produce ionization in helium (Stevenson, 2008); models with fully ionized He atoms are not appropriate for describing the pressure dependence of the demixing temperature. At pressures much higher than those discussed here, metallization of helium will play an important role and should produce significant changes to the pressure dependence of the immiscibility temperature presented here.

Recent estimates of the demixing temperature have important implications for the study of the interior structure of hydrogen-rich planets, especially Saturn. Firstprinciples results support the scenario that He becomes partially miscible in the intermediate layers of the planet, with the excess helium falling towards the core through gravitational differentiation. Whether the immiscible region is large enough to account for all the observed properties of Saturn is yet to be determined, but it is clear that planetary models should include phase separation. In general, the new DFT-based EOS could make a significant modification to interior models calculated with the SCVH EOS (Saumon et al., 1995). While models of Jupiter based on first principles EOS are beginning to appear (Militzer et al., 2008; Nettelmann et al., 2011), there is not yet a clear consensus on some of the details of the models. Nonetheless, the use of the new EOS is an important step forward which should help obtain more accurate models of planetary interiors.

\section{CONCLUSIONS AND OUTLOOK}

In this paper, we have summarized the current understanding of hydrogen and helium under extreme conditions. The last two decades have witnessed an enormous progress, both in experimental methodologies, and in simulation techniques for systems under extreme conditions. The synergy has greatly improved our understanding of high pressure hydrogen, helium and their mixtures. This review has focused on what has been learned from numerical simulation methods, much of which has only occurred very recently, now that such methods, computational techniques and computer hardware have advanced to the level of accuracy where important questions can be reliably answered.

The uncertainties in experimental findings are still too large; the experimentally achievable pressures and temperatures are still too limited. Experiments does not yet provide enough definitive information about dense hydrogen and helium. For example, DAC experiments at low temperatures have not been able to reach pressures where hydrogen conclusively becomes an atomic metal. Even though the recent experiment by Eremets and Troyan, 2011 claims to have achieved such a state, this interpretation is controversial (Jephcoat, 2011; Nellis et al., 2012), and the measurements are inconsistent with the semi-metallic behavior reported by Howie et al., 2012; Zha et al., 2012 under similar thermodynamic conditions. Although impressive advancements have been made and will come from new laser shock facilities, the results will continue to have large uncertainties. We nonetheless strongly encourage experiments to utilize such advances to verify the predictions that have already been made concerning the $L L T$ and $\mathrm{H}-\mathrm{He}$ demixing (see Sections IV.B.2 and V.B, respectively). Going forward, however, first principle simulations will continue to play a dominant and crucial role understanding and modeling materials at high pressure.

Some of the recent successes of simulations have been: a) the prediction of the reentrant nature of the melting line of the molecular hydrogen crystal, validated by several experimental measurements and theoretical confirmations; b) the prediction of a maximum compression of 4-4.5 along the principal Hugoniot of cryogenic $\mathrm{D}_{2}$, at variance with the first laser-induced shock experiments, but later confirmed by several other shock measurements; c) the prediction of a first-order phase transition line in the liquid phase of hydrogen, using both CEIMC and FPMD which has received some experimental support (see fig. 14) but still requires a systematic experimental validation; and $\mathrm{d}$ ) the prediction of the demixing temperature in $\mathrm{H}-\mathrm{He}$ mixtures which can account for the differences between the planets Jupiter and Saturn. A number of other predictions have been made but require 
experimental verification such as the crystal structures and superconductivity in solid atomic hydrogen.

As we have illustrated, away from the metallization and molecular dissociation region, FPMD methods are very accurate when compared to experiments and to more fundamental methods such as CEIMC and RPIMC. There are programs underway to make a complete survey of the phase diagram for all values of pressure temperature and composition (i.e., of hydrogen to helium) using both FPMD and RPIMC. The metallization region is of great relevance for planetary modeling, for ICF applications at high temperature and for fundamental physics understanding at low temperature,. For applications, quantitative details matter. Current FPMD, based on the GGA functional within the DFT, has errors as a result of the limitations of the GGA in treating excited states and band gaps. Quantitative predictions in this region require more accurate description of electronic correlation such as QMC or the use of more appropriate functionals in DFT. While application of QMC description of correlation in the CEIMC method has been applied, the improved functionals in FPMD have not been widely used in dense hydrogen and helium.

An important issue concerns the effects of the zero point motion of the nuclei. Quantum effects are likely to be important on the metallization transition below 1000 $\mathrm{K}$, in particular, since it occurs together with molecular dissociation. Zero point effects become more and more relevant at lower temperature, in particular in the molecular solid. The inclusion of these effects can change the relative stability of crystal phases (Natoli et al., 1993), are badly described by self-consistent harmonic approximations, but fully described using Path Integral methods. To achieve definitive calculations of superconductivity in dense hydrogen will likely require a complete treatment of the quantum mechanics of both the electrons and protons.

One of the major problems in past simulations has been lack of convergence: convergence in the number of particles, size of basis sets, number of reciprocal lattice vectors, length of time of dynamics, to mention the major issues. While it is true that the phases of hydrogen are very sensitive to the convergence issues, the systematic growth in power of the algorithms and computational power, means that the capability now exists to eliminate routinely such errors.

The fundamental bottleneck of QMC methods today is the "fermion sign problem" that was briefly discussed in section II.C.1. If the sign problem were solved, Quantum Monte Carlo methods could compute completely reliable solutions to the many-body quantum equations but, today, the exact methods are either severely limited in the number of electrons, or only give an upper bound to the internal energy. However progress can be made even without solving the sign problem. First, the sign problem is a scaling issue; it is possible to calculate exact properties of smaller systems (say dozens of atoms) and this number can be expected to increase in the future. These smaller system can act as benchmarks for the fixed-node QMC methods that scale to much larger sizes. Second, the variational fixed-node principle gives us an absolute signal when we have improved the trial function; substantial progress has been made in finding better trial functions in the past decade using this principle. Such an upper bound principle is lacking in DFT methods; instead they must rely on comparison with experiment or other methods. Third, the accumulated experience is that the fixed-node errors using existing trial functions are quite small for hydrogen and helium systems at high pressure, at least in the normal liquid and solid phases.

Despite a number of successes of simulation and experiment, many properties of hydrogen remain unresolved. Some of the most basic general questions that need answering concern the behavior of hydrogen at low temperature and increasing pressure. For example, a number of structures have been suggested for molecular phases II and III, and very recently for IV, but the agreement with experiment is not perfect. Furthermore, are there molecular phases at pressures beyond phases III and IV, and are they, in fact, those already predicted? At higher pressure, how does metallization occur, and what is the process of the molecular-to-atomic transition? Are these two effects related, or does metallization occur in the molecular phase via a band overlap? Are the recently proposed atomic structures via studies using the harmonic approximation for zero point motion correct? If not, how does the accurate treatment of proton ZPM affect their stabilities? Does it destabilize the lattice, giving a ground state quantum liquid? If not, at what pressure does the melting line reach a minimum, and at what temperature?

The use of the first principle simulation tools, in particular PIMD based on DFT, RPIMC and CEIMC will play pivotal roles in answering these questions. Most of the problems that held back progress can now be solved with known algorithms and existing computers. Simulations should be able to calculate the EOS to the accuracy needed for planetary modeling. Already EOS tables accurate to a few percent are available in certain regions of phase space. We anticipate in the next few years the computation and testing of these tables will be completed and accurate equilibrium properties for arbitrary $\mathrm{H}-\mathrm{He}$ mixtures will be available. Following that, advances in correlated methods are critically needed to compute more accurately properties such as electrical and thermal conductivity, viscosity, and opacity since these properties are also vital ingredients in planetary models and for other applications of matter in extreme conditions.

\section{Acknowledgments}

We acknowledge the interactions with many colleagues with whom we have worked on these problems with over the years including B. J. Alder, B. Bernu, K. Delaney, M. Dewing, M. Holzmann, E. Liberatore, Fei Lin, W. Magro, R. M. Martin, B. Militzer, V. Natoli, and E. 
Schwegler. The writing of this review was funded by the DOE grant DE-FG52-09NA29456, the Erasmus Mundus Scholarship, by the Materials Computation Center at the University of Illinois at Urbana-Champaign funded by NSF DMR-0325939. MAM is funded in part under the auspices of the US DOE by LLNL under Contract DEAC52-07NA27344. CP is supported by the Italian Institute of Technology (IIT) under the SEED project grant 259 SIMBEDD.

\section{References}

Abrikosov, A. A., 1954, Astron. Zh. 31, 112.

Akahama, Y., M. Nishimura, H. Kawamura, N. Hirao, Y. Ohishi, and K. Takemura, 2010, Phys. Rev. B 82, 060101(R).

Alavi, A., J. Kohanoff, M. Parrinello, and D. Frenkel, 1994, Phys. Rev. Lett. 73(19), 2599.

Allen, P. B., and M. Cardona, 1981a, Phys. Rev. B 24, 7479,

Allen, P. B., and M. Cardona, 1981b, Phys. Rev. B 23, 1495,

Allen, P. B., and R. C. Dynes, 1975, Phys. Rev. B 12, 905.

Ashcroft, N., and D. Stroud, 1978 (Academic Press), volume 33 of Solid State Physics, pp. $1-81$, .

Ashcroft, N. W., 1968, Phys. Rev. Lett. 21, 1748.

Ashcroft, N. W., 2000, J. Phys. Condens. Matter 12, A129.

Ashcroft, N. W., 2003, J. Phys. A - Math. Gen. 36, 6137.

Ashcroft, N. W., and N. D. Mermin, 1976, Solid State Physics (Holt, Rinehart and Winston).

Attaccalite, C., and S. Sorella, 2008, Phys. Rev. Lett. 100(11), 114501.

Aziz, R. A., and M. J. Slaman, 1991, 94(12), 8047, ISSN 00219606, .

Babaev, E., A. Sudbø, and N. W. Ashcroft, 2004, Nature 431, 666.

Baer, B. J., W. J. Evans, and C.-S. Yoo, 2007, Phys. Rev. Lett. 98, 235503.

Baer, B. J., W. J. Evans, and C.-S. Yoo, 2009, Phys. Rev. Lett. 102, 209901(E).

Bagnier, S., P. Blottiau, and J. Clérouin, 2000, Physical Review E 63(1), 015301.

Bahcall, J. N., M. H. Pinsonneault, and G. J. Wasserburg, 1995, Rev. Mod. Phys. 67, 781, .

Bailey, J., M. Knudson, A. Carlson, G. Dunham, M. Desjarlais, D. Hanson, and J. Asay, 2008, Physical Review B 78(14), 144107.

Bajdich, M., L. Mitas, G. Drobný, L. K. Wagner, and K. E. Schmidt, 2006, Phys. Rev. Lett. 96, 130201, .

Baraffe, I., G. Chabrier, and T. Barman, 2010, Reports on Progress in Physics 73(1), 016901,

Barbee III, T. W., and M. L. Cohen, 1991, Phys. Rev. B 44, 11563.

Barbee III, T. W., A. García, M. L. Cohen, and J. L. Martins, 1989, Phys. Rev. Lett. 62, 1150.

Bardeen, J., L. N. Cooper, and J. R. Schrieffer, 1957, Phys. Rev. 108, 1175.

Barker, J. A., 1979, The Journal of Chemical Physics 70(6), 2914,

Becke, A. D., 1993, J. Chem. Phys. 98, 5648.

Belov, S., G. Boriskov, A. Bykov, R. Il'Kaev, N. Luk'yanov, A. Matveev, O. Mikhailova, V. Selemir, G. Simakov, and
R. Trunin, 2002, JETP letters 76(7), 433.

Bernu, B., D. M. Ceperley, and W. A. Lester, 1990, J. Chem. Phys. 93, 552.

Beule, D., W. Ebeling, A. Förster, H. Juranek, R. Redmer, and G. Röpke, 1999, Contributions to Plasma Physics 39(12), 21.

Boehly, T., D. Hicks, P. Celliers, T. Collins, R. Earley, J. Eggert, D. Jacobs-Perkins, S. Moon, E. Vianello, and D. Meyerhofer, 2004, Physics of Plasmas 11, L49.

Bonev, S., E. Schwegler, T. Ogitsu, and G. Galli, 2004a, Nature 431(7009), 669.

Bonev, S. A., B. Militzer, and G. Galli, 2004b, Physical Review B 69(1), 014101.

Boriskov, G., A. Bykov, R. Il'Kaev, V. Selemir, G. Simakov, R. Trunin, V. Urlin, V. Fortov, and A. Shuikin, 2003, Doklady Physics 48(10), 553.

Boriskov, G., A. Bykov, R. Il'Kaev, V. Selemir, G. Simakov, R. Trunin, V. Urlin, A. Shuikin, and W. Nellis, 2005, Physical Review B 71(9), 092104.

Born, M., and J. Oppenheimer, 1927, Ann. Physik. 84, 457.

Brothers, E. N., A. F. Izmaylov, J. O. Normand, V. Barone, and G. E. Scuseria, 2008, The Journal of Chemical Physics 129(1), 011102 (pages 4), .

Brovman, E. G., Y. Kagan, and A. Kholas, 1972a, Sov. Phys. JETP-USSR 34, 1300.

Brovman, E. G., Y. M. Kagan, and A. Kholas, 1972b, Sov. Phys. JETP-USSR 35, 783.

Brush, S. G., H. L. Sahlin, and E. Teller, 1966, The Journal of Chemical Physics 45(6), 2102,

Burke, K., 2012, The Journal of Chemical Physics 136(15), 150901 (pages 9), .

Burrows, A., W. B. Hubbard, J. I. Lunine, and J. Liebert, 2001, Rev. Mod. Phys. 73, 719,

Caillabet, L., S. Mazevet, and P. Loubeyre, 2011, Physical Review B 83(9), 094101.

Cao, J., and B. J. Berne, 1993, The Journal of Chemical Physics 99(4), 2902,

Car, R., and M. Parrinello, 1985, Phys. Rev. Lett. 55(22), 2471.

Carbotte, J. P., 1990, Rev. Mod. Phys. 62, 1027,

Casula, M., C. Attaccalite, and S. Sorella, 2004, The Journal of Chemical Physics 121(15), 7110, .

Celliers, P., G. Collins, L. D. Silva, D. Gold, R. Cauble, R. Wallace, M. Foord, and B. Hammel, 2000, Phys. Rev. Lett. 84(24), 5564.

Celliers, P. M., P. Loubeyre, J. H. Eggert, S. Brygoo, R. S. McWilliams, D. G. Hicks, T. R. Boehly, R. Jeanloz, and G. W. Collins, 2010, Phys. Rev. Lett. 104, 184503,

Ceperley, D., M. Dewing, and C. Pierleoni, 2002, in Bridging Time Scales: Molecular Simulations for the Next Decade, edited by P. Nielaba, M. Mareschal, and G. Ciccotti (Springer-Verlag, Berlin), volume 605 of Topics in Condensed Matter Physics, p. 473.

Ceperley, D. M., 1988, Quantum Monte Carlo Simulation of Systems at High Pressure (Plenum).

Ceperley, D. M., 1992, Phys. Rev. Lett. 69, 331.

Ceperley, D. M., 1995, Rev. Mod. Phys. 67, 279.

Ceperley, D. M., 1996, in Monte Carlo and Molecular Dynamics of Condensed Matter Systems, edited by K. Binder and G. Ciccotti (Editrice Compositori, Bologna).

Ceperley, D. M., and B. J. Alder, 1980, Phys. Rev. Lett. 45, 566.

Ceperley, D. M., and B. J. Alder, 1981, Physica 108B, 875.

Ceperley, D. M., and B. J. Alder, 1987a, Phys. Rev. B 36, 
2092.

Ceperley, D. M., and B. J. Alder, 1987b, Phys. Rev. B 36, 2092.

Ceperley, D. M., and B. Bernu, 1988, J. Chem. Phys. 89, 6316.

Ceperley, D. M., G. V. Chester, and M. H. Kalos, 1977, Phys. Rev. B 16, 3081.

Ceperley, D. M., G. V. Chester, and M. H. Kalos, 1978, Phys. Rev. B 17, 1070.

Ceperley, D. M., and M. Dewing, 1999, J. Chem. Phys. 110, 9812.

Ceperley, D. M., and H. Partridge, 1986, J. Chem. Phys. 84, 820.

Ceriotti, M., D. E. Manolopoulos, and M. Parrinello, 2011, The Journal of Chemical Physics 134(8), 084104 (pages 9),

Ceriotti, M., M. Parrinello, T. E. Markland, and D. E. Manolopoulos, 2010, The Journal of Chemical Physics 133(12), 124104 (pages 13),

Chabrier, G., D. Saumon, and C. Winisdoerffer, 2007, Astrophysics Space Science 307, 263.

Chacham, H., and S. G. Louie, 1991, Phys. Rev. Lett. 66, 64,

Chacham, H., X. Zhu, and S. G. Louie, 1992, Phys. Rev. B 46, 6688,

Chakravarty, S., and N. W. Ashcroft, 1978, Phys. Rev. B 18, 4588.

Chen, Q., Y. Zhang, L. Cai, Y. Gu, and F. Jing, 2007, 14(1), 012703, ISSN 1070664X, .

Chiesa, S., D. M. Ceperley, R. M. Martin, and M. Holzmann, 2006, Phys. Rev. Letts. 97, cond-mat/0605004.

Clark, B., M. A. Morales, J. McMinis, J. Kim, and G. Scuseria, 2011, J. Chem. Phys 135,

Clouter, M., and H. P. Gush, 1965, Phys. Rev. Lett. 15, 200.

Collins, G., P. Celliers, L. D. Silva, R. Cauble, D. Gold, M. Foord, N. Holmes, B. Hammel, R. Wallace, and A. Ng, 2001a, Phys. Rev. Lett. 87(16), 165504.

Collins, G., L. D. Silva, P. Celliers, D. Gold, M. Foord, R. Wallace, A. Ng, S. Weber, K. Budil, and R. Cauble, 1998a, Science 281(5380), 1178.

Collins, G. W., P. Celliers, L. B. D. Silva, R. Cauble, D. Gold, M. Foord, K. S. Budil, R. Stewart, N. C. Holmes, M. Ross, B. A. Hammel, J. D. Kilkenny, et al., 1998b, Phys. Plasmas 5, 1864.

Collins, L., J. Kress, I. Kwon, W. Windl, T. Lenosky, N. Troullier, and R. Bauer, 1998c, Journal of ComputerAided Materials Design 5, 173, ISSN 0928-1045, 10.1023/A:1008605601624, .

Collins, L., I. Kwon, J. Kress, N. Troullier, and D. Lynch, 1995, Physical Review E 52(6), 6202.

Collins, L. A., S. R. Bickham, J. D. Kress, S. Mazevet, N. J. Troullier, and W. Windl, 2001b, Physical Review B 63(18), 184110.

Conrath, B. J., and D. Gautier, 2000, Icarus 144(1), 124 , ISSN 0019-1035,

Cudazzo, P., G. Profeta, A. Sanna, A. Floris, A. Continenza, S. Massidda, and E. K. U. Gross, 2008, Phys. Rev. Lett. 100, 257001.

Cudazzo, P., G. Profeta, A. Sanna, A. Floris, A. Continenza, S. Massidda, and E. K. U. Gross, 2010a, Phys. Rev. B 81, 134505.

Cudazzo, P., G. Profeta, A. Sanna, A. Floris, A. Continenza, S. Massidda, and E. K. U. Gross, 2010b, Phys. Rev. B 81, 134506 .
Cui, L., N. H. Chen, and I. F. Silvera, 1995, Phys. Rev. B 51 14987.

Cui, T., E. Cheng, B. J. Alder, and K. B. Whaley, 1997, Phys. Rev. B 55, 12253.

Da Silva, L. B., P. Celliers, G. W. Collins, K. S. Budil, N. C. Holmes, T. W. Barbee Jr., B. A. Hammel, J. D. Kilkenny, R. J. Wallace, M. Ross, R. Cauble, A. Ng, et al., 1997, Phys. Rev. Lett. 78, 483,

DaSilva, L., P. Celliers, G. Collins, K. Budil, N. Holmes, T. Barbie Jr, B. Hammel, J. Kilkenny, R. Wallace, M. Ross, and R. Cauble, 1997, Phys. Rev. Lett. 78(3), 483.

Datchi, F., P. Loubeyre, and R. LeToullec, 2000, Phys. Rev. B 61, 6535 .

De Raedt, H., and B. De Raedt, 1983, Phys. Rev. A 28, 3575,

Deemyad, S., and I. F. Silvera, 2008, Phys. Rev. Lett. 100, 155701 .

Delaney, K., C. Pierleoni, and D. Ceperley, 2006, Phys. Rev. Lett. 97(23), 235702.

Desjarlais, M., 2003, Physical Review B 68(6), 064204.

Dharma-wardana, M. W. C., and F. m. c. Perrot, 2002, Phys. Rev. B 66, 014110,

Diatschenko, V., and C. W. Chu, 1981, Science 212(4501), 1393,

Diatschenko, V., C. W. Chu, D. H. Liebenberg, D. A. Young, M. Ross, and R. L. Mills, 1985, Phys. Rev. B 32, 381.

Dick, R., and G. Kerley, 1980, J. Chem. Phys. 73, 5264.

Dion, M., H. Rydberg, E. Schröder, D. C. Langreth, and B. I. Lundqvist, 2004, Phys. Rev. Lett. 92, 246401, .

Driver, K., and B. Militzer, 2012, Phys. Rev. Letts. 108, 115502.

Drummond, N., R. J. Needs, A. Sorouri, and M. Foulkes, 2008, Phys Rev B 78, 125106.

Drummond, N. D., and R. J. Needs, 2005, Phys. Rev. B 72, 085124, .

Dugdale, J. S., and F. E. Simon, 1953, Proceedings of the Royal Society of London. Series A. Mathematical and Physical Sciences 218(1134), 291, .

Dykstra, C., 2005, Theory and applications of computational chemistry: the first forty years (Elsevier), ISBN 9780444517197, .

Dynes, R. C., 1972, Solid State Commun. 10, 615

Ebeling, W., and W. Richert, 1985, Phys. Status Solidi B 128(2), 467,

Ebeling, W., and R. W., 1985, Physics Letters A 108(2), 80,

Ebina, K., and H. Miyagi, 1989, Phys. Lett. A 142, 237.

Edwards, B., N. W. Ashcroft, and T. Lenosky, 1996, Europhys. Lett. 34, 519.

Edwards, P. P., M. T. J. Lodge, F. Hensel, and R. Redmer, 2010, Philosophical Transactions of the Royal Society A: Mathematical, Physical and Engineering Sciences 368(1914), 941, .

Eggert, J., S. Brygoo, P. Loubeyre, R. McWilliams, P. Celliers, D. Hicks, T. Boehly, R. Jeanloz, and G. Collins, 2008a, Phys. Rev. Lett. 100(12), 124503.

Eggert, J., S. Brygoo, P. Loubeyre, R. S. McWilliams, P. M. Celliers, D. G. Hicks, T. R. Boehly, R. Jeanloz, and G. W. Collins, 2008b, Phys. Rev. Lett. 100, 124503,

Ercolessi, F., and J. B. Adams, 1994, EPL (Europhysics Letters) $\mathbf{2 6}(8), 583$, .

Eremets, M., and I. Trojan, 2009, JETP Letters 89, 174, ISSN 0021-3640, 10.1134/S0021364009040031.

Eremets, M. I., and I. A. Troyan, 2011, Nat. Mater. 10, 927. 
Feller, W., 1968, An introduction to probability theory and its applications (Wiley, New York).

Felsteiner, J., 1965, Phys. Rev. Lett. 15, 1025.

Fermi, E., 1927, Rend. Accad. Naz. Lincei 6, 602.

Feynman, R., 1939, Phys. Rev. 56, 340.

Feynman, R., 1972, Statistical mechanics: a set of lectures, Frontiers in physics (W. A. Benjamin), .

Feynman, R., and A. Hibbs, 1965, Quantum Mechanics and Path Integrals (McGraw-Hill, New York, USA).

Filinov, V., M. Bonitz, P. Levashov, V. Fortov, W. Ebeling, M. Schlanges, and S. Koch, 2003, Journal of Physics A: Mathematical and General 36, 6069.

Filinov, V., P. Levashov, M. Bonitz, and V. Fortov, 2005, Plasma physics reports 31(8), 700.

Fiolhais, C., F. Nogueira, and M. Marques, 2003, A primer in density functional theory, Lecture notes in physics (Springer), ISBN 9783540030836, .

Fortney, J., 2004, Science 305, 1414.

Fortney, J., and N. Nettelmann, 2010, Space Science Reviews 152, 423, ISSN 0038-6308, 10.1007/s11214-009-9582-x, .

Fortney, J. J., and W. Hubbard, 2004, Astrophys J 608, 1039.

Fortney, J. J., and W. B. Hubbard, 2003, Icarus 164(1), 228 , ISSN 0019-1035, .

Fortov, V., V. Ternovoi, M. Zhernokletov, M. Mochalov, A. Mikhailov, A. Filimonov, A. Pyalling, V. Mintsev, V. Gryaznov, and I. Iosilevskii, 2003a, Journal of Experimental and Theoretical Physics 97(2), 259.

Fortov, V. E., R. I. Ilkaev, V. A. Arinin, V. V. Burtzev, V. A. Golubev, I. L. Iosilevskiy, V. V. Khrustalev, A. L. Mikhailov, M. A. Mochalov, V. Y. Ternovoi, and M. V. Zhernokletov, 2007, Phys. Rev. Lett. 99(18), 185001.

Fortov, V. E., V. Y. Ternovoi, M. V. Zhernokletov, M. A. Mochalov, A. L. Mikhailov, A. S. Filimonov, A. A. Pyalling, V. B. Mintsev, V. K. Gryaznov, and I. L. Iosilevskii, 2003b, JETP 97, 259.

Foulkes, W. M. C., L. Mitas, R. J. Needs, and G. Rajagopal, 2001, Rev. Mod. Phys. 73, 33.

Franz, R., and G. Wiedemann, 1853, Annalen der Physik 165(8), 497, ISSN 1521-3889, .

Freiman, Y. A., S. M. Tretyak, T. N. Antsygina, and R. J. Hemley, 2003, Journal of Low Temperature Physics 133, 251, ISSN 0022-2291, 10.1023/A:1026031107293,

Frenkel, D., and B. Smit, 2002, Academic Press. .

Friedli, C., and N. W. Ashcroft, 1977, Phys. Rev. B 16, 662,

Galam, S., and J.-P. Hansen, 1976, Phys. Rev. A 14, 816, .

Galli, G., R. Hood, A. Hazi, and F. Gygi, 2000, Physical Review B 61, 909.

Geneste, G., M. Torrent, F. Bottin, and P. Loubeyre, 2012, .

Giuliani, G., and G. Vignale, 2005, Quantum theory of the electron liquid (Cambridge University Press), ISBN 9780521821124, .

Goncharenko, I., and P. Loubeyre, 2005, Nature 435, 1206.

Goncharov, A. F., E. Gregoryanz, R. J. Hemley, and H. k. Mao, 2001, P. Natl. Acad. Sci. USA 98, 14234.

Goncharov, A. F., and R. J. Hemley, 2006, Chem. Soc. Rev. 35, 899.

Goncharov, A. F., R. J. Hemley, and H. k. Mao, 2011, J. Chem. Phys. 134, 174501.

Goncharov, A. F., R. J. Hemley, H. k. Mao, and J. Shu, 1998, Phys. Rev. Lett. 80, 101.

Goncharov, A. F., I. I. Mazin, J. H. Eggert, R. J. Hemley, and H. k. Mao, 1995, Phys. Rev. Lett. 75, 2514.

Greenwood, D. A., 1958, Proceedings of the Physical Society $\mathbf{7 1}(4), 585$, .

Gregoryanz, E., A. F. Goncharov, K. Matsuishi, H.-k. Mao, and R. J. Hemley, 2003, Phys. Rev. Lett. 90, 175701.

Grishechkin, S., S. Gruzdev, V. Gryaznov, M. Zhernokletov, R. Il'Kaev, I. Iosilevskii, G. Kashintseva, S. Kirshanov, S. Manachkin, and V. Mintsev, 2004a, JETP letters 80(6), 398.

Grishechkin, S., S. Gruzdev, V. Gryaznov, M. Zhernokletov, R. Ilkaev, I. Iosilevskii, G. Kashintseva, S. Kirshanov, S. Manachkin, V. Mintsev, A. Mikhailov, A. Mezhevov, et al., 2004b, JETP Letters 80, 398, ISSN 0021-3640, 10.1134/1.1830656, .

Guillot, T., 1999, Planetary and Space Science 47(10-11), 1183 , ISSN 0032-0633, .

Guillot, T., 2005, Annu. Rev. Earth Planet Sci. 33, 493.

Guillot, T., G. Chabrier, P. Morel, and D. Gautier, 1994a, Icarus 112(2), 354, ISSN 0019-1035, .

Guillot, T., D. Gautier, G. Chabrier, and B. Mosser, 1994b, Icarus 112(2), 337, ISSN 0019-1035,

Guillot, T., D. Gautier, and W. B. Hubbard, 1997, Icarus 130(2), 534 , ISSN 0019-1035, .

Guillot, T., and A. P. Showman, 2002, Astron. Astrophys. 385, 156.

Hafner, J., 2008, Journal of Computational Chemistry 29(13), 2044, ISSN 1096-987X, .

Hamel, S., M. A. Morales, and E. Schwegler, 2011, Phys. Rev. B 84, 165110, .

Hanfland, M., R. J. Hemley, and H.-k. Mao, 1993, Phys. Rev. Lett. 70, 3760, .

Hansen, J. P., 1973, Phys. Rev. A 8, 3096, .

Hansen, J. P., and I. R. McDonald, 1981, Phys. Rev. A 23, 2041 .

Hansen, J. P., I. R. McDonald, and E. L. Pollock, 1975, Phys. Rev. A 11, 1025, .

Hansen, J. P., G. M. Torrie, and P. Vieillefosse, 1977, Phys. Rev. A 16, 2153, .

Hawke, P., T. Burgess, D. Duerre, J. Huebel, R. Keeler, H. Klapper, and W. Wallace, 1978, Phys. Rev. Lett. 41(14), 994.

Hazen, R. M., H. K. Mao, L. W. Finger, and R. J. Hemley, 1987, Phys. Rev. B 36, 3944.

Hellmann, H., 1937, Franz Duetsche, Leipzig .

Hemley, R. J., and H. K. Mao, 1988, Phys. Rev. Lett. 61, 857.

Heyd, J., G. E. Scuseria, and M. Ernzerhof, 2003a, The Journal of Chemical Physics 118(18), 8207, .

Heyd, J., G. E. Scuseria, and M. Ernzerhof, 2003b, The Journal of Chemical Physics 118(18), 8207, .

Hicks, D., T. Boegly, P. Celliers, J. Eggert, S. Moon, D. Meyerhofer, and G. Collins, 2009, Physical Review B 79, 014112 .

Hohenberg, P., and W. Kohn, 1964, Phys. Rev. 136, B864.

Hohl, D., V. Natoli, D. M. Ceperley, and R. M. Martin, 1993, Phys. Rev. Lett. 71, 541.

Holmes, N., M. Ross, and W. Nellis, 1995, Physical Review B 52(22), 15835.

Holst, B., M. French, and R. Redmer, 2011, Phys. Rev. B 83, 235120, .

Holst, B., R. Redmer, and M. Desjarlais, 2008, Physical Review B 77(18), 184201.

Holzmann, M., B. Bernu, and D. M. Ceperley, 2011, Journal of Physics: Conference Series 321(1), 012020, .

Holzmann, M., D. M. Ceperley, C. Pierleoni, and K. Esler, 2003, Phys. Rev. E 68, 046707:1. 
Howie, R. T., C. L. Guillaume, T. Scheler, A. F. Goncharov, and E. Gregoryanz, 2012, Phys. Rev. Lett. 108, 125501,

$\mathrm{Hu}$, S., B. Militzer, V. Goncharov, and S. Skupsky, 2011, Phys. Rev. B 84, 224109.

Hubbard, W. B., 1972, Astrophys. J. 176, 525.

Hubbard, W. B., and H. E. DeWitt, 1985, Astrophys. J. 290, 388.

Hubbard, W. B., T. Guillot, M. S. Marley, A. Burrows, J. I. Lunine, and D. S. Saumon, 1999, Planet. Space Sci. 47, 1175.

Hubbard, W. B., and W. L. Slattery, 1971, Astrophys. J. 168, 131.

Huotari, S., J. A. Soininen, T. Pylkkänen, K. Hämäläinen, A. Issolah, A. Titov, J. McMinis, J. Kim, K. Esler, D. M. Ceperley, M. Holzmann, and V. Olevano, 2010, Phys. Rev. Lett. 105, 086403, .

Hutter, J., M. E. Tuckerman, and M. Parrinello, 1995, J. Chem. Phys. 102, 859.

Ichimaru, S., 1982, Rev. Mod. Phys. 54, 1017, .

Jaffe, J. E., and N. W. Ashcroft, 1981, Phys. Rev. B 23, 6176.

Jaffe, J. E., and N. W. Ashcroft, 1983, Phys. Rev. B 27, 5852.

Jeanloz, R., P. Celliers, G. Collins, J. Eggert, K. Lee, R. McWilliams, S. Brygoo, and P. Loubeyre, 2007, Proceedings of the National Academy of Sciences 104(22), 9172 .

Jephcoat, A. P., 2011, Nature Mater. 10, 904.

Johnson, K. A., and N. W. Ashcroft, 2000, Nature 403, 632.

Jones, M. D., and D. M. Ceperley, 1996, Phys. Rev. Lett. 76, 4572.

Jones, R. O., and O. Gunnarsson, 1989, Rev. Mod. Phys. 61, 689, .

Juranek, H., and R. Redmer, 2000, J. Chem. Phys. 112, 3780.

Juranek, H., V. Schwarz, and R. Redmer, 2003, Journal of Physics A: Mathematical and General 36, 6181.

Kagan, Y., V. V. Pushkarev, and A. Kholas, 1977, Sov. Phys. JETP-USSR 46, 511 .

Kaxiras, E., and J. Broughton, 1992, Europhys. Lett. 17, 151.

Kaxiras, E., J. Broughton, and R. J. Hemley, 1991, Phys. Rev. Lett. 67, 1138.

Kechin, V. V., 2004a, JETP Lett.+ 79, 40.

Kechin, V. V., 2004b, J. Phys. Condens. Matter 16, L125.

Keesom, W. H., J. de Smedt, and H. H. Mooy, 1930, Commun. Kamerlingh Onnes Lab., Univ. Leiden 19, 2090.

Kerley, G. I., 1972, Physics of the Earth and Planetary Interiors $6(1-3), 78$,

Kerley, G. I., 2003a.

Kerley, G. I., 2003b, unpublished

van Kessel, C. G. M., and R. Sigel, 1974, Phys. Rev. Lett. 33, 1020, .

Khairallah, S., J. Shumway, and E. Draeger, 2011, Arxiv preprint arXiv:1108.1711.

Khairallah, S. A., and B. Militzer, 2008, Phys. Rev. Lett. 101, 106407,

Kietzmann, A., B. Holst, R. Redmer, M. P. Desjarlais, and T. R. Mattsson, 2007, Phys. Rev. Lett. 98, 190602,

Kitaigorodskii, A. I., and K. V. Mirskaya, 1965, Sov. Phys. Crystallogr. 10, 121.

Kitamura, H., and S. Ichimaru, 1998, Journal of the Physics Society Japan 67(3), 950.

Kitamura, H., S. Tsuneyuki, T. Ogitsu, and T. Miyake, 2000, Nature 404, 259.

Klakow, D., C. Toepffer, and P. Reinhard, 1994a, J. Chem. Phys. 101, 10766.

Klakow, D., C. Toepffer, and P.-G. Reinhard, 1994b, Physics
Letters A 192(1), 55,

Klepeis, J. E., K. J. Schafer, T. W. Barbee, and M. Ross, 1991, Science 254, 986.

Knaup, M., P. Reinhard, C. Toepffer, and G. Zwicknagel, 2003, Journal of Physics A: Mathematical and General 36, 6165.

Knudson, M., and M. Desjarlais, 2009, Phys. Rev. Lett. 103(22), 225501.

Knudson, M., D. Hanson, J. Bailey, C. Hall, and J. Asay, 2003, Phys. Rev. Lett. 90(3), 35505.

Knudson, M., D. Hanson, J. Bailey, C. Hall, J. Asay, and W. Anderson, 2001, Phys. Rev. Lett. 87(22), 225501.

Knudson, M., D. Hanson, J. Bailey, C. Hall, J. Asay, and C. Deeney, 2004, Physical Review B 69(14), 144209.

Kohanoff, J., 2006, Electronic Structure Calculation for Solids and Molecules (Cambridge University Press).

Kohanoff, J., and J. Hansen, 1995, Phys. Rev. Lett. 74(5), 626.

Kohanoff, J., and J. Hansen, 1996, Phys. Rev. E 54(1), 768.

Kohanoff, J., S. Scandolo, G. L. Chiarotti, and E. Tosatti, 1997, Phys. Rev. Lett. 78, 2783.

Kohn, W., and L. Sham, 1965, Phys Rev 140(4A), 1133.

Kowalski, P. M., S. Mazevet, D. Saumon, and M. Challacombe, 2007, Phys. Rev. B 76, 075112,

Koi, L., R. Ahuja, A. B. Belonoshko, and B. Johansson, 2007, Journal of Physics: Condensed Matter 19(1), 016206, .

Kubo, R., 1957, J. Phys. Soc. Japan 12, 570.

Kümmel, S., and L. Kronik, 2008, Rev. Mod. Phys. 80, 3, .

Kwon, I., L. Collins, J. Kress, and N. Troullier, 1995, EPL (Europhysics Letters) 29, 537.

Kwon, I., L. Collins, J. Kress, N. Troullier, and D. Lynch, 1994a, Physical Review E 49(6), 4771.

Kwon, I., J. Kress, and L. Collins, 1994b, Physical Review B $\mathbf{5 0}(13), 9118$.

Kwon, Y., D. M. Ceperley, and R. M. Martin, 1993, Phys. Rev. B. 48, 12037.

Labet, V., P. Gonzalez-Morelos, R. Hoffmann, and N. W. Ashcroft, 2012a, The Journal of Chemical Physics 136(7), 074501 (pages 14),

Labet, V., R. Hoffmann, and N. W. Ashcroft, 2012b, The Journal of Chemical Physics 136(7), 074502 (pages 10), .

Labet, V., R. Hoffmann, and N. W. Ashcroft, 2012c, The Journal of Chemical Physics 136(7), 074503 (pages 10), .

Labet, V., R. Hoffmann, and N. W. Ashcroft, 2012d, The Journal of Chemical Physics 136(7), 074504 (pages 10), .

Lambert, F., V. Recoules, A. Decoster, J. Clerouin, and M. Desjarlais, 2011, Physics of Plasmas 18(5), 056306 (pages 9),

Landau, L., and G. Zeldovich, 1943, Acta Phys. Chim. USSR 18, 194.

Lee, K., E. D. Murray, L. Kong, B. I. Lundqvist, and D. C. Langreth, 2010, Phys. Rev. B 82, 081101, .

Lenosky, T., S. Bickham, J. Kress, and L. Collins, 2000, Physical Review B 61(1), 1.

Lenosky, T., J. Kress, and L. Collins, 1997a, Physical Review B 56(9), 5164.

Lenosky, T., J. Kress, L. Collins, and I. Kwon, 1997b, Physical Review B 55(18), 11907.

Lenosky, T., J. Kress, L. Collins, and I. Kwon, 1997c, Journal of Quantitative Spectroscopy and Radiative Transfer 58(4), 743.

Lenosky, T., J. Kress, L. Collins, R. Redmer, and H. Juranek, 1999, Physical Review E 60(2), 1665.

Liberatore, E., M. A. Morales, D. M. Ceperley, and C. Pier- 
leoni, 2011a, Molecular Physics , 1.

Liberatore, E., C. Pierleoni, and D. M. Ceperley, 2011b, J. Chem. Phys. 134(18), 184505.

Liebenberg, D. H., R. L. Mills, and J. C. Bronson, 1978, Phys. Rev. B 18, 4526, .

Lin, C., F.-H. Zong, and D. M. Ceperley, 2001, Phys. Rev. E 64, 016702.

Lin, F., M. A. Morales, K. T. Delaney, C. Pierleoni, R. M. Martin, and D. M. Ceperley, 2009, Phys. Rev. Lett. 103(25), 256401.

Lin, J.-F., M. Santoro, V. V. Struzhkin, H.-k. Mao, and R. J. Hemley, 2004, Rev. Sci. Instrum. 75, 3302.

Lindl, J. D., P. Amendt, R. L. Berger, S. G. Glendinning, S. H. Glenzer, S. W. Haan, R. L. Kauffman, O. L. Landen, and L. J. Suter, 2004, Physics of Plasmas 11(2), 339,

Liu, H., H. Wang, and Y. Ma, 2012, .

Lorenzana, H. E., I. F. Silvera, and K. A. Goettel, 1989, Phys. Rev. Lett. 63, 2080.

Lorenzana, H. E., I. F. Silvera, and K. A. Goettel, 1990, Phys. Rev. Lett. 64, 1939.

Lorenzen, W., B. Holst, and R. Redmer, 2009, Phys. Rev. Lett. 102, 115701.

Lorenzen, W., B. Holst, and R. Redmer, 2010, Physical Review B 82(19), 195107.

Lorenzen, W., B. Holst, and R. Redmer, 2011, Phys. Rev. B 84, 235109,

Loubeyre, P., J. M. Besson, J. P. Pinceaux, and J. P. Hansen, 1982, Phys. Rev. Lett. 49, 1172,

Loubeyre, P., P. Celliers, D. Hicks, E. Henry, A. Dewaele, J. Pasley, J. Eggert, M. Koenig, F. Occelli, and K. Lee, 2004, High Pressure Research 24(1), 25.

Loubeyre, P., R. LeToullec, J. P. Pinceaux, H. K. Mao, J. Hu, and R. J. Hemley, 1993, Phys. Rev. Lett. 71, 2272, .

Loubeyre, P., F. Occelli, and R. LeToullec, 2002, Nature 416, 613.

Lüders, M., M. A. L. Marques, N. N. Lathiotakis, A. Floris, G. Profeta, L. Fast, A. Continenza, S. Massidda, and E. K. U. Gross, 2005, Phys. Rev. B 72, 024545.

Magro, W., D. Ceperley, C. Pierleoni, and B. Bernu, 1996, Phys. Rev. Lett. 76(8), 1240.

Maksimov, E. G., and Y. I. Shilov, 1999, Physics-Uspekhi 42(11), 1121, .

Mao, H., and R. Hemley, 1989, Science 244, 1462.

Mao, H.-k., and R. J. Hemley, 1994, Rev. Mod. Phys. 66, 671.

Mao, H. K., R. J. Hemley, Y. Wu, A. P. Jephcoat, L. W. Finger, C. S. Zha, and W. A. Bassett, 1988, Phys. Rev. Lett. 60, 2649,

Marley, M. S., and W. B. Hubbard, 1988, Icarus 73(3), 536, .

Martin, R. M., 2004, Electronic Structure: Basic theory and practical methods (Cambridge University Press).

Martyna, G. J., D. J. Tobias, and M. L. Klein, 1994, J. Chem. Phys. 101, 4177.

Marx, D., and J. Hutter, 2000 (NIC, FZ, Jülich 2000), p. 301.

Marx, D., and J. Hutter, 2009, Ab initio molecular dynamics: Basic theory and advanced methods (Cambridge University Press).

Marx, D., and M. Parrinello, 1996, J. Chem. Phys. 104, 4077.

Matsuishi, K., E. Gregoryanz, H. Mao, and R. Hemley, 2003, J. Chem. Phys. 118, 10683.

Mattsson, A. E., P. A. Schultz, M. P. Desjarlais, T. R. Mattsson, and K. Leung, 2005, Modelling and Simulation in Materials Science and Engineering 13(1), R1,

Mazin, I. I., and R. E. Cohen, 1995, Phys. Rev. B 52, R8597,
McGrath, M. J., J. I. Siepmann, I.-F. W. Kuo, C. Mund, J. V. , J. H. ütter, F. Mohamed, and M. Krack, 2006, J. Phys. Chem. A 110, 646.

McMahon, J. M., and D. M. Ceperley, 2011a, Phys. Rev. Lett. 106, 165302.

McMahon, J. M., and D. M. Ceperley, 2011b, Phys. Rev. B 84, 144515, .

McMahon, J. M., and D. M. Ceperley, 2012, In Preparation . McMillan, W. L., 1965, Phys. Rev. 138, A442, .

McMillan, W. L., 1968, Phys. Rev. 167, 331.

Mermin, D., 1965, Phys. Rev. 137, A1441.

Metropolis, N., A. W. Rosenbluth, M. N. Rosenbluth, A. H. Teller, and E. Teller, 1953, J. Chem. Phys. 21, 1087.

Militzer, B., 2005, Journal of Low Temperature Physics 139, 739, ISSN 0022-2291.

Militzer, B., 2006, Phys. Rev. Lett. 97, 175501, .

Militzer, B., 2009, Phys. Rev. B 79, 155105, .

Militzer, B., and D. Ceperley, 2000, Phys. Rev. Lett. 85(9), 1890.

Militzer, B., and D. Ceperley, 2001, Phys. Rev. E 63(6), 066404

Militzer, B., D. Ceperley, J. Kress, J. Johnson, L. Collins, and S. Mazevet, 2001, Phys. Rev. Lett. 87(27), 275502.

Militzer, B., W. B. Hubbard, J. Vorberger, I. Tamblyn, and S. A. Bonev, 2008, Astrophys J Lett 45, 688.

Mills, R. L., and A. F. Schuch, 1965, Phys. Rev. Lett. 15, 722 .

Mills, R. L., J. L. Yarnell, and A. F. Schuch, 1973 (Plenum, New York, Boulder), volume 2, p. 202.

Mon, K. K., G. V. Chester, and N. W. Ashcroft, 1980, Phys. Rev. B 21, 2641.

Mon, K. K., G. V. Chester, and N. W. Ashcroft, 1983, Phys. Rev. B 27, 3895.

Moraldi, M., 2009, Phys. Rev. B 80, 134117.

Morales, M. A., 2009, First Principles Simulations of Hydrogen and Helium at High Pressure, Dissertation, University of Illinois at Urbana-Champaign.

Morales, M. A., L. X. Benedict, D. S. Clark, E. Schwegler, I. Tamblyn, S. A. Bonev, A. A. Correa, and S. W. Haan, 2012, High Energy Density Physics 8(1), 5 , ISSN 15741818

Morales, M. A., C. Pierleoni, and D. M. Ceperley, 2010a, Phys. Rev. E 81(2), 021202.

Morales, M. A., C. Pierleoni, E. Schwegler, and D. M. Ceperley, 2010b, Proceedings of the National Academy of Sciences 107(29), 12799, .

Morales, M. A., E. Schwegler, D. Ceperley, C. Pierleoni, S. Hamel, and K. Caspersen, 2009, Proc Natl Acad Sci USA 106, 1324.

Moshary, F., N. H. Chen, and I. F. Silvera, 1993, Phys. Rev. Lett. 71, 3814, .

Mostovych, A., Y. Chan, T. Lehecha, L. Phillips, A. Schmitt, and J. Sethian, 2001, Physics of Plasmas 8(15), 2281.

Mostovych, A., Y. Chan, T. Lehecha, A. Schmitt, and J. Sethian, 2000, Phys. Rev. Lett. 85(18), 3870.

Moulopoulos, K., and N. W. Ashcroft, 1999, Phys. Rev. B 59, 12309.

Nabi, Z., L. Vitos, B. Johansson, and R. Ahuja, 2005, Phys. Rev. B 72, 172102, .

Nagao, K., S. Bonev, B. A., and N. Ashcroft, 2003, Phys. Rev. Letts. 90, 35501.

Nagao, K., H. Nagara, and S. Matsubara, 1997, Phys. Rev. B 56, 2295.

Nagao, K., T. Takezawa, and H. Nagara, 1999, Phys. Rev. B 
59, 13741.

Nagara, H., 1989, J. Phys. Soc. Jpn. 58, 3861.

Nagara, H., and T. Nakamura, 1992, Phys. Rev. Lett. 68, 2468.

Nakamura, T., 1955, Prog. Theor. Phys. 14, 135.

Natoli, V., and D. M. Ceperley, 1995, J. Comput. Physics 117, 171 .

Natoli, V., R. M. Martin, and D. M. Ceperley, 1993, Phys. Rev. Lett. 70, 1952.

Natoli, V., R. M. Martin, and D. M. Ceperley, 1995, Phys. Rev. Lett. 74, 1601.

Nellis, W., 2006a, Reports on Progress in Physics 69, 1479.

Nellis, W., A. Louis, N. Ashcroft, R. Johnston, C. Rao, D. Tunstall, H. von Lohneysen, P. Edwards, and D. Logan, 1998, Philosophical Transactions: Mathematical, Physical and Engineering Sciences 356(1735), 119.

Nellis, W., A. Mitchell, P. McCandless, D. Erskine, and S. Weir, 1992, Phys. Rev. Lett. 68(19), 2937.

Nellis, W., A. Mitchell, M. V. Thiel, G. Devine, R. Trainor, and N. Brown, 1983a, J. Chem. Phys. 79, 1480.

Nellis, W., M. Ross, A. Mitchell, M. V. Thiel, D. Young, F. Ree, and R. Trainor, 1983b, Physical Review A 27(1), 608.

Nellis, W. J., 2006b, Rep. Prog. Phys. 69, 1479.

Nellis, W. J., N. C. Holmes, A. C. Mitchell, R. J. Trainor, G. K. Governo, M. Ross, and D. A. Young, 1984, Phys. Rev. Lett. 53, 1248,

Nellis, W. J., A. L. Ruoff, and I. F. Silvera, 2012, .

Nellis, W. J., S. T. Weir, and A. C. Mitchell, 1996, Science $\mathbf{2 7 3}(5277), 936$,

Nellis, W. J., S. T. Weir, and A. C. Mitchell, 1999, Phys. Rev. B 59, 3434,

Nettelmann, N., A. Becker, B. Holst, and R. Redmer, 2011, .

Nettelmann, N., B. Holst, A. Kietzmann, M. French, R. Redmer, and D. Blaschke, 2008, Astrophys. J. 683, 1217.

Norman, G. ., and A. N. Starostin, 1970, Journal of Applied Spectroscopy 13, 965, ISSN 0021-9037, 10.1007/BF00607515, .

Oliva, J., and N. W. Ashcroft, 1981a, Phys. Rev. B 24, 6200,

Oliva, J., and N. W. Ashcroft, 1981b, Phys. Rev. B 23, 6399,

Onida, G., L. Reining, and A. Rubio, 2002, Rev. Mod. Phys. $\mathbf{7 4}, 601$,

Overhauser, A. W., 1984, Phys. Rev. Lett. 53, 64.

Parr, R. G., and Y. Weitao, 1994, Density Functional Theory of Atoms and Molecules (Oxford University Press).

Payne, M. C., M. P. Teter, D. C. Allan, T. A. Arias, and J. D. Joannopoulos, 1992, Rev. Mod. Phys. 64, 1045, .

Perdew, J., K. Burke, and M. Enzerhof, 1996a, Phys. Rev. Lett. 77, 3865.

Perdew, J., and K. Schmidt, 2001 (American Institute of Physics), volume 577 .

Perdew, J., and A. Zunger, 1981, Phys. Rev. B 23, 5048.

Perdew, J. P., M. Ernzerhof, and K. Burke, 1996b, The Journal of Chemical Physics 105(22), 9982,

Perdew, J. P., and M. Levy, 1983, Phys. Rev. Lett. 51, 1884,

Pfaffenzeller, O., and D. Hohl, 1997, J. Phys.: Condens. Matter $\mathbf{9}, 11023$.

Pfaffenzeller, O., D. Hohl, and P. Ballone, 1995, Phys. Rev. Lett. 74(13), 2599.

Pickard, C. J., M. Martinez-Canales, and R. J. Needs, 2012, . Pickard, C. J., and R. J. Needs, 2006, Phys. Rev. Lett. 97,
045504.

Pickard, C. J., and R. J. Needs, 2007, Nature Phys. 3, 473.

Pierleoni, C., D. Ceperley, B. Bernu, and W. Magro, 1994, Phys. Rev. Lett. 73(16), 2145.

Pierleoni, C., and D. M. Ceperley, 2005a, ChemPhysChem 6(9), 1872, ISSN 1439-7641, .

Pierleoni, C., and D. M. Ceperley, 2005b, in Erice School proceedings, edited by G. Cicotti and K. Binder, physics/0510254.

Pierleoni, C., and D. M. Ceperley, 2006, Proceedings of the Internation School of Solid State Physics 34th course: Computer Simulations in Condensed Matter: from Materials to Chemical Biology, Lecture Notes in Physics 714, 641.

Pierleoni, C., D. M. Ceperley, and M. Holzmann, 2004, Phys. Rev. Letts. 93, 146402:1.

Pierleoni, C., K. Delaney, M. Morales, D. Ceperley, and M. Holzmann, 2008, Computer physics communications 179(1-3), 89.

Pierleoni, C., W.R.Magro, D. Ceperley, and B. Bernu, 1996, Physics of Strongly Coupled Plasmas, BINZ , 1.

Pollock, E. L., and B. J. Alder, 1977, Phys. Rev. A 15, 1263.

Pollock, E. L., and J. P. Hansen, 1973, Phys. Rev. A 8, 3110,

Raich, J. C., and H. M. James, 1966, Phys. Rev. Lett. 16, 173.

Ramaker, D. E., L. Kumar, and F. E. Harris, 1975, Phys. Rev. Lett. 34, 812,

Redmer, R., and B. Holst, 2010, in Metal-to-Nonmetal Transitions, edited by R. Redmer, F. Hensel, and B. Holst (Springer Berlin Heidelberg), volume 132 of Springer Series in Materials Science, ISBN 978-3-642-03953-9, pp. 63-84.

Richardson, C. F., and N. W. Ashcroft, 1997, Phys. Rev. Lett. 78, 118.

Robitaille, P.-M., 2011, Prog. Phys. 3, 60.

Ross, M., 1998, Physical Review B 58(2), 669.

Ross, M., F. Ree, and D. Young, 1983, J. Chem. Phys. 79, 1487.

Ross, M., and D. Seale, 1974, Phys. Rev. A 9, 396,

Rousseau, B., Y. Xie, Y. Ma, and A. Bergara, 2011, Eur. Phys. J. B 81, 1.

Runge, K. J., M. P. Surh, C. Mailhiot, and E. L. Pollock, 1992, Phys. Rev. Lett. 69, 3527.

Sano, T., N. Ozaki, T. Sakaiya, K. Shigemori, M. Ikoma, T. Kimura, K. Miyanishi, T. Endo, A. Shiroshita, and H. Takahashi, 2011a, Physical Review B 83(5), 054117.

Sano, T., N. Ozaki, T. Sakaiya, K. Shigemori, M. Ikoma, T. Kimura, K. Miyanishi, T. Endo, A. Shiroshita, H. Takahashi, T. Jitsui, Y. Hori, et al., 2011b, Phys. Rev. B 83, 054117,

Santamaria-Perez, D., G. D. Mukherjee, B. Schwager, and R. Boehler, 2010, Phys. Rev. B 81, 214101, .

Saumon, D., 2007, AIP Conf. Proc. 955, 101, .

Saumon, D., and G. Chabrier, 1989, Phys. Rev. Lett. 62(20), 2397, .

Saumon, D., and G. Chabrier, 1991, Physical Review A 44(8), 5122, .

Saumon, D., and G. Chabrier, 1992, Physical Review A 46(4), 2084 ,

Saumon, D., G. Chabrier, and H. V. Horn, 1995, The Astrophysical Journal Supplement Series 99, 713.

Saumon, D., and T. Guillot, 2004, The Astrophysical Journal $609(2), 1170$,

Savrasov, S. Y., and D. Y. Savrasov, 1996, Phys. Rev. B 54, 16487, . 
Scandolo, S., 2003, Proceedings of the National Academy of Sciences 100(6), 3051.

Sham, L. J., and M. Schlüter, 1983, Phys. Rev. Lett. 51, 1888,

Silvera, I., and V. Goldman, 1978, J. Chem. Phys. 69, 4209.

Silvera, I. F., 1980, Rev. Mod. Phys. 52, 393.

Silvera, I. F., and S. Deemyad, 2009, Low Temp. Phys.+ 35, 318.

Silvera, I. F., and R. J. Wijngaarden, 1981, Phys. Rev. Lett. 47, 39, .

Smoluchowski, R., 1967, Nature 215, 691.

Souvatzis, P., O. Eriksson, M. I. Katsnelson, and S. P. Rudin, 2008, Phys. Rev. Lett. 100, 095901, .

Städele, M., J. A. Majewski, P. Vogl, and A. Görling, 1997, Phys. Rev. Lett. 79, 2089, .

Städele, M., and R. M. Martin, 2000, Phys. Rev. Lett. 84, 6070.

Städele, M., M. Moukara, J. A. Majewski, P. Vogl, and A. Görling, 1999, Phys. Rev. B 59, 10031, .

Stevenson, D. J., 1975, Phys Rev B 12, 3999.

Stevenson, D. J., 1979, J Phys F Met Phys 9, 791.

Stevenson, D. J., 1982, Annu Rev Earth Planet Sci 10, 257.

Stevenson, D. J., 2008, Proc Natl Acad Sci USA 105, 11035.

Stevenson, D. J., 2010, (private communication) .

Stevenson, D. J., and E. E. Salpeter, 1977a, Astrophys J Suppl 35, 239.

Stevenson, D. J., and E. E. Salpeter, 1977b, Astrophys J Suppl 35, 221.

Stixrude, L., and R. Jeanloz, 2008, Proc Natl Acad Sci USA 105, 11071.

Straus, D. M., and N. W. Ashcroft, 1977, Phys. Rev. Lett. 38, 415.

Straus, D. M., N. W. Ashcroft, and H. Beck, 1977, Phys. Rev. B 15, 1914.

Subramanian, N., A. Goncharov, V. Struzhkin, M. Somayazulu, and R. Hemley, 2011, Proceedings of the National Academy of Sciences 108(15), 6014.

Surh, M. P., T. W. Barbee III, and C. Mailhiot, 1993, Phys. Rev. Lett. 70, 4090.

Surh, M. P., K. J. Runge, T. W. Barbee, E. L. Pollock, and C. Mailhiot, 1997, Phys. Rev. B 55, 11330.

Szczȩs̀niak, R., and M. W. Jarosik, 2009, Solid State Commun. 149, 2053.

Takahashi, M., and M. Imada, 1984, Journal of the Physical Society of Japan 53(11), 3765, .

Tamblyn, I., and S. Bonev, 2010a, Phys. Rev. Lett. 104(6), 65702.

Tamblyn, I., and S. A. Bonev, 2010b, J. Chem. Phys. 132(13), 134503.

Ternovoi, V. Y., A. S. Filimonov, A. A. Pyalling, V. B. Mintsev, and V. E. Fortov, 2002, AIP Conference Proceedings 620(1), 107, .

Theilhaber, J., 1992, Physics of Fluids B: Plasma Physics 4, 2044.

van Thiel, M., and B. J. Alder, 1966, Molecular Physics 10, 427 . .

van Thiel M., M. Ross, B. Hord, A. Mitchell, W. Gust, M. D'Addario, R. Keeler, and K. Boutwell, 1973, Phys. Rev. Lett. 31(16), 979.

Thomas, L. H., 1927, Proc. Cambridge Phil. Roy. Soc. 23, 542.

Tkatchenko, A., and M. Scheffler, 2009, Phys. Rev. Lett. 102, 073005 ,
Totsuji, H., and K. Tokami, 1984, Phys. Rev. A 30, 3175, .

Toulouse, J., and C. J. Umrigar, 2007, The Journal of Chemical Physics 126(8), 084102 (pages 16), .

Trotter, H., 1959, Proc. Am. Math, Soc. 10, 545.

Tuckerman, M. E., B. J. Berne, G. J. Martyna, and M. L. Klein, 1993, J. Chem. Phys. 99, 2796.

Tuckerman, M. E., and M. Parrinello, 1994a, J. Chem. Phys. 101, 1302.

Tuckerman, M. E., and M. Parrinello, 1994b, J. Chem. Phys. 101, 1316.

Umrigar, C. J., J. Toulouse, C. Filippi, S. Sorella, and R. G. Hennig, 2007, Phys. Rev. Lett. 98, 110201, .

Umrigar, C. J., K. G. Wilson, and J. W. Wilkins, 1988, Phys. Rev. Lett. 60, 1719,

Vieillefosse, P., and J. P. Hansen, 1975, Phys. Rev. A 12, 1106, .

Vorberger, J., I. Tamblyn, B. S. A, and B. Militzer, 2007a, Contributions to Plasma Physics 47(4-5), 375.

Vorberger, J., I. Tamblyn, B. Militzer, and S. Bonev, 2007b, Physical Review B 75(2), 024206.

Vos, W. L., M. G. E. van Hinsberg, and J. A. Schouten, 1990, Phys. Rev. B 42, 6106, .

Vuilleumier, R., 2006, in Computer Simulations in Condensed Matter Systems: From Materials to Chemical Biology Volume 1, edited by M. Ferrario, G. Ciccotti, and K. Binder (Springer Berlin / Heidelberg), volume 703 of Lecture Notes in Physics, ISBN 978-3-540-35270-9, pp. 223-285.

Wang, X. W., J. Zhu, S. G. Louie, and S. Fahy, 1990, Phys. Rev. Lett. 65, 2414,

Wang, Y., J. Lv, L. Zhu, and Y. Ma, 2010, Phys. Rev. B 82, 094116, .

Weir, S., 1998, J. Phys.: Condens. Matter 10, 11147.

Weir, S., A. Mitchell, and W. Nellis, 1996, Phys. Rev. Lett. 76(11), 1860.

Whitmore, M. D., J. P. Carbotte, and R. C. Shukla, 1979, Can. J. Phys. 57, 1185.

Wigner, E., and H. B. Huntington, 1935, J. Chem. Phys. 3, 1748.

Winisdoerfer, C., and G. Chabrier, 2005, Phys. Rev. E 71, 026402.

Woodley, S. M., and R. Catlow, 2008, NatMat 7, 937.

Xu, H., J.-P. Hansen, and D. Chandler, 1994, Europhys. Lett. 26, 419.

Yarnell, J. L., R. L. Mills, and A. F. Schuch, 1974, Sov. J. Low Temp. Phys. 1, 366.

von Zahn, U., H. D. M., and L. G., 1998, J. Geophys. Res. 103, 815.

Zeldovich, Y. B., and Y. P. Raizer, 1967, Physics of Shock Waves and High Temperature Phenomena (Academic Press, New York).

Zerah, G., J. Clerouin, and E. Pollock, 1992, Phys. Rev. Lett. 69(3), 446.

Zha, C.-S., Z. Liu, and R. J. Hemley, 2012, Phys. Rev. Lett. 108, 146402,

Zha, C.-S., H.-k. Mao, and R. J. Hemley, 2004, Phys. Rev. B 70, 174107, .

Zhang, L. J., Y. L. Niu, T. Cui, Y. Li, Y. M. Ma, Z. He, and G. T. Zou, 2007, J. Phys. Condens. Matter 19, 425237.

Zhang, L. J., Y. L. Niu, T. Cui, Y. Li, Y. Wang, Y. M. Ma, Z. He, and G. T. Zou, 2006, J. Phys. Condens. Matter 18, 9917. 